NASA/CR-2000-209415

ARL-CR-428
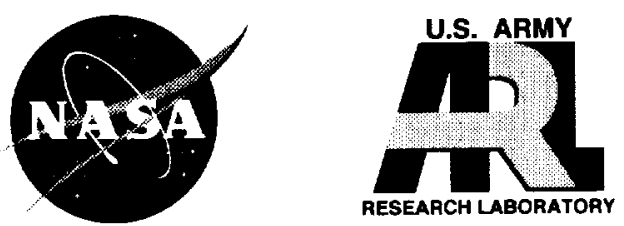

\title{
Computerized Generation and Simulation of Meshing and Contact of New Type of Novikov-Wildhaber Helical Gears
}

Faydor L. Litvin, Pin-Hao Feng, and Sergei A. Lagutin

The University of Illinois at Chicago, Chicago, Illinois 
Since its founding, NASA has been dedicated to the advancement of aeronautics and space science. The NASA Scientific and Technical Information (STI) Program Office plays a key part in helping NASA maintain this important role.

The NASA STI Program Office is operated by Langley Research Center, the Lead Center for NASA's scientific and technical information. The NASA STI Program Office provides access to the NASA STI Database, the largest collection of aeronautical and space science STI in the world. The Program Office is also NASA's institutional mechanism for disseminating the results of its research and development activities. These results are published by NASA in the NASA STI Report Series, which includes the following report types:

- TECHNICAL PUBLICATION. Reports of completed research or a major significant phase of research that present the results of NASA programs and include extensive data or theoretical analysis. Includes compilations of significant scientific and technical data and information deemed to be of continuing reference value. NASA's counterpart of peerreviewed formal professional papers but has less stringent limitations on manuscript length and extent of graphic presentations.

- TECHNICAL MEMORANDUM. Scientific and technical findings that are preliminary or of specialized interest, e.g., quick release reports, working papers, and bibliographies that contain minimal annotation. Does not contain extensive analysis.

- CONTRACTOR REPORT. Scientific and technical findings by NASA-sponsored contractors and grantees.
- CONFERENCE PUBLICATION. Collected papers from scientific and technical conferences, symposia, seminars, or other meetings sponsored or cosponsored by NASA.

- SPECIAL PUBLICATION. Scientific, technical, or historical information from NASA programs, projects, and missions, often concerned with subjects having substantial public interest.

- TECHNICAL TRANSLATION. Englishlanguage translations of foreign scientific and technical material pertinent to NASA's mission.

Specialized services that complement the STI Program Office's diverse offerings include creating custom thesauri, building customized data bases, organizing and publishing research results ... even providing videos.

For more information about the NASA STI Program Office, see the following:

- Access the NASA STI Program Home Page at http://www.sti.nasa.gov

- E-mail your question via the Internet to help@sti.nasa.gov

- Fax your question to the NASA Access Help Desk at 301-621-0134

- Telephone the NASA Access Help Desk at 301-621-0390

- Write to: NASA Access Help Desk NASA Center for AeroSpace Information 7121 Standard Drive Hanover, MD 21076 
NASA/CR-2000-209415
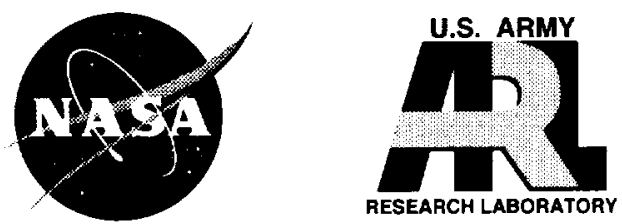

\section{Computerized Generation and Simulation of Meshing and Contact of New Type of Novikov-Wildhaber Helical Gears}

Faydor L. Litvin, Pin-Hao Feng, and Sergei A. Lagutin The University of Illinois at Chicago, Chicago, Illinois

Prepared under Contract NAS3-1992

National Aeronautics and Space Administration

Glenn Research Center 
Available from

NASA Center for Aerospace Information 7121 Standard Drive Hanover, MD 21076

Price Code: A04
National Technical Information Service 5285 Port Roval Road Springfield, VA 22100 Price Code: A04

Available electronically at http://gltrs.grc.nasa.gov/GLTRS 


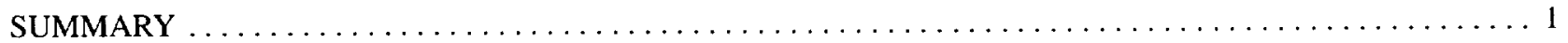

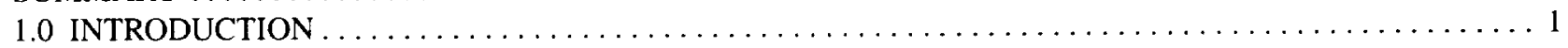

2.0 HELICAL PINION CONVEX AND GEAR CONCAVE PROFILE-CROWNED TOOTH SURFACES . . . . 7

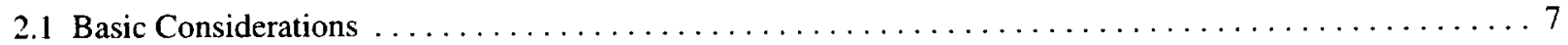

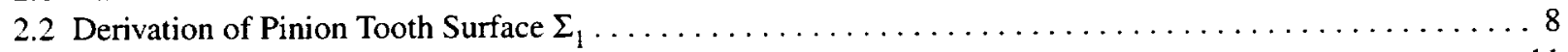

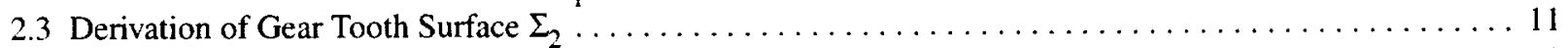

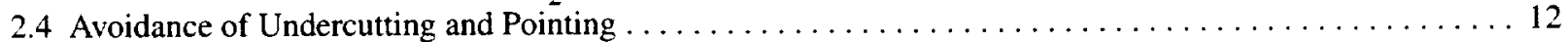

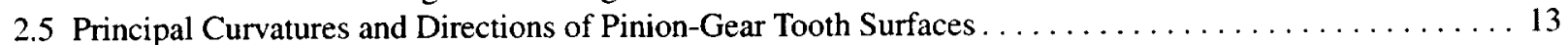

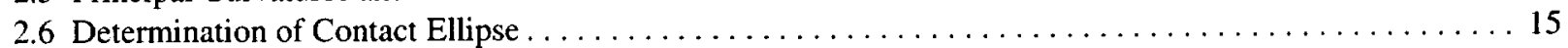

2.7 Recommended Parameters of Rack-Cutter Normal Profiles $\ldots \ldots \ldots \ldots \ldots \ldots \ldots \ldots$

2.8 Tooth Contact Analysis of Profile-Crowned Helical Gears $\ldots \ldots \ldots \ldots \ldots \ldots \ldots \ldots \ldots \ldots$

3.0 HELICAL PINION CONCAVE AND GEAR CONVEX PROFILE-CROWNED TOOTH SURFACES . . . . 20

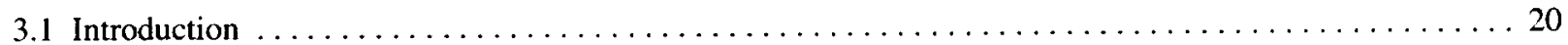

3.2 Preliminary Choice of Design Parameters of the Normal Section of Rack Cutters . . . . . . . . . . 20

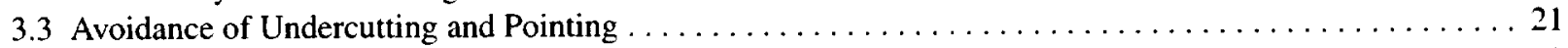

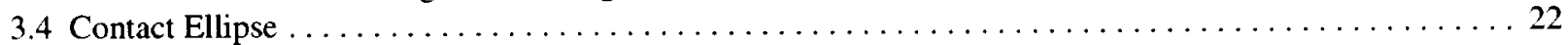

4.0 GENERATION OF DOUBLE-CROWNED PINION TOOTH SURFACE BY GRINDING DISK . . . . . 22

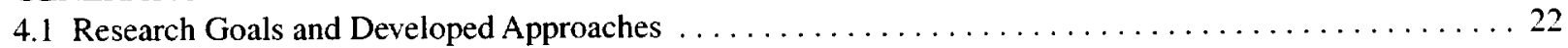

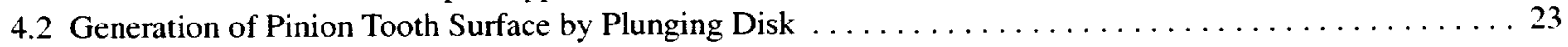

APPENDIX A-DETERMINATION OF SURFACE $\Sigma_{d}$ OF GENERATING DISK $\ldots \ldots \ldots \ldots$

APPENDIX B-COMPUTERIZED GENERATION OF PINION TOOTH SURFACE BY PLUNGING DISK . . . 28

5.0 GENERATION OF PINION TOOTH SURFACE BY PLUNGING OF GRINDING WORM . . . . . 31

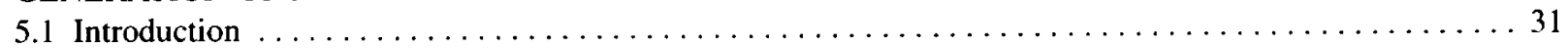

5.2 Installment, Related Motions, and Applied Coordinate Systems $\ldots \ldots \ldots \ldots \ldots \ldots$

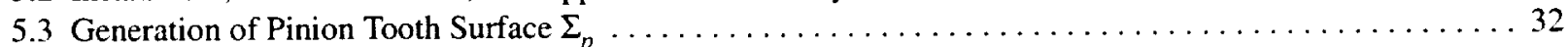

5.4 Relations Between Tool Plunging and Parabolic Function of Transmission Errors $\ldots \ldots \ldots \ldots$

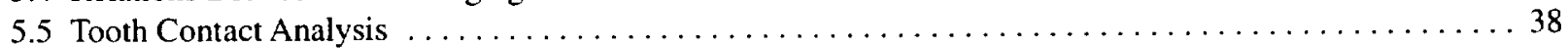

APPENDIX $\mathrm{C}-$ DERIVATION OF GRINDING WORM THREAD SURFACE $\ldots \ldots \ldots \ldots$

6.0 COMPARISON OF THREE TYPES OF NOVIKOV-WILDHABER HELICAL GEARS $\ldots \ldots \ldots \ldots$

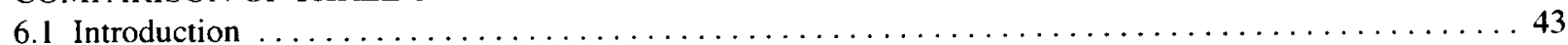

6.2 Description and Comparison of Three Types of Novikov-Wildhaber Gears $\ldots \ldots \ldots \ldots \ldots$

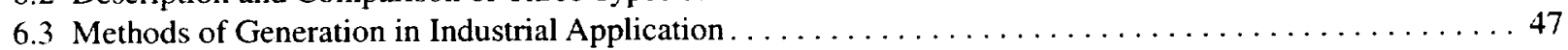

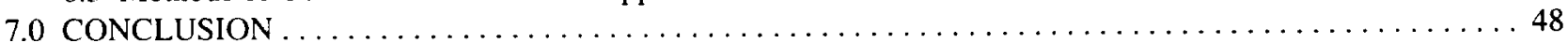

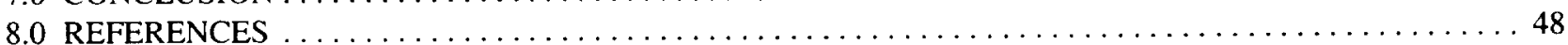





\title{
COMPUTERIZED GENERATION AND SIMULATION OF MESHING AND CONTACT OF NEW TYPE OF NOVIKOV-WILDHABER HELICAL GEARS
}

\author{
Faydor L. Litvin, Pin-Hao Feng, and Sergei A. Lagutin \\ The University of Illinois at Chicago \\ Chicago, Illinois 60607
}

\section{SUMMARY}

In this report, we propose a new geometry for low-noise, increased-strength helical gears of the NovikovWildhaber type. Contact stresses are reduced as a result of their convex-concave gear tooth surfaces. The gear tooth surfaces are crowned in the profile direction to localize bearing contact and in the longitudinal direction to obtain a parabolic function of transmission errors. Such a function results in the reduction of noise and vibrations.

Methods for the generation of the proposed gear tooth surfaces by grinding and hobbing are considered, and a tooth contact analysis (TCA) computer program to simulate meshing and contact is applied. The report also investigates the influence of misalignment on transmission errors and shift of bearing contact. Numerical examples to illustrate the developed approaches are proposed.

The proposed geometry was patented by Ford/UIC (Serial Number 09-340-824, pending) on June 28, 1999.

\subsection{INTRODUCTION}

In comparison with conventional involute helical gears, Novikov-Wildhaber (N-W) gears (refs. 1 and 2) have reduced contact stresses. This reduction has been achieved because at the point of contact, there is only a small difference between the curvatures of the concave and convex mating gear tooth surfaces. At every instant, the tooth surfaces are in a point contact spread under the load over an elliptical area; therefore, we say that the bearing contact is localized. There is a probability that the lubrication conditions of $\mathrm{N}-\mathrm{W}$ gears are slightly improved because in the process of meshing, the instantaneous contact ellipse moves not across the tooth surface but in the longitudinal direction. This direction of the contact path is favorable because it causes a "pumping effect." However, the same direction of the contact path can also be achieved for involute helical gears if profile modification is provided.

Novikov-Wildhaber gears or the generating imaginary rack-cutters are provided with circular-arc profiles. There are two versions of Novikov gears, the first having one zone of meshing (fig. 1.1) and the other having two. The design of gears with two zones of meshing was an attempt to reduce high bending stresses caused by point contact. Novikov gears with two zones of meshing have been standardized in the former USSR and in China (refs. 3 and 4). Figure 1.2 shows imaginary rack-cutters for the generation of Novikov gears with two zones of meshing.

Unfortunately for Novikov followers, who enthusiastically thought that N-W gears would be a substitute for conventional involute helical gears, their expectations have not been realized. After more than 40 years since their invention, N-W gears have found application only in low-speed reducers manufactured in the oil and mine industries. These gears are still applied in areas where manufacturers try to avoid grinding and expect that a sufficient bearing contact will be obtained by running under the load and lapping in the gear drive housing. Only soft materials are still applied for N-W gears, and the gear tooth surfaces are not hardened and nitrified.

The major disadvantage of $\mathrm{N}-\mathrm{W}$ gears is that gear misalignment (such as a change in the shaft angle or in the lead) causes an impermissible noise. Because of this high-level noise, the only example of the application of N-W gears in high-speed transmissions is that of the Westland Helicopter Company. The other disadvantage of N-W gears is the shift in the bearing contact (to the addendum or dedendum areas) that results from a change in the center distance. This shift can be reduced by increasing the difference between the curvatures of the tooth surfaces but this negates the attempt to reduce the contact stresses.

Experience with the design and application of involute helical gears shows that the resources used to employ them have almost been exhausted. Attempts to localize bearing contact had been concentrated on profile modification, but such efforts could not reduce the noise level. Even though the geometry of involute helical gears presents 


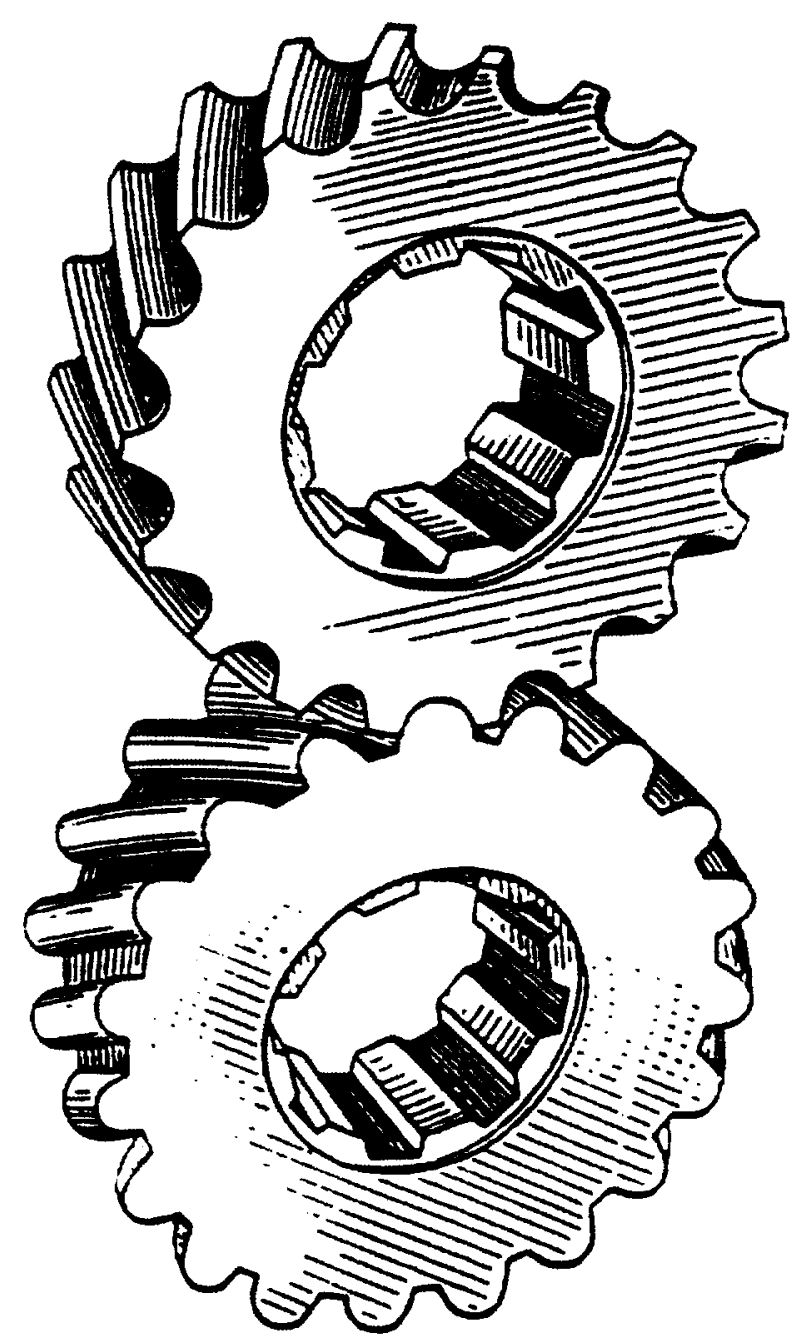

Figure 1.1.-Novikov gears of previous design.

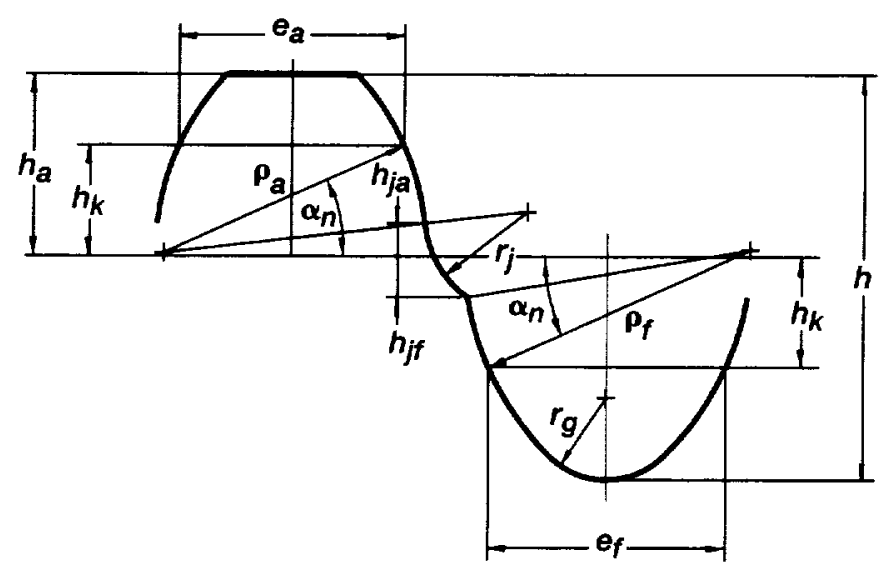

Figure 1.2.-Profiles of rack-cutter for Novikov gears with zones of meshing. 


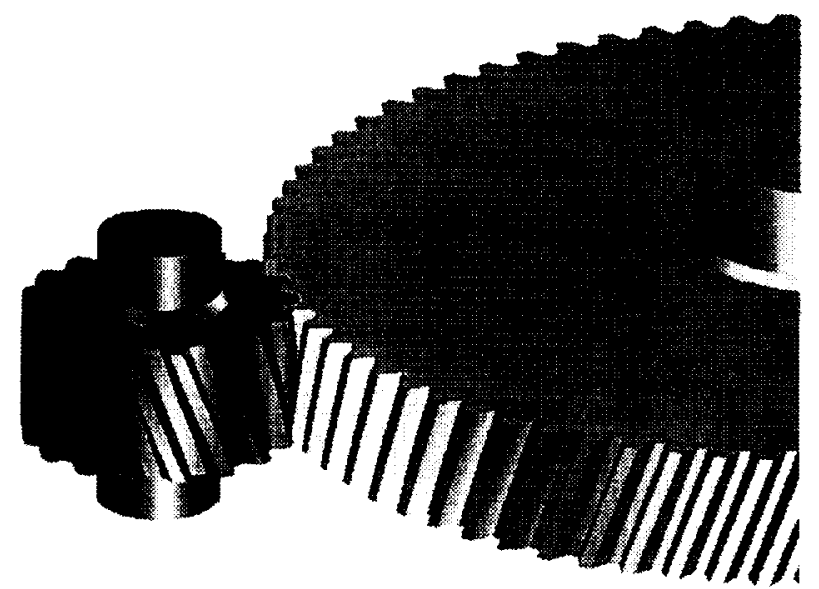

Figure 1.3.-Helical gears with new geometry.

an obstacle in the reduction of contact stresses and noise, they are still an example of high-precision gear drives because of the possibility of grinding by a worm or form grinding.

The new type of helical gears developed by the authors of this report has presented new possibilities for the design and manufacture of low-noise, increased-strength helical gear drives. Although the details of this development are covered in sections 2.0 to 5.0 , a brief summary of the project follows:

1. The bearing contact is localized and the contact stresses are reduced because of the tangency of concaveconvex tooth surfaces of the mating gears. Two versions of the proposed design were investigated (see secs. 2 to 4): one has a convex pinion tooth surface and the other a concave. Figure 1.3 is a three-dimensional view of the designed helical gears with convex pinion teeth.

2. The conjugation of pinion-gear teeth is based on the application of two imaginary rack-cutters with mismatched surfaces $\Sigma_{c}$ and $\Sigma_{t}$ (see details in subsec. 2.1). The normal section of each rack-cutter is a parabola as shown in figure 1.4. A current point of the parabola is determined in an auxiliary coordinate system $S_{i}$ by the equations

$$
x_{i}=u_{i}, \quad y_{i}=a_{i} u_{i}^{2}
$$

where $u_{i}$ is a variable parameter that determines the location of the current point in the normal section and $a_{i}$ is the parabolic coefficient.

Henceforth, we designate by an abbreviated symbol $S_{k}$ an orthogonal coordinate system $S_{k}\left(x_{k}, y_{k}, z_{k}\right)$ where $\left(x_{k}\right.$, $y_{k}, z_{k}$ ) indicate the coordinate axes of $S_{k}$.

3. The normal profiles of mating rack-cutters are in tangency at reference point $Q$, and axis $y_{i}$ (fig. 1.4) is the normal to the profiles at $Q$. The normal profiles are mismatched (fig. 1.5) because different parabolic coefficients $a_{i}$ for the mating rack-cutters have been applied. Points $Q$ of various normal sections of the mating rack-cutters form a line of tangency of their surfaces $\Sigma_{c}$ and $\Sigma_{r}$. It is imagined that rack-cutter surfaces are rigidly connected to each other and generate separately the pinion and gear tooth surfaces. The generated pinion-gear tooth surfaces are in a point contact that spreads under load over an elliptical area (ref. 5).

4. The advantages of applying rack-cutter parabolic profiles in comparison with applying circular-arc profiles in previous designs are as follows:

a. The possibility of increasing the height of the pinion addendum that is limited by tooth pointing and the height of the pinion dedendum that is limited by the possibility of undercutting

b. The possibility of increasing the tooth thickness at the point of tangency of the active profile with the fillet and reducing the bending stresses

These advantages are illustrated by figure 1.6, which shows parabolic profile 1 and circular-arc profile 2 with the same curvature radius $\rho_{1}$ at point $Q$. Point $C_{1}$ is the center of the circle of radius $\rho_{1} ; \alpha$ is the profile angle at $Q$. 


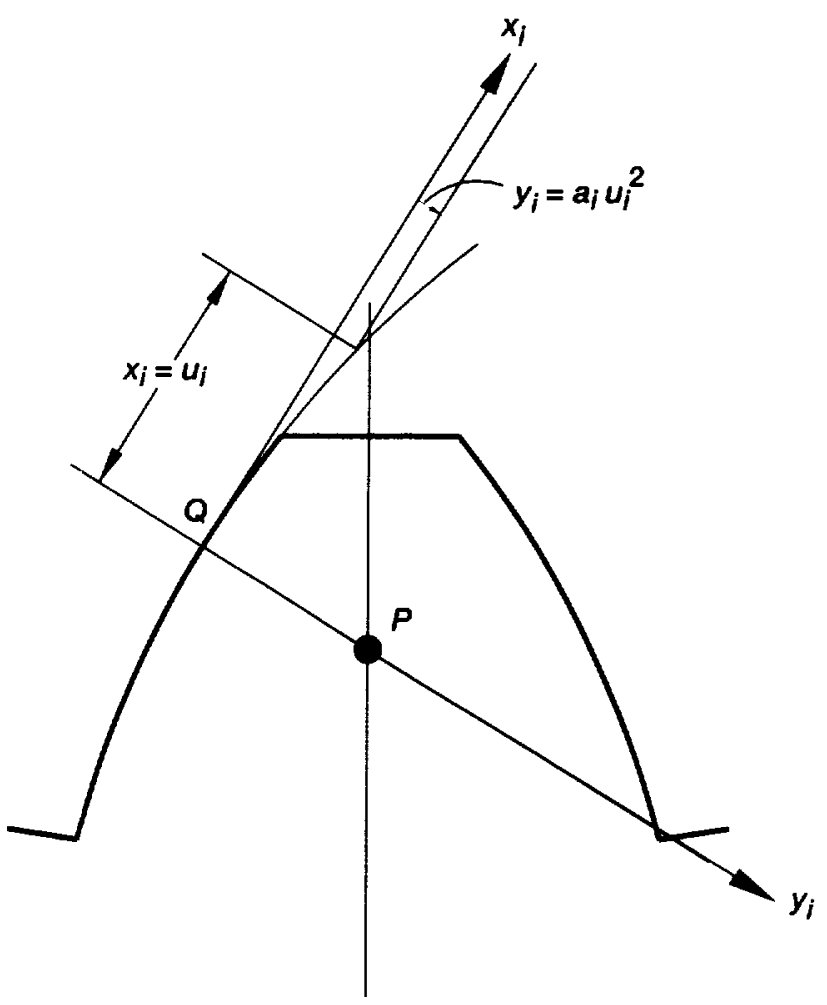

Figure 1.4.-Parabolic profile of rack-cutter in normal section. (a)

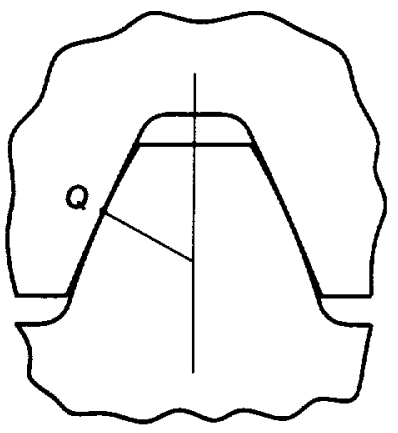

(b)
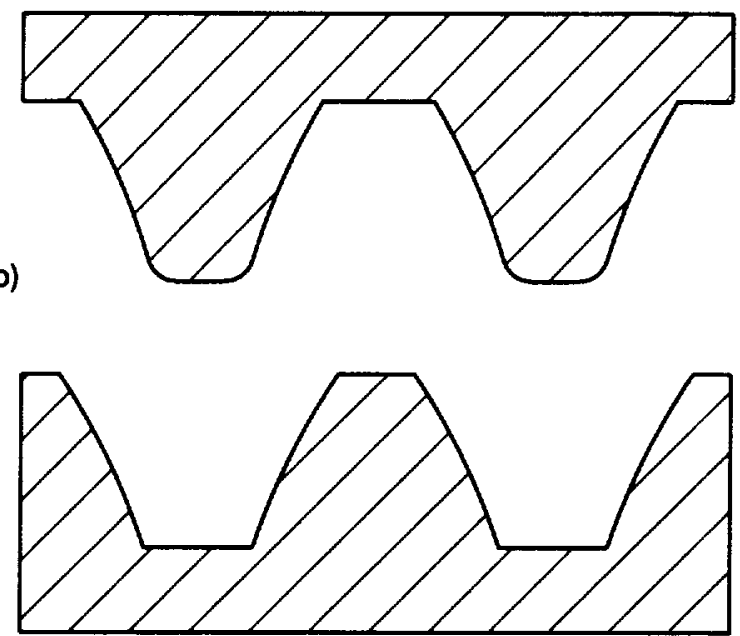

(c)

Figure 1.5.-Rack-cutter applied for generation. (a) Normal profiles of rack-cutters. (b) and (c) Normal sections of pinion and gear rack-cutters.

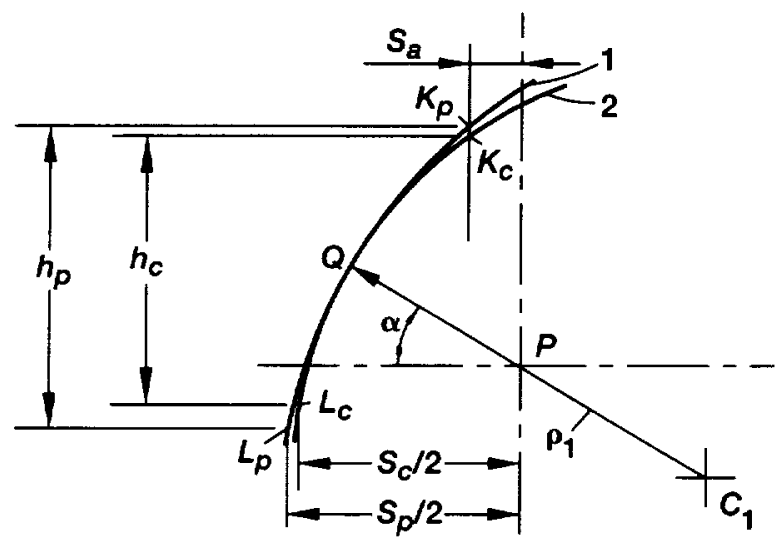

Figure 1.6. - Comparison of parabolic (1) and circular (2) profiles of rack-cutters. 
Point $P$ is the point of intersection of the normal to the tooth profile with the axis of the tooth symmetry and is located on the instantaneous axis of rotation. Points $L_{p}$ and $L_{c}$ indicate limiting points of tooth profiles (for the new and previous design, respectively) obtained from the condition of no undercutting. Similarly, $K_{p}$ and $K_{c}$ indicate limiting points of tooth profiles obtained from the condition of pointing determined for tooth profiles with the same value of $S_{a}$. Parameters $h_{p}$ and $h_{c}$ are the possible tooth heights for the new and previous designs, respectively. Dimensions $S_{p}$ and $S_{c}$ are the possible tooth thicknesses at the point of tangency of the active profile and the fillet for the new and previous designs, respectively.

5. The curvature radii of the rack-cutter profiles for the new gear design are substantially larger than those of the existing design that are based on the application of circular-arc profiles. Thus, the advantages of the new design are that they allow one to

a. Increase the dimensions of the instantaneous contact ellipse and obtain a more favorable orientation, as shown in figure 1.7 where $\mu_{1}$ is the angle between the major axis of the contact ellipse and the tangent to the path of contact on the pinion tooth surface. The center of the contact ellipse coincides with the point of contact $Q$. The dimensions of the contact ellipse have been determined by considering that the elastic approach of tooth surfaces is $\delta=0.00025$ in.

b. Reduce the sensitivity of the gear drive to a change in the gear center distance $\Delta E$, which causes a shift of the bearing contact to the tooth addendum or dedendum (depending on the sign of $\Delta E$ ).

6. The generation of gears for the new and previous designs is based on the application of two mating hobs or grinding worms that are conjugated to the rack-cutters shown in figure 1.5. Two shaped grinding disks can be applied instead of hobs or grinding worms.

In both designs, the bearing contact of tooth surfaces is localized (the tooth surfaces are in point contact at every instant).

7. The main differences in the generation methods for both designs are

a. Only profile crowning is provided in the generation of gears for the previous design. Therefore, the function of transmission errors is almost a linear discontinuous one for a misaligned gear drive (fig. 1.8(a)). Such transmission errors cause vibration for Novikov-Wildhaber gears when the meshing of one pair of teeth is changed for another one.
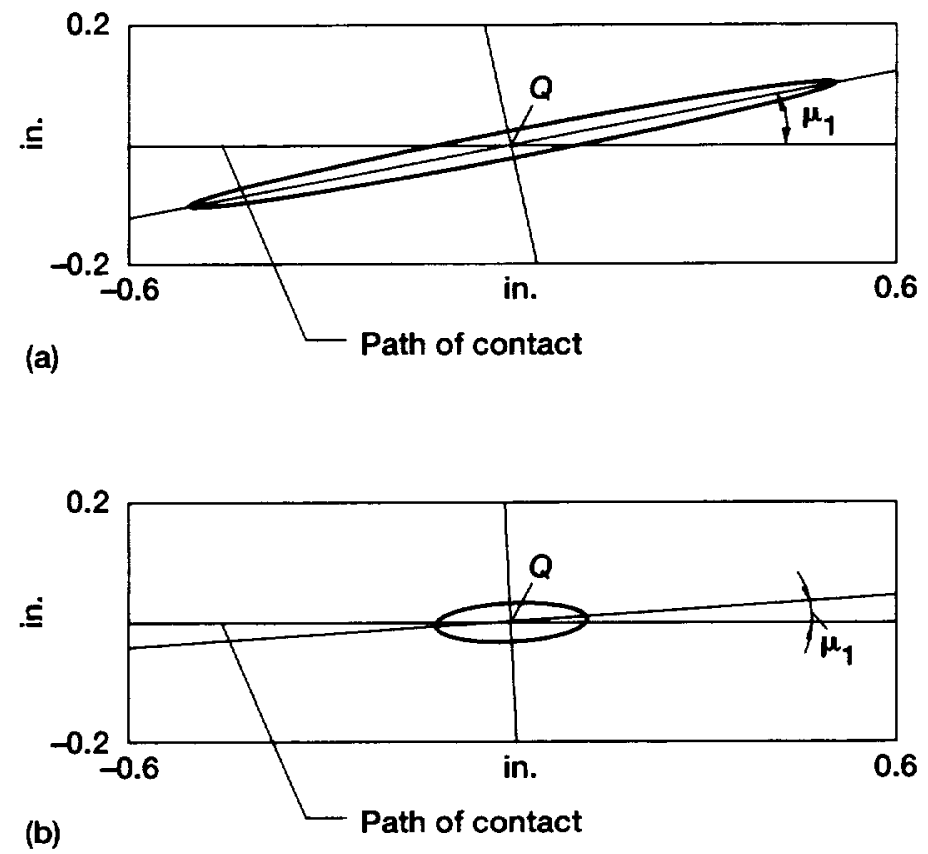

Figure 1.7.-Contact ellipse on pinion tooth surface for (a) proposed design and (b) previous design. 


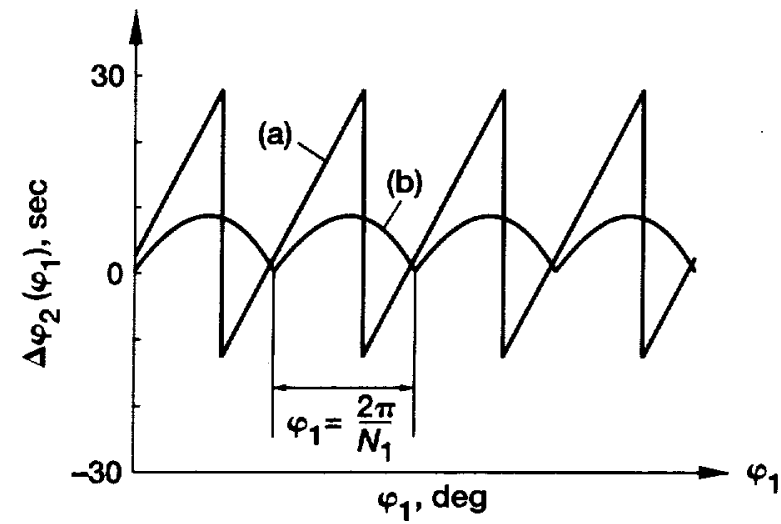

Figure 1.8.-Functions of transmission errors. (a) Linear function of transmission errors caused by error of shaft angle $\Delta \gamma=3$ arc min. (b) Reduction and transformation of errors provided by new geometry of tooth surfaces.
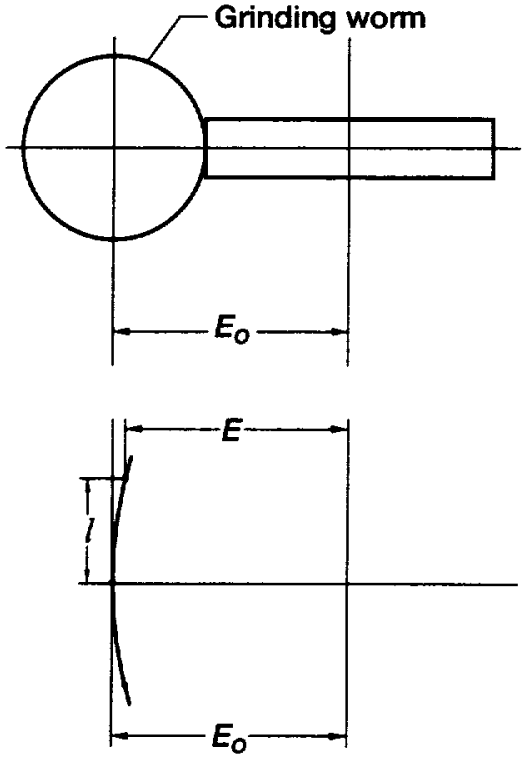

Figure 1.9.-Plunging of grinding worm.

b. The new design provides crowning in two directions: profile and longitudinal. The longitudinal crowning is obtained by the varied plunge of the tool when a grinding worm is applied (fig. 1.9). The shortest distance $E$ between the axes of the grinding worm and the workpiece is represented by

$$
E(l)=E_{0}-a l^{2}
$$

where $l$ is the displacement along the axis of the workpiece; $E_{0}$ is the nominal value of the shortest axis distance; and $a$ is the parabolic coefficient. A parabolic function $E(l)$ must also be provided for generation by hobbing and form grinding.

The varied plunge makes it possible to provide a predesigned parabolic function of transmission errors (fig. 1.8(b)) that can absorb the linear functions of transmission errors caused by gear misalignment and also reduce the magnitude of transmission errors (refs. 5 and 6). The varied plunge of the tool is required only for the generation of one of the mating gears, either the pinion or the gear.

8. The absorption of the linear functions of transmission errors by a parabolic function (provided by tool plunging) is confirmed by tooth contact analysis (TCA) for gears of the new design (see sec. 2.8). The reduction of noise and vibration in the new gears means that they can be used in high-speed transmissions.

9. The misalignment of the new gears may cause a shift in the bearing contact to the edge (fig. 1.10). Drawings in figure 1.10 show the contact path for the following cases: (a) without errors of alignment; (b) a change in the gear center distance $\Delta E$ of $0.1 \mathrm{~mm}$; (c) a change in the shaft angle $\Delta \gamma$ of 3 arc min; (d) a change in the lead angle $\Delta \lambda$ of 3 arc min.

The authors have developed a method to correct the contact path (and the bearing contact) by correcting the lead angle of the pinion or the gear. Because of the existence of a predesigned parabolic function of transmission errors. correcting the lead angle will allow the location of the contact path to be restored without causing transmission errors.

10. The new geometry of helical gears can be extended to conventional involute helical gears as follows: (a) one of the imaginary rack-cutter surfaces is a plane that generates a conventional screw involute surface; (b) the mating rack-cutter is provided with a parabolic profile in the normal section (fig. 1.4) but with a very small curvature; (c) the crowning in the longitudinal direction is accomplished by tool plunging (fig. 1.9), making it possible to obtain a predesigned parabolic function. 

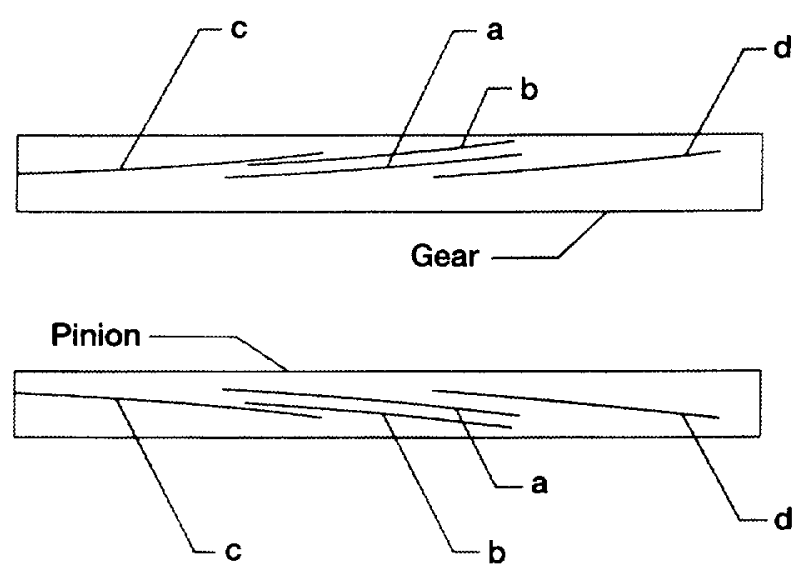

Figure 1.10.-Contact paths on pinion and gear surfaces for one cycle of meshing obtained for helical gears with new geometry. (a) Without errors of alignment. (b) Change of center distance $\Delta E=0.1 \mathrm{~mm}$. (c) Change of shaft angle $\Delta \gamma=3$ arc min. (d) Change of lead angle $\Delta \lambda=3$ arc min.

The advantage of the proposed modification of conventional involute helical gears is the possibility of reducing the magnitude of transmission errors and transforming their shape (see plot (b) in fig. 1.8).

This report covers

1. The computerized design and imaginary rack-cutter generation of two versions of profile-crowned helical gears. The two versions are designed as pinion convex-gear concave ( $\mathrm{sec} .2 .0$ ) and pinion concave-gear convex (sec. 3.0). The designed gearing is a new type of Novikov-Wildhaber helical gear.

2. The avoidance of undercutting and tooth pointing (secs. 2.0 and 3.0).

3. The grinding (or cutting) of the designed helical gears crowned in the profile and longitudinal directions by the application of a disk or worm (sec. 4.0).

4. The computerized simulation of the meshing and contact of the designed helical gears crowned in (a) the profile direction only, and (b) the profile and longitudinal directions (secs. 2.0 to 4.0).

\subsection{HELICAL PINION CONVEX AND GEAR CONCAVE PROFILE-CROWNED TOOTH SURFACES}

\subsection{Basic Considerations}

This section covers the geometry, generation, design, and simulation of the meshing and contact of profilecrowned tooth surfaces. The pinion and gear tooth surfaces are convex and concave. Section 3.0 discusses another version of tooth surfaces, pinion concave and gear convex.

The generation of pinion-gear tooth surfaces is based on the following ideas:

1. Two imaginary rigidly connected rack-cutters for the separate generation of the pinion and the gear are applied (fig. 1.5).

2. The axodes of the pinion and the gear in mesh with the rack-cutters are two pitch cylinders of radii $r_{p l}$ and $r_{p 2}$ (fig. 2.1.1(a)). The axode of the rigidly connected rack-cutters is plane $\Pi$ that is tangent to the pitch cylinders. The pinion and gear perform rotational motions about their axes whereas the rack-cutters perform translational motions, all of which are related. The pitch cylinders and plane $\Pi$ are in tangency at line $P-P$, which is the instantaneous axis of rotation in relative motion.

3. The rack-cutters are provided with skew teeth (fig. 2.1.1(b) and from the drawings, it is obvious that a lefthand rack-cutter generates a left-hand pinion and a right-hand gear. The helix angles on the pinion-gear pitch cylinders are equal to angle $\beta$ of the skew teeth of the rack-cutters. 


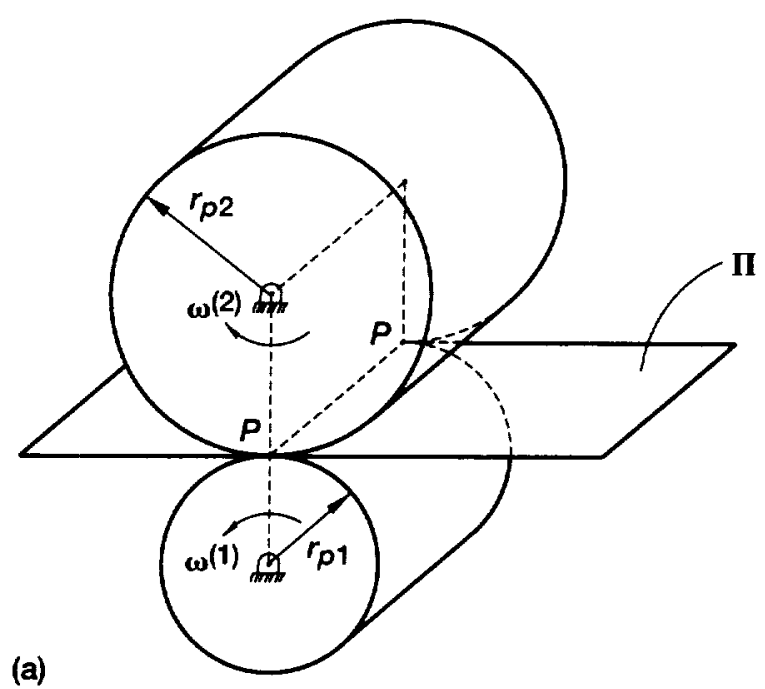

(a)

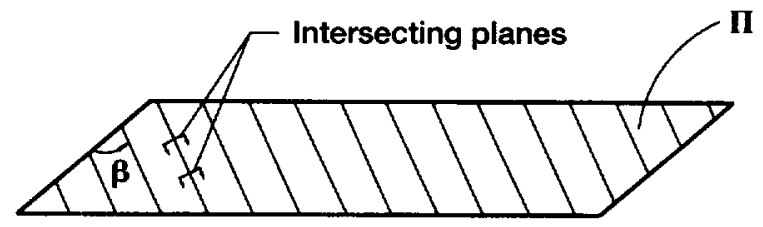

(b)

Figure 2.1.1.-Axodes of pinion, gear, and rack-cutter.

(a) Axodes. (b) Direction of rack-cutter teeth.

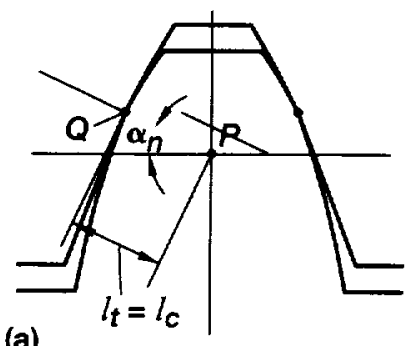

(a)

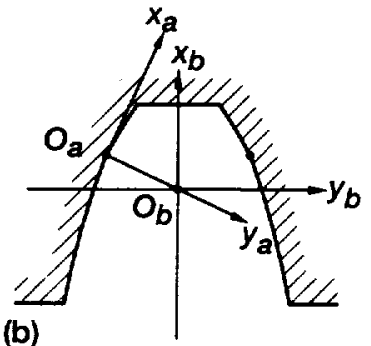

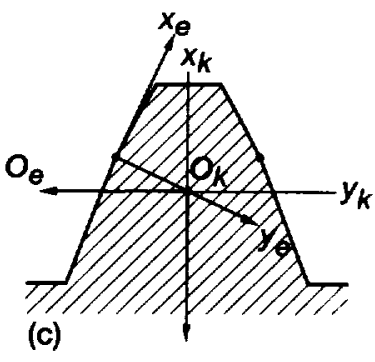

(c)
Figure 2.1.2.-Normal sections of rack-cutters

(a) Profiles of pinion and gear rack-cutters.

(b) Pinion rack-cutter. (c) Gear rack-cutter.

4. The normal profiles of rack-cutters are obtained by the intersection of rack-cutter surfaces by a vertical plane that is perpendicular to the direction of rack-cutter teeth. The normal profiles are two mismatched parabolas in tangency at point $Q$ (fig. 2.1.2(a)), and their common normal passes through point $P$ that belongs to the instantaneous axis of rotation $P$ - $P$ (fig. 2.1.1(a)).

5. Using various intersecting normal planes (fig. 2.1.1(b)) results in the same rack-cutter normal profiles shown in figure 2.1.2(a). Henceforth, we will consider two rack-cutter surfaces $\Sigma_{c}$ and $\Sigma$, that generate the pinion and gear tooth surfaces $\Sigma_{1}$ and $\Sigma_{2}$, respectively. Surfaces $\Sigma_{c}$ and $\Sigma_{t}$ are in tangency along a straight line that passes through points $Q$ of tangency of various normal profiles. The orientation of the line of tangency of $\Sigma_{c}$ and $\Sigma_{t}$ is determined by angle $\beta$.

Because the rack-cutter surfaces are mismatched, the pinion-gear tooth surfaces are in point contact (but not in line contact) and therefore the bearing contact is localized.

\subsection{Derivation of Pinion Tooth Surface $\Sigma_{1}$}

Pinion rack-cutter surface $\Sigma_{c}$.-The derivation of rack-cutter surface $\Sigma_{c}$ is based on the following procedure:

Step 1: The normal profile of $\Sigma_{c}$ is a parabola and is represented in coordinate system $S_{a}$ (fig. 2.1.2(b)) by equations that are similar to equations $(1.1)$ :

$$
\mathbf{r}_{a}\left(u_{c}\right)=\left[\begin{array}{lllll}
u_{c} & a_{c} u_{c}^{2} & 0 & 1
\end{array}\right]^{T}
$$

where $a_{c}$ is the parabolic coefficient. 
Step 2: The normal profile is represented in $S_{b}$ by matrix equation

$$
\mathbf{r}_{b}\left(u_{c}\right)=\mathbf{M}_{b a} \mathbf{r}_{a}\left(u_{c}\right)
$$

The distance $\left|\overline{O_{a} O_{b}}\right|$ is designated as $l_{c}$, and $\mathbf{M}_{b a}$ indicates the $4 \times 4$ matrix used for the coordinate transformation from coordinate system $S_{a}$ to $S_{b}$ (ref. 7). Homogeneous coordinates of a point are considered in reference 7 .

Step 3: Consider that rack-cutter surface $\Sigma_{c}$ is formed in $S_{c}$ while coordinate system $S_{b}$ with the normal profile performs a translational motion in the direction $a$ - $a$ of the skew teeth of the rack-cutter (fig. 2.2.1).

Surface $\Sigma_{c}$ is determined in coordinate system $S_{c}$ in two-parameter form by the following matrix equation:

$$
\mathbf{r}_{c}\left(u_{c}, \theta_{c}\right)=\mathbf{M}_{c b}\left(\theta_{c}\right) \mathbf{M}_{b a} \mathbf{r}_{a}\left(u_{c}\right)=\mathbf{M}_{c a}\left(\theta_{c}\right) \mathbf{r}_{a}\left(u_{c}\right)
$$

where $\theta_{c}=\left|\overline{O_{c} O_{b}}\right|$ (fig. 2.2.1) and $\left(u_{c}, \theta_{c}\right)$ are surface parameters.

Step 4: The normal $\mathbf{N}_{c}$ to rack-cutter surface $\Sigma_{c}$ is determined by matrix equation (ref. 7)

$$
\mathbf{N}_{c}\left(u_{c}, \theta_{c}\right)=\mathbf{L}_{c b}\left(\theta_{c}\right) \mathbf{L}_{b a} \mathbf{N}_{a}\left(u_{c}\right)
$$

Here

$$
\mathbf{N}_{a}\left(u_{c}\right)=\mathbf{k}_{a} \times \frac{\partial \mathbf{r}_{a}}{\partial u_{c}}
$$

where $\mathbf{L}_{c b}$ indicates the $3 \times 3$ matrix that is the submatrix of $\mathbf{M}_{c b}$ and is used for the transformation of vector components; $\mathbf{k}_{a}$ is the unit vector of axis $z_{a}$.

The transverse section of rack-cutter $\Sigma_{c}$ is shown in figs. 2.2.2(a) and (b).

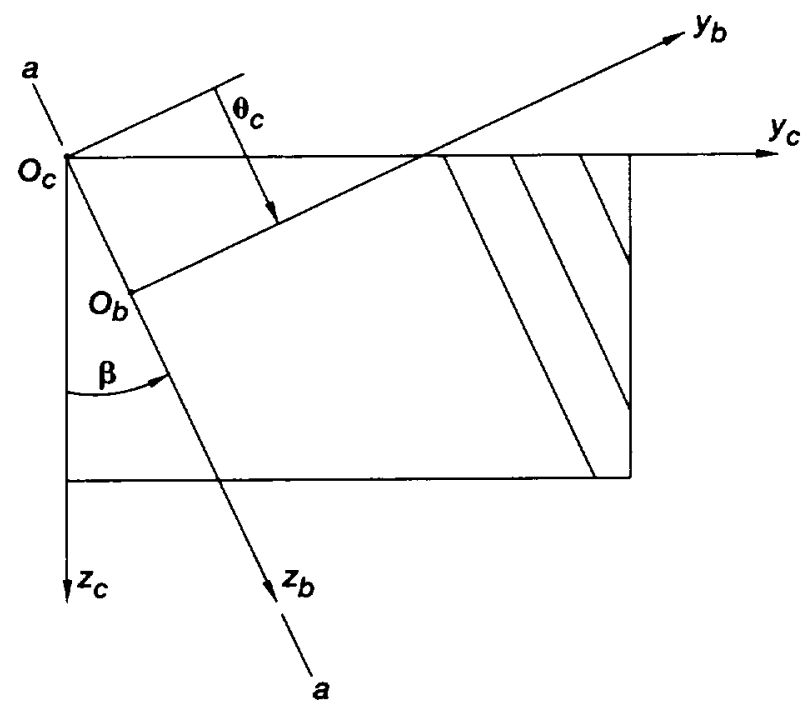

Figure 2.2.1.-For derivation of pinion rack-cutter surface $\Sigma_{c}$.

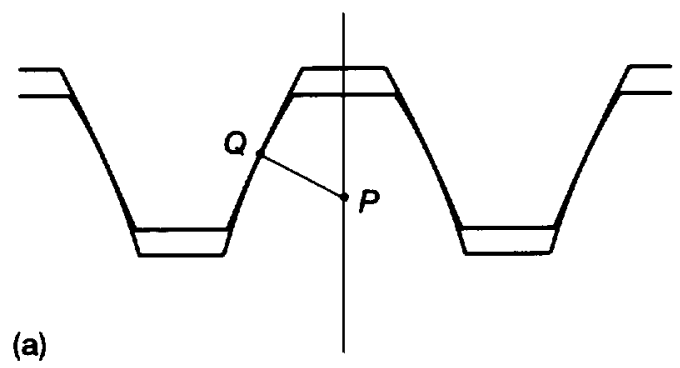

(a)
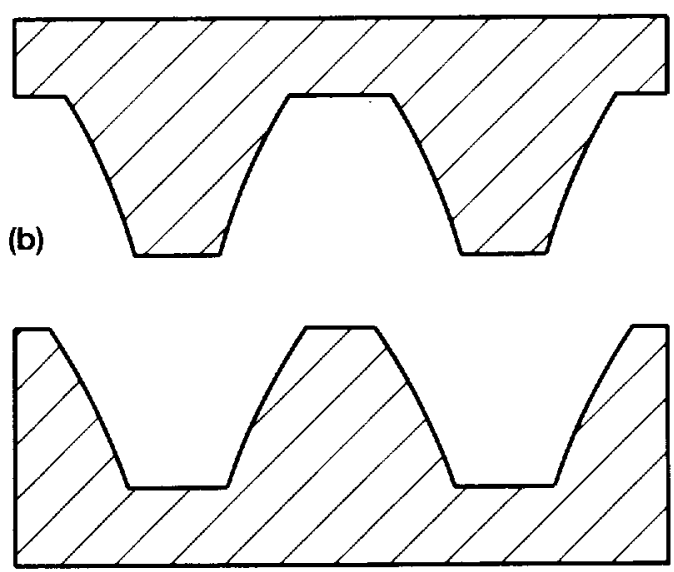

(c)

Figure 2.2.2.-Rack-cutter transverse profiles. (a) Mating profiles. (b) Pinion rack-cutter profile. (c) Gear rack-cutter profile. 


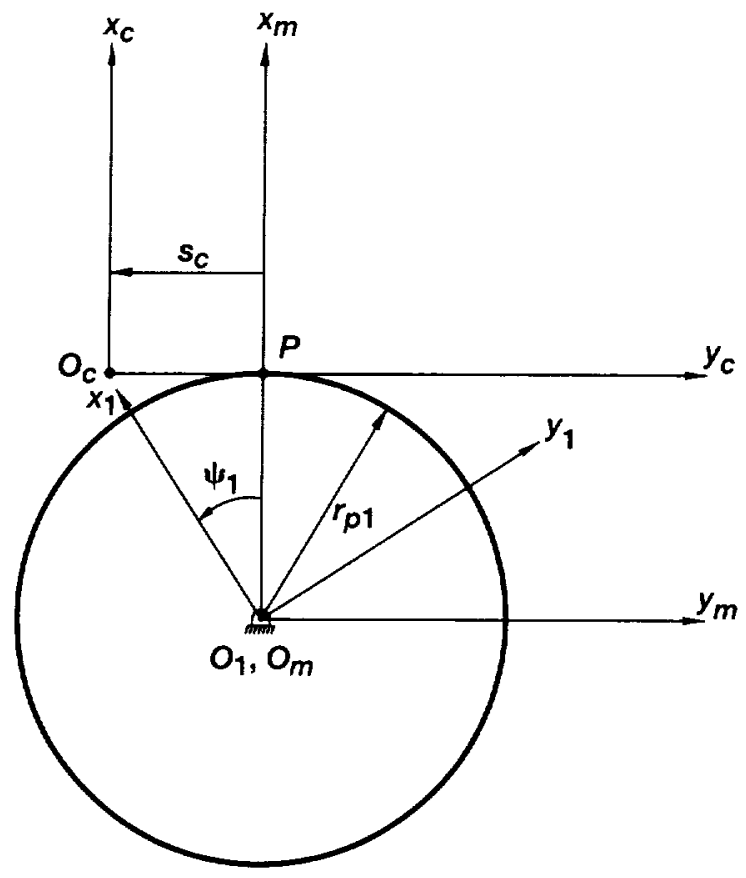

Figure 2.2.3.-Generation of pinion by rack-cutter $\Sigma_{c}$.

Determination of pinion tooth surface $\Sigma_{1}$. - The determination of $\Sigma_{1}$ is based on the following considerations:

1. Movable coordinate systems $S_{c}$ and $S_{1}$ (fig. 2.2.3) are rigidly connected to the pinion rack-cutter and the pinion, respectively. The fixed coordinate system $S_{m}$ is rigidly connected to the cutting machine.

2. The rack-cutter and the pinion perform related motions, as shown in figure 2.2.3 where $s_{c}=r_{p 1} \psi_{1}$ is the displacement of the rack-cutter in its translational motion, and $\psi_{1}$ is the angle of rotation of the pinion.

3. A family of rack-cutter surfaces is generated in coordinate system $S_{1}$ and is determined by the matrix equation

$$
\mathbf{r}_{1}\left(u_{c}, \theta_{c}, \psi_{1}\right)=\mathbf{M}_{1 c}\left(\psi_{1}\right) \mathbf{r}_{c}\left(u_{c}, \theta_{c}\right)
$$

4. The pinion tooth surface $\Sigma_{1}$ is generated as the envelope to the family of surfaces $\mathbf{r}_{1}\left(u_{c}, \theta_{c}, \Psi_{1}\right)$. Surface $\Sigma_{1}$ is determined by simultaneous consideration of vector function $\mathbf{r}_{1}\left(u_{c}, \theta_{c}, \psi_{1}\right)$ and the so-called equation of meshing:

$$
f_{l c}\left(u_{c}, \theta_{c}, \psi_{1}\right)=0
$$

5. To derive the equation of meshing (2.2.7), apply the following theorem (refs. 5 and 7): The common normal to surfaces $\Sigma_{c}$ and $\Sigma_{l}$ at their line of tangency must pass through the instantaneous axis of rotation $P$ (fig. 2.2.3). The result is

$$
\frac{X_{c}-x_{c}}{N_{x c}}=\frac{Y_{c}-y_{c}}{N_{y c}}=\frac{Z_{c}-z_{c}}{N_{z c}}
$$

where $\left(x_{c}, y_{c}, z_{c}\right)$ are the coordinates of a current point of $\Sigma_{c} ;\left(N_{x c}, N_{y c}, N_{z c}\right)$ are the components of the normal to $\Sigma_{c}$; $X_{c}=0, Y_{c}=r_{p 1} \psi_{1}$, and $\Sigma_{c}$ are the coordinates of the intersection point of normal $\mathbf{N}_{c}$ with the instantaneous axis of rotation $P$ (fig. 2.2.3).

To derive the equation of meshing (2.2.7), it is sufficient to consider equation

$$
-\frac{x_{c}\left(u_{c}, \theta_{c}\right)}{N_{x c}\left(u_{c}, \theta_{c}\right)}=\frac{r_{p 1} \Psi_{1}-y_{c}\left(u_{c}, \theta_{c}\right)}{N_{y c}\left(u_{c}, \theta_{c}\right)}
$$


that yields

$$
f_{1 c}\left(u_{c}, \theta_{c}, \psi_{1}\right)=\left(r_{p 1} \psi_{1}-y_{c}\right) N_{x c}+x_{c} N_{y c}=0
$$

Equations (2.2.6) and (2.2.10) represent the pinion tooth surface by three related parameters. Taking into account that the equations above are linear with respect to $\theta_{c}$, we may eliminate $\theta_{c}$ and represent the pinion tooth surface by vector function $\mathbf{r}_{1}\left(u_{c}, \Psi_{1}\right)$.

\subsection{Derivation of Gear Tooth Surface $\Sigma_{2}$}

Gear rack-cutter surface $\Sigma_{t}$-The derivation of $\Sigma_{t}$ is based on the procedure similar to that applied for the derivation of $\Sigma_{c}$ (see eqs. (2.2.3)). The normal profile of $\Sigma_{t}$ is a parabola represented in $S_{e}$ by equation (fig. 2.1.2(c))

$$
\mathbf{r}_{e}\left(u_{t}\right)=\left[\begin{array}{llll}
u_{t} & a_{t} u_{t}^{2} & 0 & 1
\end{array}\right]^{T}
$$

which is similar to equation (2.2.1). Use coordinate systems $S_{k}$ (fig. 2.1.2(c)) and $S_{t}$ that are similar to $S_{b}$ and $S_{c}$ (fig. 2.2.1) to represent surface $\Sigma_{t}$ by matrix equation

$$
\mathbf{r}_{t}\left(u_{t}, \theta_{t}\right)=\mathbf{M}_{t k}\left(\theta_{t}\right) \mathbf{M}_{k e} \mathbf{r}_{\mathbf{e}}\left(u_{t}\right)=\mathbf{M}_{t e} \mathbf{r}_{\mathbf{e}}\left(u_{t}\right)
$$

Equation (2.3.2) can be used to represent surface $\Sigma_{t}$ by vector function $\mathbf{r}_{t}\left(u_{t}, \theta_{t}\right)$, which is similar to $\mathbf{r}_{c}\left(u_{c}, \theta_{c}\right)$. The difference in the representation of $\Sigma_{t}$ is the change in the subscript $c$ to $t$.

The normal to surface $\Sigma_{t}$ is determined by equations similar to (2.2.4) and (2.2.5).

Gear tooth surface $\Sigma_{2}$. - The generation of $\Sigma_{2}$ by rack-cutter surface $\Sigma_{t}$ is represented schematically in figure 2.3.1. The rack-cutter and the gear perform related translational and rotational motions designated as $s_{t}=r_{p 2} \psi_{2}$ and $\psi_{2}$.

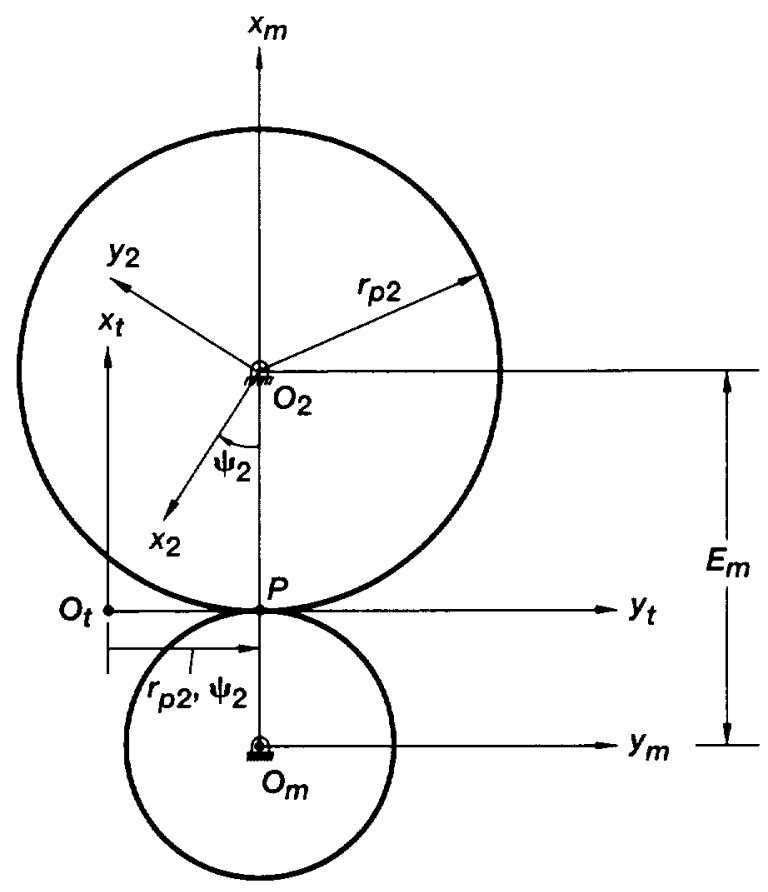

Figure 2.3.1.-Generation of gear by rack-cutter $\Sigma_{t}$. 
The gear tooth surface is represented by equations

$$
\begin{aligned}
& \mathbf{r}_{2}=\mathbf{r}_{2}\left(u_{t}, \theta_{t}, \psi_{2}\right) \\
& f_{2 t}\left(u_{t}, \theta_{t}, \psi_{2}\right)=0
\end{aligned}
$$

Equation (2.3.3) represents in $S_{2}$ the family of rack-cutter surfaces $\Sigma_{t}$ determined as

$$
\mathbf{r}_{2}\left(u_{t}, \theta_{t}, \psi_{2}\right)=\mathbf{M}_{2 t}\left(\psi_{2}\right) \mathbf{r}_{t}\left(u_{t}, \theta_{t}\right)
$$

The derivation of the equation of meshing (2.3.4) may be accomplished similarly to that of equation (2.2.10)

$$
f_{2 t}\left(u_{t}, \theta_{t}, \Psi_{2}\right)=\left(r_{p 2} \Psi_{2}-y_{t}\right) N_{x t}+x_{t} N_{y t}=0
$$

Drawings of figs. 2.1.1 and 2.3.1 show that a left-hand rack-cutter generates a right-hand gear. Equations (2.3.5) and (2.3.6) represent the gear tooth surface by three related parameters. The linear parameter $\theta_{t}$ can be eliminated and the gear tooth surface represented in two-parameter form by vector function $\mathbf{r}_{2}\left(u_{t}, \psi_{2}\right)$.

\subsection{Avoidance of Undercutting and Pointing}

The generation of a helical tooth surface by a rack-cutter may be accompanied by undercutting of the surface being generated. The discovery of undercutting is based on the following theorem proposed in references 5 and 7 : Singular points of the generated surface $\Sigma$ occur when the velocity of a contact point in its motion over $\Sigma$ becomes equal to zero. Note that at a singular surface point, the surface normal is equal to zero. Thus, the appearance of a singular point heralds the oncoming of surface undercutting.

References 5 and 8 propose an approach for determining a line $L$ of regular points on the tool surface that generates singular points on the generated surface. The dimensions of the tool surface must be limited by line $L$ to avoid undercutting. In the case of generation by a rack-cutter, the limiting line $L$ of the rack-cutter surface determines the limiting height of the rack-cutter addendum.

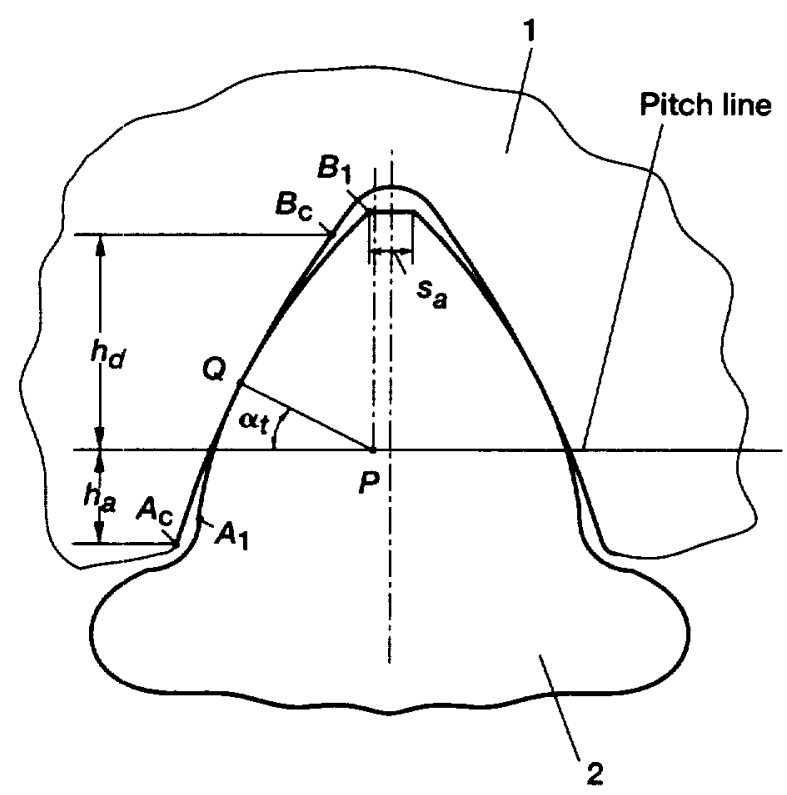

Figure 2.4.1.-For determination of conditions of undercutting and pointing by rack-cutter.

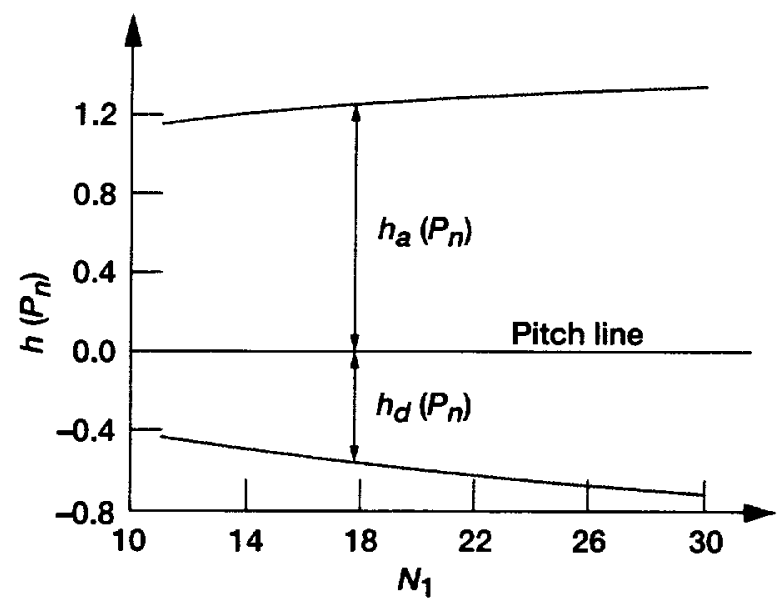

Figure 2.4.2.-Permissible dimensions of rack-cutter addendum $h_{a}$ and dedendum $h_{d}$. 
The pointing of generated teeth means that the topland width of the generated teeth becomes equal to zero. In the present project, pointing was avoided because a minimal value of the topland width was provided.

Figure 2.4.1 shows the transverse profiles of the rack-cutter space and the profiles of the generated pinion tooth. Point $A_{c}$ is the limiting one of the rack-cutter left profile that generates the singular point $A_{1}$ of the left pinion transverse profile. Point $B_{c}$ of the rack-cutter profile generates point $B_{1}$ of the pinion profile. The width of the pinion topland is designated by $S_{a}$. The left transverse profiles of the rack-cutter and the pinion are shown at a position where they are in tangency at point $Q$. Point $P$ belongs to the instantaneous axis of rotation. The heights $h_{a}$ and $h_{d}$ of the working parts of the rack-cutter addendum and dedendum are measured from the pitch line of the rack-cutter.

The results of the present investigation are illustrated by the curves of figure 2.4.2, which represent functions $h_{a}\left(N_{1}\right)$ and $h_{d}\left(N_{1}\right)$ determined for the data $\beta=20^{\circ}, \alpha_{n}=25^{\circ}, 10 \leq N_{1} \leq 30$, and $P_{n}=1 / \mathrm{in}$. These curves were obtained by considering the meshing of the transverse profile of the rack-cutter with the transverse profile of the pinion (or the gear). Note that the normal profile of the rack-cutter is a parabola and the rack-cutter surface $\Sigma_{c}$ is a parabolic cylinder. The transverse profile of the rack-cutter has also been proven to be a parabola. The parabolic coefficients $a_{c}^{*}$ and $a_{c}$ of the transverse and normal profiles are related as

$$
a_{c}^{*}=a_{c} \frac{\cos ^{3} \alpha_{t}}{\cos ^{3} \alpha_{n} \cos \beta}
$$

The surface of action between the rack-cutter surface and the generated pinion (or gear tooth surface) is a cylindrical surface. The transverse section of the surface of action is shown in figure 2.4.3.

In summarizing the results of this investigation, we may state that undercutting may occur for a pinion with a small number of teeth (see fig. 2.4.2). A sufficient working height $h=h_{a}+h_{d}$ of the teeth is in the range $\left(1.5 / P_{n}\right) \leq h \leq\left(1.9 / P_{n}\right)$.

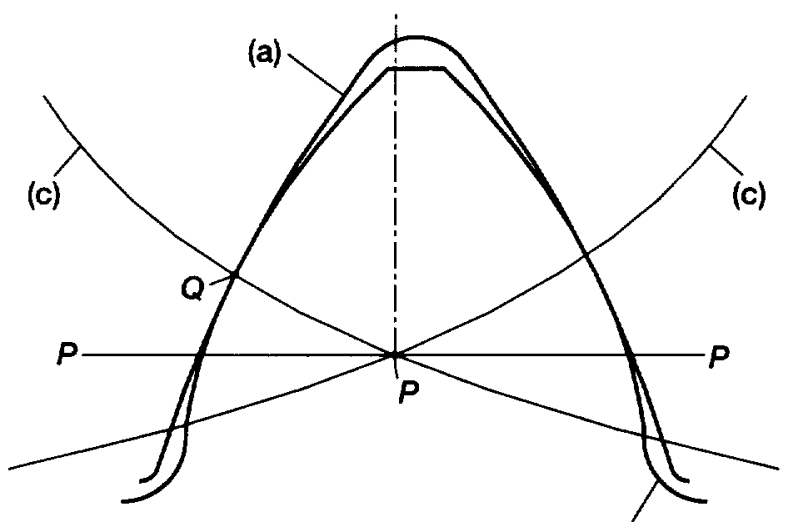

(b)

Figure 2.4.3.-Transverse profiles and lines of action. (a) Rack-cutter profile. (b) Pinion profile. (c) Lines of action.

\subsection{Principal Curvatures and Directions of Pinion-Gear Tooth Surfaces}

Knowledge of principal curvatures and directions of the contacting surfaces $\Sigma_{1}$ and $\Sigma_{2}$ is required to determine the dimensions of the instantaneous contact ellipse. Determining the principal curvatures of surfaces $\Sigma_{1}$ and $\Sigma_{2}$ is based on the approach developed in references 5 and 7 . The advantage of this approach is that the sought-for curvatures are expressed in terms of the principal curvatures and directions of the tool surface and the parameters of motion. In the case of the helical gears under discussion, the principal curvatures and directions of pinion-gear tooth surfaces can be expressed in terms of the principal curvatures and directions of rack-cutters and the parameters of motion. 


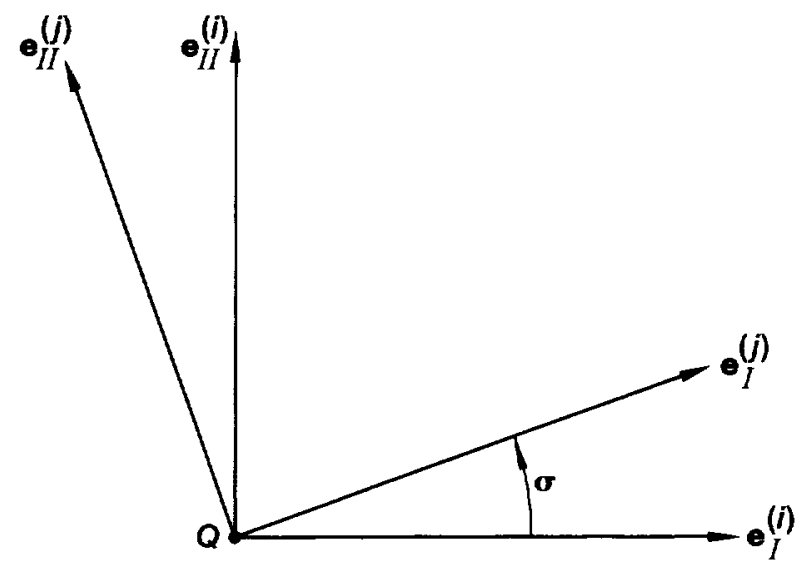

Figure 2.5.1.-Principal directions on generating and generated surfaces; $i=c, t ; j=1,2$.

Let us start the derivations by determining the curvatures of the rack-cutter. The surface of rack-cutter $\Sigma_{c}$ (or $\Sigma_{t}$ ) is a cylindrical surface whose generatrix has the direction of skew rack-cutter teeth. Such a surface is a ruled "developed" surface, which means that it is formed by straight lines (the generatrices of the cylindrical surface), and the normals along the generatrix do not change their direction. The generatrix is the surface principal direction and the principal curvature in this direction is equal to zero. This means that

$$
\kappa_{I}^{(i)}=0 \quad(i=c, t)
$$

The other principal direction is the tangent to the parabola that represents the normal profile and the principal curvature is the curvature of the parabola determined at point $Q$ as

$$
\kappa_{l I}^{(i)}=2 a_{i} \quad(i=c, t)
$$

The principal curvatures and directions of the pinion (or gear) tooth surface generated by the rack-cutter are determined by using the approach developed in references 7 and 8 , which enables us to determine the following: $\sigma$, the angle formed by principal directions $\mathbf{e}_{I}^{(j)}$ and $\mathbf{e}_{I}^{(i)}$ (fig. 2.5.1) and principal curvatures on directions $\mathbf{e}_{I}^{(j)}$ and $\mathbf{e}_{I I}^{(j)}$ on surface $\Sigma_{j}$. Here, $j=1$ when $i=c$ (when pinion tooth surface $\Sigma_{1}$ generated by rack-cutter $\Sigma_{c}$ is considered). Similarly, $j=2$ when $i=t$ (when gear tooth surface $\Sigma_{2}$ generated by rack-cutter $\Sigma_{t}$ is considered).

We have limited the determination of principal curvatures and directions on surfaces $\Sigma_{1}$ and $\Sigma_{2}$ only at point $Q$ of the tangency of the generating and generated surfaces (fig. 2.1.2). In an ideal profile-crowned gear drive, the path of contact on $\Sigma_{1}$ (and $\Sigma_{2}$ ) is a helix generated by point $Q$, and the principal curvatures and directions at any point of the helix are the same.

The approach discussed above is illustrated by an example with the following data: tooth numbers $N_{1}=17$, $N_{2}=77$; the normal profile angle $\alpha_{n}=25^{\circ}$; the normal diametral pitch $P_{n}=5$; the parabolic coefficients $a_{c}=0.425$ and $a_{t}=0.394$; the helix angle $\beta=19.548^{\circ}$.

The results of computing the principal curvatures of $\Sigma_{1}$ and $\Sigma_{2}$ at point $Q$ (fig. 2.1.2) are as follows: $\kappa_{I}^{(1)}=0.0125, \kappa_{I I}^{(1)}=1.5605, \kappa_{I}^{(2)}=0.0083 ;$ and $\kappa_{I I}^{(2)}=0.5940$.

Therefore, for the pinion tooth surface with a convex normal profile, both principal curvatures are positive. The Gaussian curvature $K^{(1)}=\kappa_{I}^{(1)} \kappa_{l l}^{(1)}>0$ and all points of this surface are elliptic ones.

For the gear tooth surface with a normal profile, the principal curvatures have opposite signs. The Gaussian curvature $K^{(2)}=\kappa_{I}^{(2)} \kappa_{I I}^{(2)}>0$ and all points of this surface are hyperbolic. 


\subsection{Determination of Contact Ellipse}

The determination of the instantaneous contact ellipse is based on the following considerations (refs. 5 and 7 ):

1. Consider as known: the principal curvatures and directions on surfaces $\Sigma_{1}$ and $\Sigma_{2}$ and angle $\sigma$ formed by unit vectors $\mathbf{e}_{l}^{(1)}$ and $\mathbf{e}_{I}^{(2)}$ (fig. 2.6.1). The details for determining the principal curvatures of contacting surfaces are given in reference 7 .

2. The elastic deformation $\delta$ of contacting surfaces is given.

Now, determine the orientation of the contact ellipse by using angle $\alpha^{(1)}$ and the dimensions $a$ and $b$ of the contact ellipse and by using the following equations:

$$
\begin{aligned}
& \cos 2 \alpha^{(1)}=\frac{g_{1}-g_{2} \cos 2 \sigma}{\left(g_{1}^{2}-2 g_{1} g_{2} \cos 2 \sigma+g_{2}^{2}\right)^{1 / 2}} \\
& \sin 2 \alpha^{(1)}=\frac{g_{2} \sin 2 \sigma}{\left(g_{1}^{2}-2 g_{1} g_{2} \cos 2 \sigma+g_{2}^{2}\right)^{1 / 2}}
\end{aligned}
$$

Axes of the contact ellipse are determined with equations

$$
2 a=2\left|\frac{\delta}{A}\right|^{1 / 2}, \quad 2 b=2\left|\frac{\delta}{B}\right|^{1 / 2}
$$

where

$$
\begin{aligned}
& A=\frac{1}{4}\left[\kappa_{\Sigma}^{(1)}-\kappa_{\Sigma}^{(2)}-\left(g_{1}^{2}-2 g_{1} g_{2} \cos 2 \sigma+g_{2}^{2}\right)^{1 / 2}\right] \\
& B=\frac{1}{4}\left[\kappa_{\Sigma}^{(1)}-\kappa_{\Sigma}^{(2)}+\left(g_{1}^{2}-2 g_{1} g_{2} \cos 2 \sigma+g_{2}^{2}\right)^{1 / 2}\right]
\end{aligned}
$$

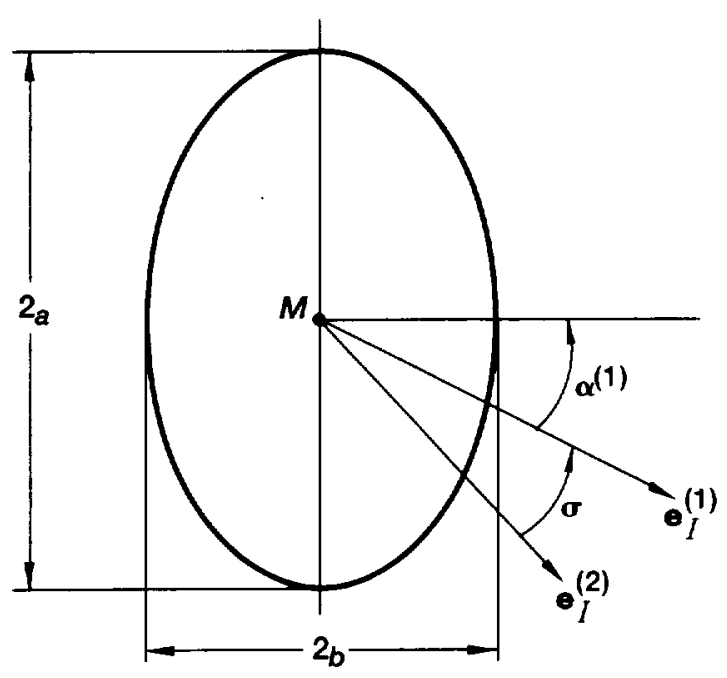

Figure 2.6.1.-Contact ellipse. 

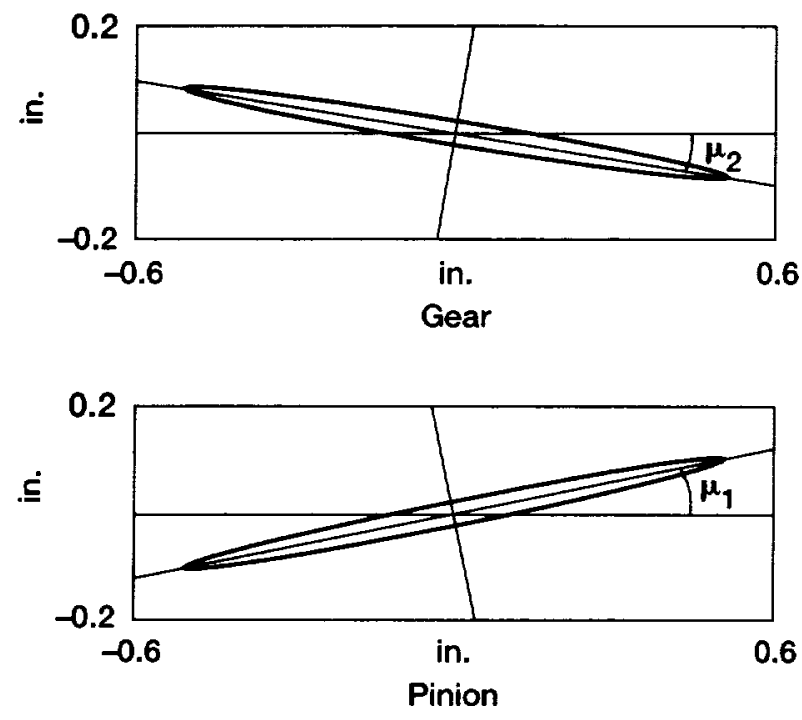

Figure 2.6.2.-Contact ellipses on pinion and gear tooth surfaces.

$$
\kappa_{\Sigma}^{(i)}=\kappa_{I}^{(i)}+\kappa_{I I}^{(i)}, \quad g_{i}=\kappa_{I}^{(i)}-\kappa_{I I}^{(i)}, \quad(i=1,2)
$$

Figure 2.6.2 shows the contact ellipses determined on pinion and gear tooth surfaces $\Sigma_{1}$ and $\Sigma_{2}$. To avoid misunderstanding, we have to emphasize that the tangents to contact paths on the mating rack-cutter surfaces $\Sigma_{c}$ and $\Sigma_{t}$ coincide with each other (they are directed along the line of tangency of $\Sigma_{c}$ and $\Sigma_{t}$ ). However, the tangents to the contact paths on pinion-gear tooth surfaces $\Sigma_{1}$ and $\Sigma_{2}$ at point $Q$ of surface tangency slightly differ from each other. Therefore, the orientation of contact ellipses is determined by different angles $\mu_{1}$ and $\mu_{2}$, as shown in figure 2.6.2. The dimensions and orientation of the contact ellipse have been determined for these design parameters: tooth numbers $N_{1}=17$ and $N_{2}=77$; normal profile angle $\alpha_{n}=25^{\circ}$; normal diametral pitch $P_{n}=5$; parabolic coefficients $a_{c}=0.425$ and $a_{t}=0.394$; helix angle $\beta=19.548^{\circ}$; elastic deformation $\delta=0.0025 \mathrm{in}$. The other design parameters are the same as those mentioned in section 2.5 .

\subsection{Recommended Parameters of Rack-Cutter Normal Profiles}

The normal profiles of mating rack-cutters are shown in figure 2.7.1 (see also fig. 2.1.2(a)); $Q$ is the point of tangency of the profiles; $P$ relates to the pitch line (the instantaneous axis of rotation); $l_{i}=|\overline{Q P}|(i=c, t), \alpha_{n}$ is the normal pressure angle; $C_{2}$ and $C_{1}$ are the centers of curvature at point $Q$ of the gear and pinion profiles. The choice and determination of design parameters shown in figure 2.7.1 are based on the following considerations:

1. The normal pressure angle $\alpha_{n}$ is chosen as $25^{\circ}$ to $30^{\circ}$, which is in agreement with previous experience in designing the Novikov-Wildhaber helical gears. The increase in the pressure angle causes a higher sensitivity of the gearing to the error in the center distance and a larger value of the contact force. However, using a larger value of $x_{n}$ allows us to obtain more favorable relations between the curvatures of the gear tooth surfaces, thereby reducing the stresses.

2. The distance $l_{i}(i=c, t)$ is chosen as $l_{i}=\left(\cos \alpha_{n}\right) /\left(P_{n}\right)$, where $P_{n}$ is the normal diametral pitch.

3. The recommendation for $\rho_{c}=\left|Q C_{2}\right|$ and $\rho_{c}=\left|Q C_{2}\right|$ (fig. 2.7.1) are based on the following relations:

a. The radius of curvature of the gear rack-cutter must satisfy the inequality

$$
\rho_{t}<\frac{N_{2} \sin \alpha_{n}}{2 P_{n} \cos ^{3} \beta}+l_{t}
$$




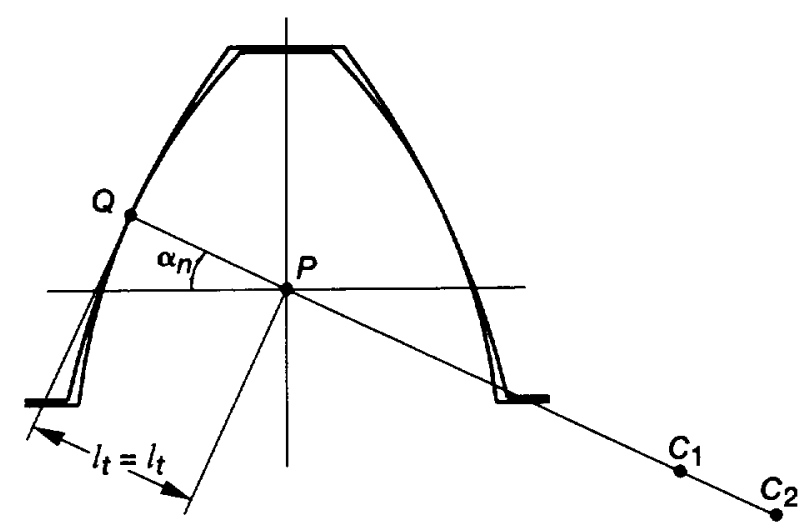

Figure 2.7.1. - Choice of design parameters for two rack-cutter normal profiles.

where $N_{2}$ is the number of gear teeth and $\beta$ is the helix angle.

Inequality (2.7.1) is derived by using the Euler-Savari equation that relates the curvatures of the transverse profiles of the gear rack-cutter and the gear. Inequality (2.7.1) is the condition of the gear normal profile to be concave in the neighborhood of the designated point $Q$.

b. We recommend that the radii of curvature $\rho_{t}$ and $\rho_{c}$ be

$$
\rho_{t}=\frac{6.35}{P_{n}}, \quad \rho_{c}=\frac{5.88}{P_{n}}
$$

to yield

$$
\frac{\rho_{t}}{\rho_{c}}=1.08
$$

Observing equations (2.7.2) and (2.7.3) allows us to keep the bearing contact shift caused by the change in the center distance in the working dimensions of the teeth.

c. The profiles of the rack-cutters are parabolic curves (see sec. 2.2). The parabolic coefficients $a_{c}$ and $a_{t}$ and the curvature radii are related as follows

$$
a_{c}=\frac{1}{2 \rho_{c}}, \quad a_{t}=\frac{1}{2 \rho_{t}}
$$

4. The values of the addendum and dedendum of the rack-cutter profiles must guarantee that the undercutting and pointing of generated teeth be avoided. Section 2.4 gives the results of an investigation of the avoidance of undercutting and pointing.

\subsection{Tooth Contact Analysis of Profile-Crowned Helical Gears}

The purpose of a TCA (tooth contact analysis) of profile-crowned helical gears is to prove that the meshing of such gears is accompanied by an unfavorable shape of the function of transmission errors that causes vibration and noise. Therefore it is necessary, as shown in section 4.0, to combine profile crowning with longitudinal crowning to transform the function of transmission errors to enable a reduction in the level of vibration and noise. Generating helical gears with profile and longitudinal crowning requires that existing technology be modified (see sec. 4.0 ). 


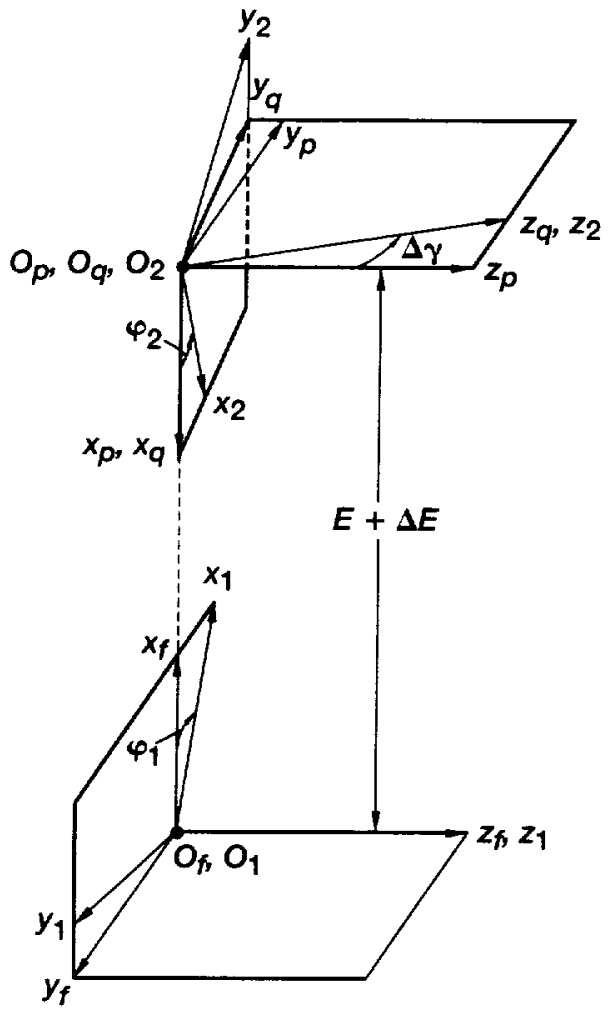

Figure 2.8.1.-Coordinate systems applied for TCA (tooth contact analysis).
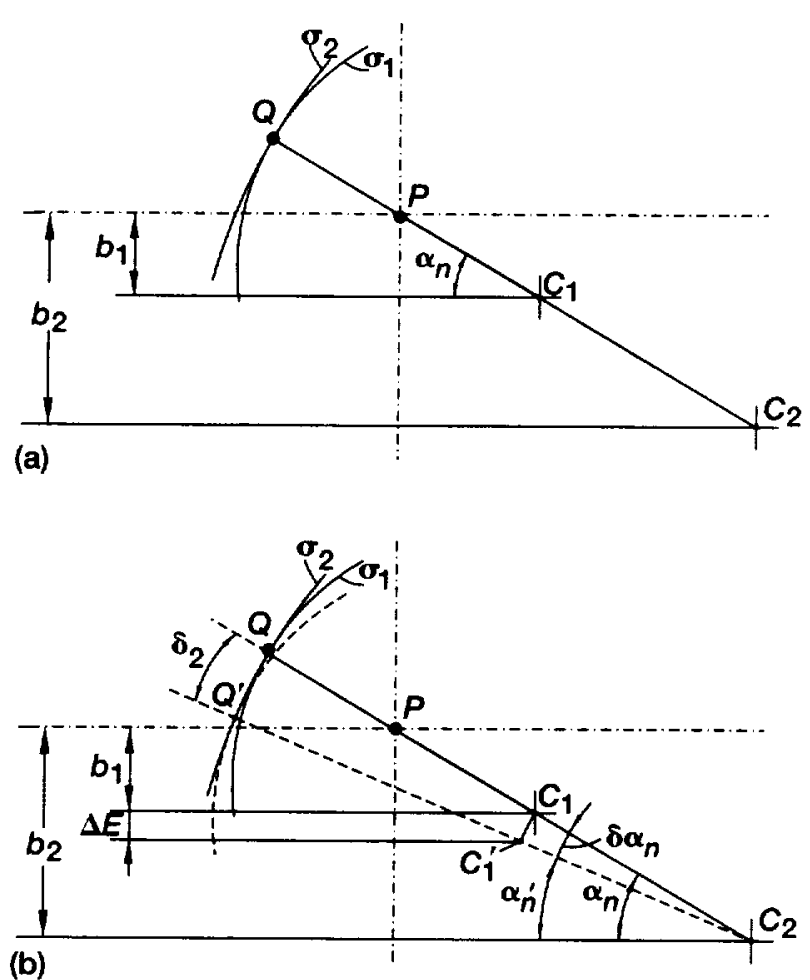

Figure 2.8.2.-Determination of change of normal pressure angle $\delta \alpha_{n}$ caused by change of center distance $\Delta E$. (a) Initial tangency of profiles $\sigma_{1}$ and $\sigma_{2}$ at point $Q$. (b) Tangency of $\sigma_{1}$ and $\sigma_{2}$ at point $Q^{\prime}$ that corresponds to error of center distance $\Delta E$.

Computer programs developed for TCA are based on the simulation of the continuous tangency of misaligned gear tooth surfaces. For this purpose, apply the following: (a) movable coordinate systems $S_{1}$ and $S_{2}$ rigidly connected to the pinion and the gear and (b) the basic fixed coordinate system $S_{f}$ where the meshing of the gears is considered (fig. 2.8.1). Coordinate systems $S_{p}$ and $S_{q}$ are auxiliary coordinate systems applied to simulate errors of alignment $\Delta E$, a change in the shortest center distance, and $\Delta \gamma$, a change in the shaft angle. In addition, the error in the lead angle $\Delta \lambda_{p i}(i=1,2)$ is also considered.

The TCA algorithm is based on the following procedure:

Step 1. - The pinion and gear tooth surfaces and their unit normals in coordinate systems $S_{1}$ and $S_{2}$ are initially represented as follows:

1. Surface $\Sigma_{1}$ and surface unit normal $\mathbf{n}_{1}$ are represented by vector functions $\mathbf{r}_{1}\left(u_{c}, \psi_{1}\right)$ and $\mathbf{n}_{1}\left(u_{c}, \psi_{1}\right)$.

2. Similarly, for surface $\Sigma_{2}$, we have vector functions $\mathbf{r}_{2}\left(u_{t}, \psi_{2}\right)$ and $\mathbf{n}_{2}\left(u_{t}, \psi_{2}\right)$.

Step 2. - Using coordinate transformation, represent $\Sigma_{1}, \mathbf{n}_{1}, \Sigma_{2}$, and $\mathbf{n}_{2}$ in $S_{f}$ The continuous tangency of $\Sigma_{1}$ and $\Sigma_{2}$ is represented by the vector equations

$$
\begin{gathered}
\mathbf{r}_{f}^{(1)}\left(u_{c}, \psi_{1}, \varphi_{1}, \Delta \lambda_{p 1}\right)=\mathbf{r}_{f}^{(2)}\left(u_{t}, \Psi_{2}, \varphi_{2}, \Delta \gamma, \Delta E\right) \\
\mathbf{n}_{f}^{(1)}\left(u_{c}, \psi_{1}, \varphi_{1}, \Delta \lambda_{p 1}\right)=\mathbf{n}_{f}^{(2)}\left(u_{t}, \psi_{2}, \varphi_{2}, \Delta \gamma\right)
\end{gathered}
$$


Errors of alignment $\Delta \gamma$ and $\Delta E$ are simulated by the proper installment of coordinate system $S_{q}$ with respect to $S_{f}$ (fig. 2.8.1). It is assumed that the error of the lead angle is considered for the pinion only. Angles $\varphi_{1}$ and $\varphi_{2}$ represent the rotation of pinion and gear being in mesh with each other.

Step 3.-Vector equation (2.8.1) is equivalent to three independent scalar equations, but (2.8.2) is only equivalent to two independent scalar equations since $\left|\mathbf{n}_{f}^{(1)}\right|=\left|\mathbf{n}_{f}^{(2)}\right|=1$. The system of vector equations (2.8.1) and (2.8.2) is equivalent to five independent scalar equations in six unknowns represented as

$$
f_{i}\left(u_{c}, \psi_{1}, \varphi_{1}, u_{t}, \psi_{2}, \varphi_{2}\right)=0
$$

One of the unknowns, for example $\varphi_{1}$, can be chosen as the input with the goal being to obtain the solution of the equations by functions

$$
u_{c}\left(\varphi_{1}\right), \psi_{1}\left(\varphi_{1}\right), u_{t}\left(\varphi_{1}\right), \psi_{2}\left(\varphi_{1}\right), \varphi_{2}\left(\varphi_{1}\right)
$$

This is an iterative process that requires at each iteration the first guess and the observation of the inequality (refs. 7 and 8).

$$
\frac{D\left(f_{1}, f_{2}, f_{3}, f_{4}, f_{5}\right)}{D\left(u_{c}, \psi_{1}, \varphi_{1}, u_{t}, \psi_{2}, \varphi_{2}\right)} \neq 0
$$

The iterative process is computerized by using the program DNEQNF (ref. 9). The output of TCA enables us to determine the function of transmission errors $\Delta \varphi_{2}\left(\varphi_{1}\right)$ and the shift of the bearing contact caused by misalignment.

Investigation shows that the most dominating error for the shift of bearing contact is the error of the center distance. The approach developed in this report is based on an investigation of the meshing of the normal profiles of mating rack-cutters.

Step 1.-Figure 2.8.2(a) shows that the normal profiles $\sigma_{1}$ and $\sigma_{2}$ of the rack-cutters are initially in tangency at point $Q$. The curvature radii are $\rho_{1}=Q C_{1}$ and $\rho_{2}=Q C_{2}$, and the location of the centers of curvature $C_{1}$ and $C_{2}$ with respect to $P$ is determined by $b_{1}$ and $b_{2}$.

Step 2. - Simulate the tangency of $\sigma_{1}$ and $\sigma_{2}$ when the center distance of the pinion and the gear is changed, imagining that $\sigma_{2}$ is held at rest and normal profile $\sigma_{1}$ is turned about $C_{2}$ through $\delta \alpha_{n}$ (fig. 2.8.2(b)). Then, center $C_{1}$ will take position $C_{1}^{\prime}$ and the normal profiles become in tangency at $Q^{\prime}$. The new pressure angle is $\alpha_{n}^{\prime}$, and the shift of the tangency point over $\sigma_{2}$ is

$$
\delta_{2}=\rho_{2} \delta \alpha_{n}, \quad \delta_{1}=\rho_{1} \delta \alpha_{n}
$$

It is easy to verify that

$$
\sin \alpha_{n}^{\prime}=\frac{b_{2}-b_{1}-\Delta E}{\rho_{2}-\rho_{1}}
$$

To reduce the shift of the point of contact over the profiles of the rack-cutters (and over the surfaces of the pinion and gear), it is necessary to increase the magnitude of $\left(\rho_{2}-\rho_{1}\right)$. The new design of helical gears developed in this report provides more room for such a solution without substantially affecting the contact stresses because the curvature radii $\rho_{2}$ and $\rho_{1}$ are substantially larger in comparison with the previous design of Novikov-Wildhaber gears.

Although the approach discussed for investigating the influence of $\Delta E_{1}$ is an approximate one, it provides a high precision that is confirmed by applying the developed TCA computer program. The change of center distance $\Delta E$ does not cause transmission errors.

Figures 2.8.3 and 2.8.4 graphically represent the influence of errors $\Delta \gamma$ and $\Delta \lambda_{p 1}$ on the transmission errors. The function of transmission errors becomes a discontinuous one and worsens the conditions for the transfer of meshing. 


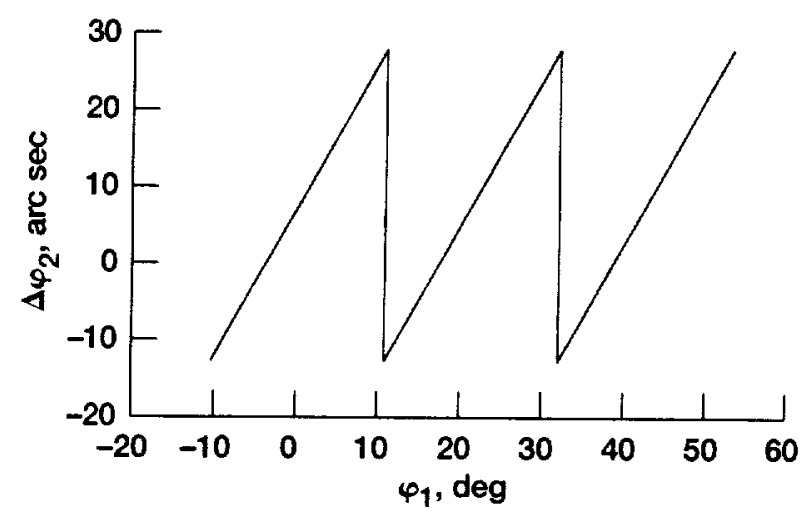

Figure 2.8.3.- Linear transmission error function when only profile crowning is applied; $\Delta \gamma=3$ arc min.

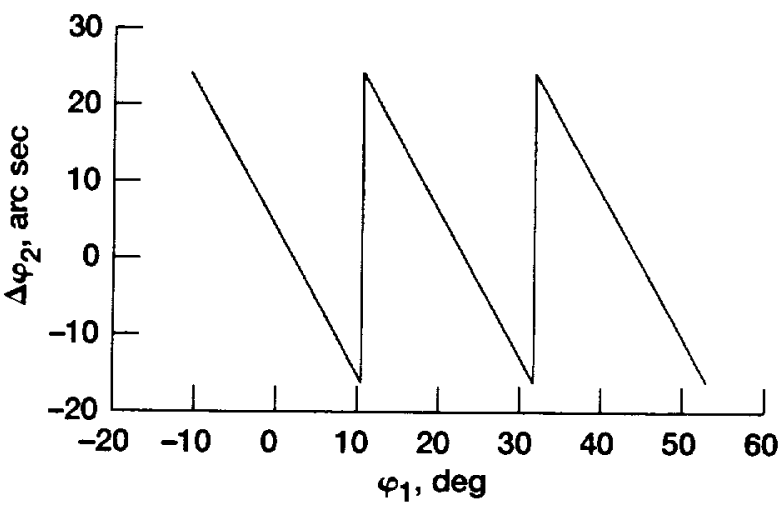

Figure 2.8.4.- Linear transmission error function when only profile crowning is applied; $\Delta \lambda=3$ arc min.

Gear vibration and noise become inevitable if only profile crowning is provided. This is the reason why combined crowning (in the profile and longitudinal directions) has been developed by the authors (see sec. 4.0).

\subsection{HELICAL PINION CONCAVE AND GEAR CONVEX PROFILE-CROWNED TOOTH SURFACES}

\subsection{Introduction}

Section 2.0 covers the proposed design of helical gears with a pinion convex-gear concave type of surface contact. We consider in this section helical gears with pinion concave-gear convex surface contact. Our investigation shows that the first type of contact is preferable (see secs. 3.3 and 3.4). However, for the purpose of completeness of the project, the pinion concave-gear convex contact of surfaces is covered in this section, in addition to the investigation accomplished in section 2.0 .

This investigation used the same design methodology as applied in section 2.0. The contents of this section cover the choice of design parameters based on the results of investigating undercutting and pointing, determining the contact ellipse, and comparing the results obtained with those obtained in section 2.0 .

\subsection{Preliminary Choice of Design Parameters of the Normal Section of Rack-Cutters}

As mentioned in section 2.0, two imaginary rigidly connected rack-cutters $\Sigma_{c}$ and $\Sigma_{t}$ are applied to generate the pinion and the gear. The normal sections of the rack-cutters are shown in figure 3.2.1. The radius of curvature $\left|\overline{O_{a} C_{c}}\right|$ of the pinion rack-cutter at point $Q$ must satisfy the inequality

$$
\left|\overline{O_{c} C_{c}}\right|=\rho_{c}<\frac{N_{1} \sin \alpha_{n}}{2 P_{n} \cos ^{3} \beta}+l_{c}
$$

The right side of the inequality represents the curvature radius of the rack-cutter that generates point $Q$ of the pinion profile of an infinitely large curvature radius. The derivation of inequality (3.2.1) is based on the application of the Euler-Savari equation.

Inequality (3.2.1) guarantees that the pinion transverse profile is concave only at point $Q$ but not at any point of the pinion profile. This is illustrated by the drawings of figure 3.2.2, which shows transverse profiles of the pinion and the rack-cutter. The pinion profile is concave-convex. However, choosing proper design parameters makes it possible to obtain a concave pinion profile in the whole area. An example of a set of such design parameters is

$$
\alpha_{n}=25^{\circ}, l_{c}=\frac{0.906}{P_{n}}, a_{c}=\frac{1}{2 \rho_{c}}=1.25 P_{n}, \quad a_{t}=\frac{1}{2 \rho_{t}}=1.389 P_{n}, \quad N_{p 1} \geq 16
$$




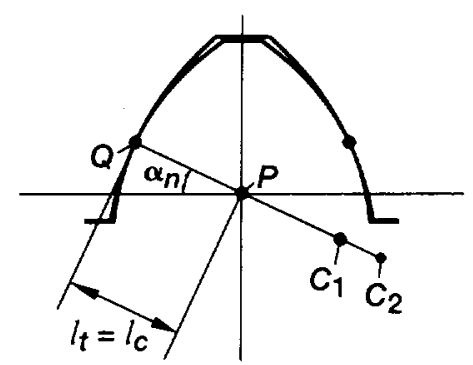

(a)

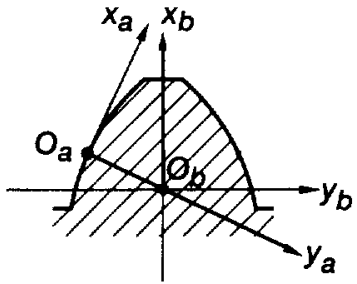

(b)

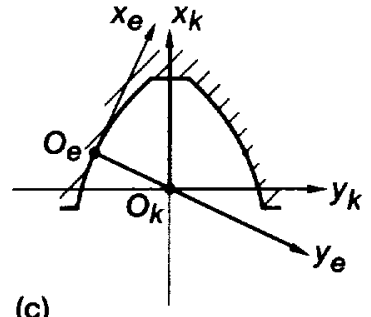

(c)

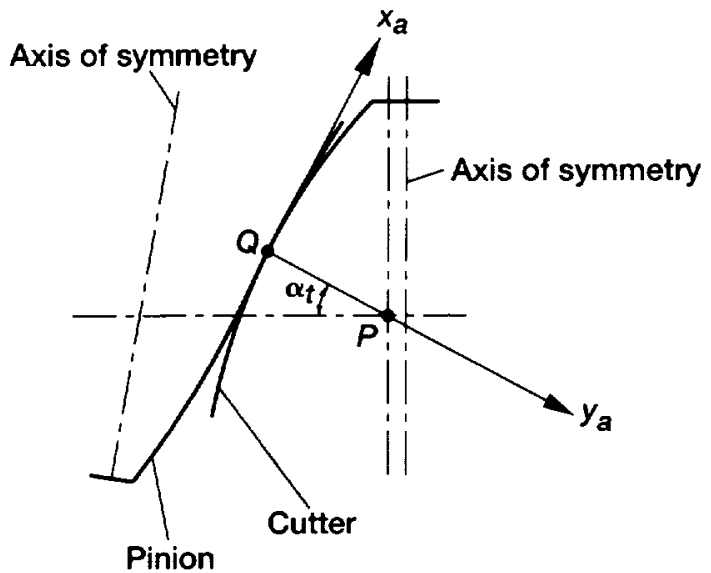

Figure 3.2.2.-Concave-convex pinion and convex rack-cutter transverse profiles.

Profiles of pinion concave an gear convex rackcutters. (b) Pinion rack-cutter. (c) Gear rack-cutter.

The limitations of the rack-cutter addendum and dedendum are determined in subsection 3.3.

\subsection{Avoidance of Undercutting and Pointing}

The developed approach is based on the same considerations that have been represented in section 2.4 . The final results of the concluded investigation are formulated as follows:

1. Undercutting by a rack-cutter may occur only for the generated convex profile gear and does not occur for the concave profile pinion. Undercutting may be avoided by limitations of the active part of the rack-cutter addendum. Function $h_{a} P_{n}\left(N_{2}\right)$ is represented in figure 3.3 .1 by curve 1 .

2. Pointing may occur for a gear with a small number of teeth. Curve 2 in figure 3.3.1 shows function $h_{d} P_{n}\left(N_{2}\right)$ where $h_{d}$ is the active part of the rack-cutter dedendum. The curve has been determined under the condition that the tooth thickness on the gear addendum circle be chosen as $t_{a}=\left(0.3 / P_{n}\right)$.

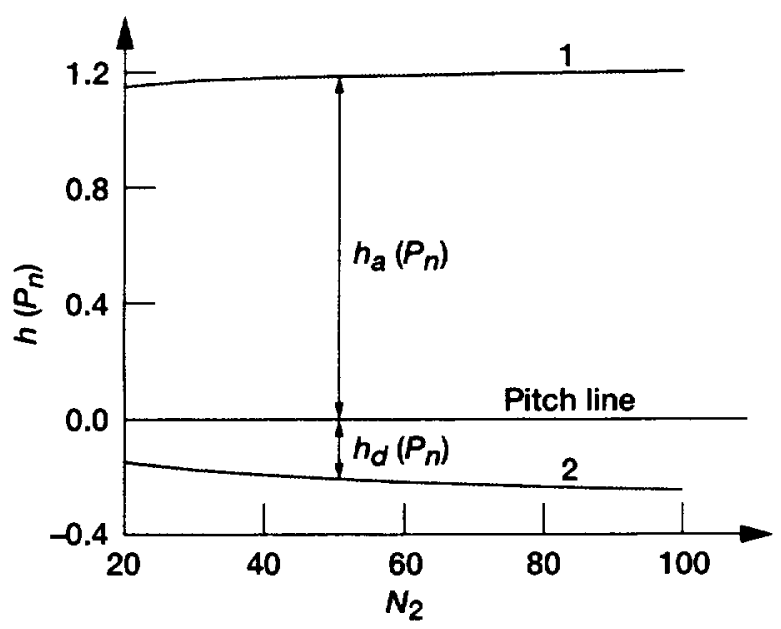

Figure 3.3.1.-Permissible dimensions of rack-cutter addendum $h_{a}$ and dedendum $h_{d}$. 

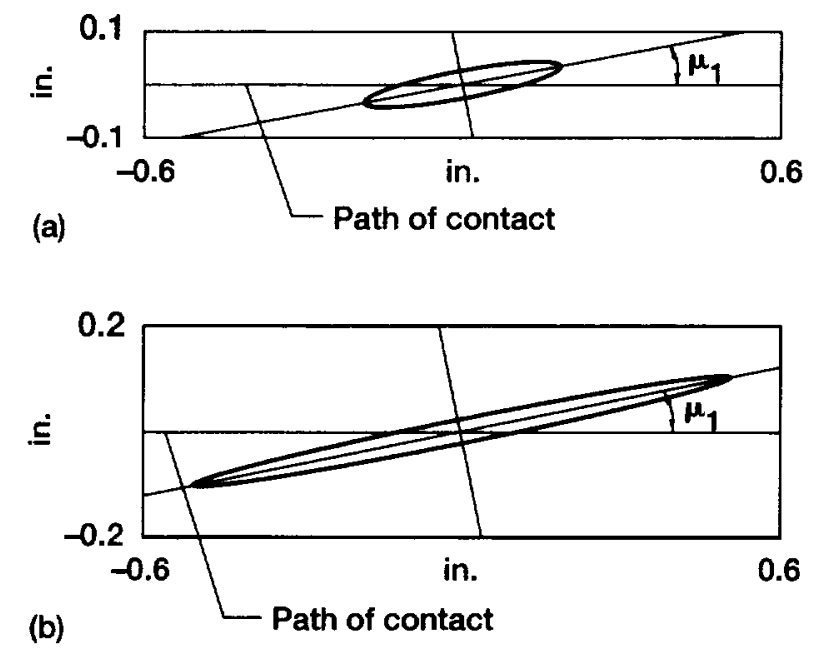

Figure 3.4.1.-Contact ellipse on pinion tooth surfaces. (a) Concave pinion tooth surface. (b) Convex pinion tooth surface.

The whole active tooth height for a gear with tooth number $N_{2} \geq 70$ is

$$
h=h_{a}+h_{d}=\frac{1.4}{P_{n}}
$$

Note that we have $h=\left(1.8 / P_{n}\right)$ in the case of the pinion convex-gear concave design that is obtained in section 2.0. The reduction of tooth height in the second version is a disadvantage of the pinion concave-gear convex design.

\subsection{Contact Ellipse}

Applying the approach discussed in 2.5 and 2.6, we have obtained the pinion and gear principal curvatures and directions and then have determined the dimensions and orientation of the instantaneous contact ellipse. The numerical computations have been obtained for both designed versions of helical gears and then were compared. The design parameters are the same as those applied in section 2.0. The obtained contact ellipses for both versions are shown in figure 3.4.1. It is evident that because of the decrease in the contact ellipse, the second version of the design cannot be recommended for application. The preferable design version is the pinion convex-gear concave type of surface contact.

\subsection{GENERATION OF DOUBLE-CROWNED PINION TOOTH SURFACE BY GRINDING DISK}

\subsection{Research Goals and Developed Approaches}

In sections 2.0 and 3.0 , profile crowning of pinion-gear tooth surfaces $\Sigma_{1}$ and $\Sigma_{2}$ was shown to be sufficient only for the localization of bearing contact. If crowning is accomplished in the profile direction only, misalignment of the gear drive will cause almost linear discontinuous functions of transmission errors (see curve $a$ in figure 1.8 and figs. 2.8.3 and 2.8.4). Then, high vibration of the gear drive becomes inevitable. To avoid such a defect, the authors have proposed the following methods for crowning:

1. Profile and longitudinal crowning is applied for pinion generation. Profile crowning is based on the mismatch of imaginary rack-cutter surfaces used to derive theoretical pinion-gear tooth surfaces. Longitudinal crowning of the pinion tooth surface is achieved by tool plunging. The tool may be (a) a grinding (or a cutting) disk or (b) a grinding worm (a hob). 
2. Only profile crowning is applied for gear generation, which is achieved by the mismatch of imaginary rackcutter surfaces as mentioned above.

This section covers the (1) generation of the pinion tooth surface by a plunging disk; (2) generation of the pinion tooth surface by a plunging worm; and (3) TCA for aligned and misaligned gear drives.

\subsection{Generation of Pinion Tooth Surface by Plunging Disk}

The proposed method for the generation is a modification of the existing method of form grinding and is based on the following considerations.

1. Consider initially that the pinion tooth surface $\Sigma_{1}$ is a helicoid determined as the envelope to the imaginary rack-cutter surface $\Sigma_{c}$. Surface $\Sigma_{1}$ is represented by vector function $\mathbf{r}_{1}\left(u_{c}, \psi_{1}\right)$ (see 2.2).

2. Then determine surface $\Sigma_{d}$ of the shaped disk, considering as given the pinion tooth surface $\Sigma_{1}$ (refs. 5 and 7 ). The axes of the pinion and the disk are crossed and the installment of the disk with respect to the pinion is shown in figure Al of appendix A. The disk surface $\Sigma_{d}$ is determined as the envelope to the family of pinion tooth surfaces. Figure 4.2.1 shows the cross-sectional profile of the grinding disk.

3. Consider at this stage that surface $\Sigma_{d}$ of the grinding disk is given and that the grinding disk performs a screw motion and is plunged with respect to the pinion. It is obvious that surface $\Sigma_{d}$ will generate a modified pinion tooth surface $\Sigma_{p}$ as the envelope to the family of surfaces $\Sigma_{d}$ of the grinding disk.

Detailed derivations are presented in appendixes $\mathrm{A}$ and $\mathrm{B}$.

The meshing and contact of pinion-gear tooth surfaces generated as proposed above have been tested by the TCA method, and the results are presented in section 5.5.

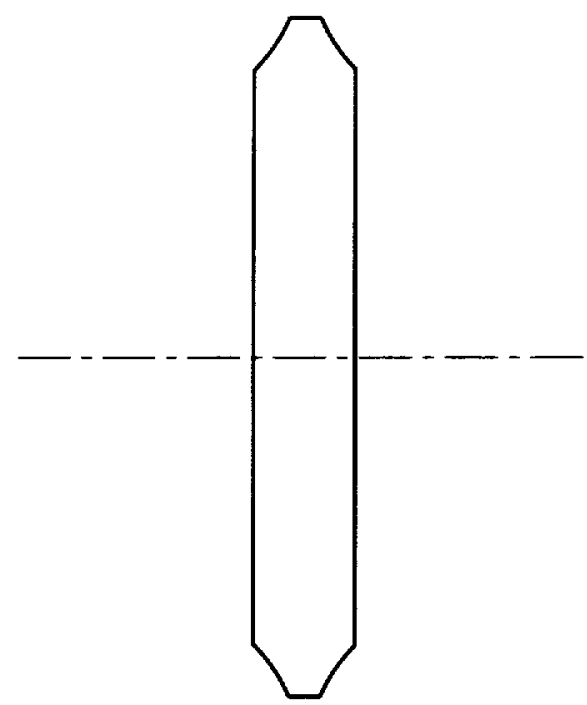

Figure 4.2.1.-Cross-sectional profile of grinding disk. 


\section{APPENDIX A \\ DETERMINATION OF SURFACE $\Sigma_{d}$ OF GENERATING DISK}

Introduction

Recall that the derivation of the pinion tooth surface generated by a plunging disk is divided into following stages (see 4.2):

1. Determination of disk surface $\Sigma_{d}\left(u_{d}, \theta_{d}\right)$ that is conjugated to the profile-crowned pinion tooth surface $\Sigma_{1}\left(u_{c}, \psi_{1}\right)$, which is considered a helicoid.

2. Determination of pinion tooth surface $\Sigma_{1}\left(u_{d}, \theta_{d}\right)$ generated by disk surface $\Sigma_{d}\left(u_{d}, \theta_{d}\right)$ that is plunged during the process generation.

Stages 1 and 2 of the derivations are presented in appendixes $A$ and $B$.

\section{Algorithm for Computation of Surface $\Sigma_{d}$}

The derivation of $\Sigma_{d}$ is based on the following procedure.

Step 1.-Apply three fixed coordinate systems $S_{1}, S_{d}$, and $S_{F}$. Coordinate systems $S_{1}$ and $S_{d}$ are rigidly connected to the pinion and the disk (figs. A.1 and 2). Coordinate system $S_{F}$ is an auxiliary fixed coordinate system. The drawings of figures A.1 and 2 illustrate the applied coordinate systems in orthogonal projections and in threedimensional space. The need for three coordinate systems and the reason they are considered as fixed is discussed in the following derivations.

Step 2. - The installment of the disk with respect to the pinion is defined by the shortest distance $E_{d}$ and the crossing angle $\gamma_{d}$. The shortest distance $E_{d}$ is determined by

$$
E_{d}=R_{o}+r_{1 d}
$$

where $R_{o}$ is the outer radius of the grinding disk and $r_{1 d}$ is the dedendum radius of the pinion.

The crossing angle $\gamma_{d}$ between the axes of rotation of the grinding disk and of the pinion is identical to the lead angle $\lambda_{p 1}$.

$\underline{\text { Step 3. }}$-The pinion tooth surface $\Sigma_{1}$ and its normal $N_{1}$ are represented in coordinate system $S_{1}$ by (sec. 2.2)

$$
\begin{aligned}
\mathbf{r}_{1} & =\mathbf{r}_{1}\left(u_{c}, \psi_{1}\right) \\
\mathbf{N}_{1} & =\mathbf{N}_{1}\left(u_{c}, \psi_{1}\right)
\end{aligned}
$$

where $\left(u_{c}, \psi_{1}\right)$ are the surface parameters.

Equations (A2) and (A3) are obtained for the case when axis $x_{1}$ is the tooth axis of symmetry (fig. A.3(a)). The grinding disk generates the pinion space and therefore we need the pinion tooth surface equations with axis $x_{1}$ as the axis of symmetry of space (fig. A.3(b)). This can be achieved by turning the pinion through angle

$$
\theta_{1}=\frac{\pi}{N_{1}}
$$

where $2 \pi / N_{1}$ is the pinion pitch angle. Using coordinate transformation, the pinion tooth surface and its normal may be represented now by vector equations

$$
\begin{aligned}
\mathbf{r}_{1} & =\mathbf{r}_{1}\left(u_{c}, \psi_{1}+\theta_{1}\right) \\
\mathbf{N}_{1} & =\mathbf{N}_{1}\left(u_{c}, \psi_{1}+\theta_{1}\right)
\end{aligned}
$$




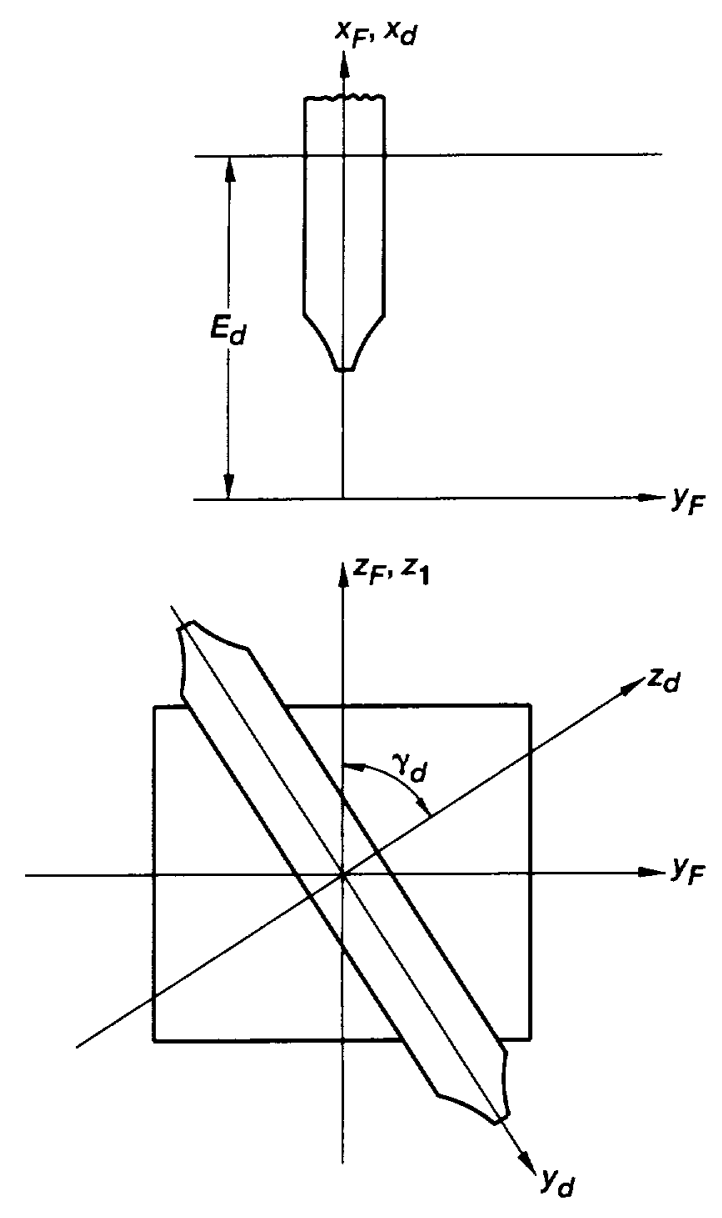

Figure A.1.-Installment of grinding disk.

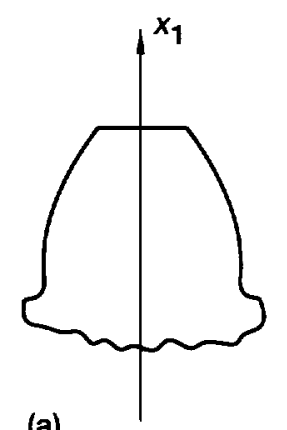

(a)

Figure A.3.-Orientation of axis of symmetry of (a) tooth and (b) space.

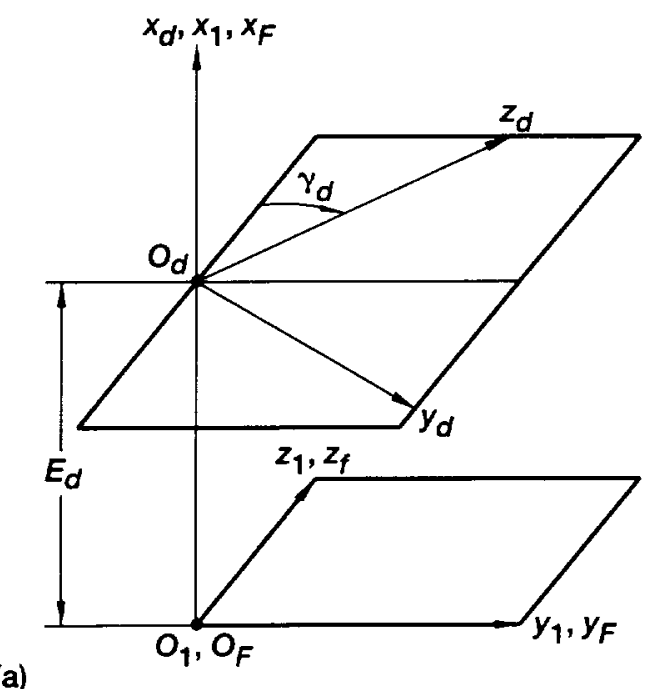

(b)

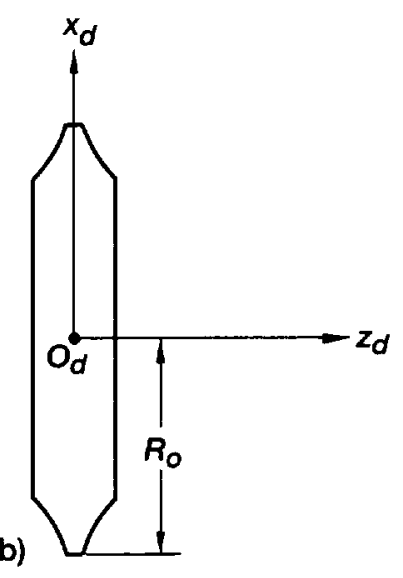

Figure A.2.-Coordinate systems applied for derivation of $\Sigma_{d^{*}}$ (a) Coordinate systems. (b) Disk axial section. 
Step 4. - Our next goal is to determine on surface $\Sigma_{1}$ the instantaneous line of tangency of surfaces $\Sigma_{1}$ and $\Sigma_{d}$. This can be accomplished considering that the normal $\mathbf{N}_{1}$ to $\Sigma_{1}$ at any point of tangency of $\Sigma_{1}$ and $\Sigma_{d}$ must pass through the grinding disk axis (refs. 5 and 7). This requirement, which follows from the condition that the disk surface be a surface of revolution, is represented by equations (refs. 5 and 7 )

$$
\frac{X_{1}-x_{1}\left(u_{c}, \psi_{1}\right)}{N_{x_{1}}\left(u_{c}, \psi_{1}\right)}=\frac{Y_{1}-y_{1}\left(u_{c}, \psi_{1}\right)}{N_{y_{1}}\left(u_{c}, \psi_{1}\right)}=\frac{Z_{1}-z_{1}\left(u_{c}, \psi_{1}\right)}{N_{z_{1}}\left(u_{c}, \psi_{1}\right)}
$$

where $\left(x_{1}, y_{1}, z_{1}\right)$ and $\left(N_{x_{1}}, N_{y_{1}}, N_{z_{1}}\right)$ are the point coordinates and the components of $\mathbf{N}_{1}$ at a current point on $\Sigma_{1}$; $X_{1}, Y_{1}, Z_{1}$ are the coordinates of the point of intersection of normal $\mathrm{N}_{1}$ to surface $\Sigma_{1}$ with the disk axis. Here, for the case when a left-hand pinion is generated (figs. A.4, 1, and 2), we have

$$
X_{1}=E_{d}, \quad \frac{Y_{1}}{Z_{1}}=\tan \gamma_{d}
$$

In the case of a left-hand helicoid, we have (refs. 5 and 7)

$$
y_{1} N_{x_{1}}-x_{1} N_{y_{1}}=-p N_{z_{1}}
$$

Equations (A7) to (A9) yield the relation

$$
f_{d_{1}}\left(u_{c}, \psi_{1}\right)=\left(E_{d}-x_{1}+p_{1} \cot \gamma_{d}\right) N_{z_{1}}-E_{d} \cot \gamma_{d} N_{y_{1}}+z_{1} N_{x_{1}}=0
$$

Equation $f_{d_{1}}=0$ may be called the equation of meshing of surfaces $\Sigma_{1}$ and $\Sigma_{d}$.

The line of tangency of $\Sigma_{1}$ and $\Sigma_{d}$ is determined by applying simultaneously equations (A5), (A6), and (A10). The same equations determine the line of tangency of $\Sigma_{1}$ and $\Sigma_{d}$ in coordinate system $S_{F}$ because coordinate systems $S_{1}$ and $S_{F}$ coincide with each other (figs. A.1 and 2).

Step 5. - The line of tangency between surfaces $\Sigma_{1}$ and $\Sigma_{d}$ is determined in coordinate system $S_{d}$ by

$$
\mathbf{r}_{d}\left(u_{c}, \psi_{1}\right)=\mathbf{M}_{d F} \mathbf{r}_{F}\left(u_{c}, \psi_{1}\right), \quad \mathbf{N}_{d}\left(u_{c}, \psi_{1}\right)=\mathbf{L}_{d F} \mathbf{N}_{F}\left(u_{c}, \psi_{1}\right), \quad f_{d F}\left(u_{c}, \psi_{1}\right),=0
$$

where $\mathbf{r}_{F}\left(u_{c}, \psi_{1}\right) \equiv \mathbf{r}_{1}\left(u_{c}, \psi_{1}+\theta_{1}\right), \mathbf{N}_{F}\left(u_{c}, \psi_{1}\right) \equiv \mathbf{N}_{1}\left(u_{c}, \psi_{1}+\theta_{1}\right), f_{d F}\left(u_{c}, \psi_{1}\right) \equiv f_{d 1}\left(u_{c}, \psi_{1}\right)=0$.

Step 6. - The next goal is to determine the axial profile of the grinding surface $\Sigma_{d}$. Equations (A11) enable us to determine the coordinates of the current point $M$ of the line of tangency of $\Sigma_{d}$ and $\Sigma_{1}$ by equations

$$
x_{d}=x_{d}\left(u_{c}, \psi_{1}\right), \quad y_{d}=y_{d}\left(u_{c}, \psi_{1}\right), z_{d}=z_{d}\left(u_{c}, \psi_{1}\right), \quad f_{d F}\left(u_{c}, \psi_{1}\right)=0
$$

The axial profile of the grinding disk (fig. A.5) is determined by

$$
x_{d}^{(M)}=-\rho=-\left(x_{d}^{2}, y_{d}^{2}\right)^{0.5}, \quad z_{d}^{(M)}\left(u_{c}, \psi_{1}\right), \quad f_{d F}\left(u_{c}, \psi_{1}\right)=0
$$

The disk surface $\Sigma_{d}\left(u_{d}, \theta_{d}\right)$ may be formed by rotating the axial profile about the disk axis; parameter $\theta_{d}$ is the current angle of rotation of the axial profile. 


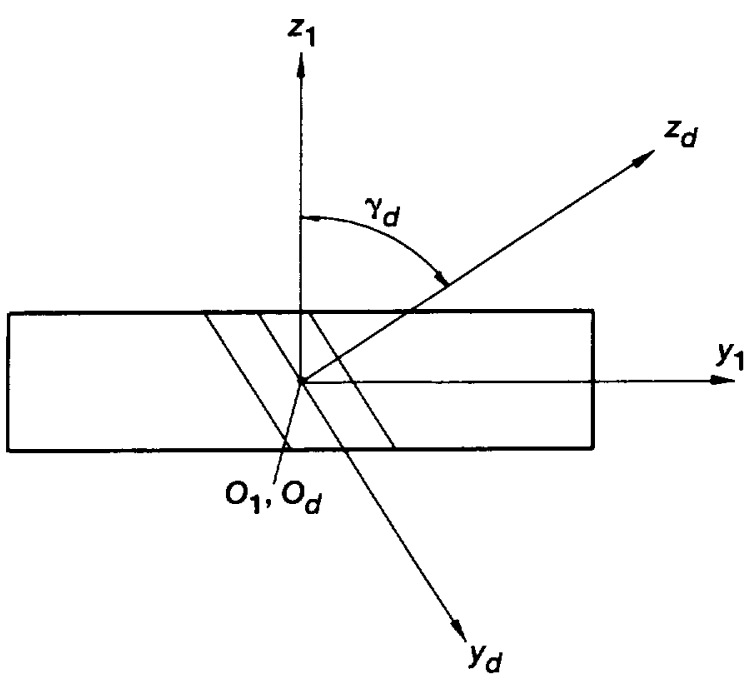

Figure A.4.- Orientation of coordinate system $S_{d}$ with respect to $S_{1}$ or a left-hand pinion.

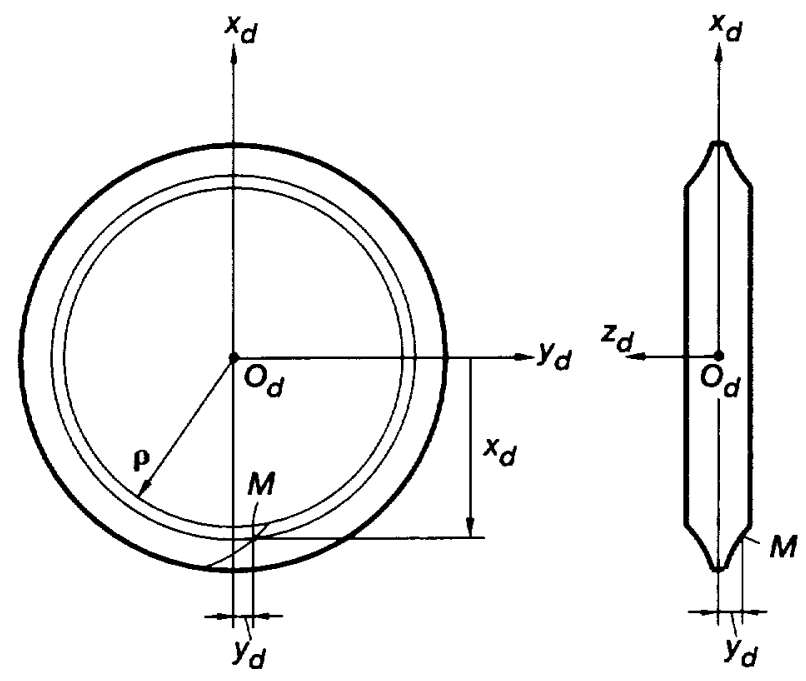

Figure A.5.-Derivation of axial profile of grinding disk.

\section{Determination of Crossing Angle $\gamma_{d}$}

The determination of crossing angle $\gamma_{d}$ must satisfy the following requirements:

1. The line of tangency between $\Sigma_{d}$ and $\Sigma_{1}$ has to contain point $Q$ of the tangency of the pinion rack-cutter and the pinion tooth surface in their common normal section (fig. 4.1.1). Recall that point $Q$ is simultaneously the point of tangency of pinion-gear tooth surfaces for an aligned gear drive. This requirement is satisfied with pinion surface parameter $u_{c}=0$.

2. The normal to the pinion tooth surface should not only intersect the axis of the grinding disk but pass through a point of the pinion tooth surface $\Sigma_{1}$ that belongs to plane $z_{F}=0$.

These two requirements yield the following equations in two unknowns, $\psi_{1}$ and $\gamma_{\mathrm{d}}$ :

$$
\begin{gathered}
z_{F}^{(1)}\left(u_{c}, \psi_{1}\right) \equiv z_{F}^{(1)}\left(0, \psi_{1}\right)=0 \\
f_{d 1}\left(u_{c}, \psi_{1}\right) \equiv f_{d 1}\left(0, \psi_{1}\right)=\left[E_{d}-x_{F}^{(1)}\left(0, \psi_{1}\right)+p_{1} \cot \gamma_{d}\right] N_{z F}^{(1)}\left(0, \psi_{1}\right)-E_{d} \cot \gamma_{d} N_{y F}^{(1)}\left(0, \psi_{1}\right)=0
\end{gathered}
$$

where (A15) is the transformed equation of meshing.

The final equation for the determination of $\gamma_{d}$ is

$$
\cot \gamma_{d}=\frac{\left[E_{d}-x_{F}^{(1)}\left(0, \psi_{1}^{*}\right)\right] N_{s F}^{(1)}\left(0, \psi_{1}^{*}\right)}{E_{d} N_{y F}^{(1)}\left(0, \psi_{1}^{*}\right)-p_{1} N_{s F}^{(1)}\left(0, \psi_{1}^{*}\right)}
$$

where $\psi_{1}^{*}$ is determined from

$$
z_{F}^{(1)}\left(0, \psi_{1}^{*}\right)=0
$$




\section{APPENDIX B \\ COMPUTERIZED GENERATION OF PINION TOOTH SURFACE BY PLUNGING DISK}

We designate the pinion tooth surface generated by the plunging disk as $\Sigma_{1}\left(\psi_{1}, \psi_{p}\right)$ to differentiate it from surface $\Sigma_{1}\left(u_{c}, \psi_{1}\right)$. Recall that $\Sigma_{1}\left(u_{c}, \psi_{1}\right)$ is a profile-crowned pinion surface whereas $\Sigma_{1}\left(\psi_{1}, \psi_{p}\right)$ is the pinion surface crowned in the profile and longitudinal directions. The longitudinal crowning is provided by the plunge of the grinding disk.

For the derivation of $\Sigma_{1}\left(\Psi_{1}, \psi_{p}\right)$, we apply two coordinate systems: $S_{n}$, the fixed, and $S_{p}$, the movable, which is rigidly connected to the pinion (fig. B.1). The derivation of pinion surface $\Sigma_{1}\left(\psi_{1}, \psi_{p}\right)$ is based on the following considerations:

1. In appendix $A$, the line of tangency of profile-crowned pinion tooth surface $\Sigma_{1}\left(u_{c}, \psi_{1}\right)$ and the grinding disk surface $\Sigma_{d}$ has been determined. This line of tangency is represented in $S_{n}$ by

$$
\mathbf{r}_{n}^{(1)}=\mathbf{r}_{n}^{(1)}\left(u_{c}, \psi_{1}\right), \quad f_{d 1}\left(u_{c}, \psi_{1}\right)=0
$$

where $\mathbf{r}_{n}^{(1)}\left(u_{c}, \psi_{1}\right) \equiv \mathbf{r}_{1}\left(u_{c}, \psi_{1}+\theta_{1}\right)$ (see eq. (A5)), and $f_{d 1}=0$ is the equation of meshing represented by (A10).

2. Coordinate system $S_{n}$ and the grinding disk are held at rest while coordinate system $S_{p}$ performs a screw motion with the pinion and in addition is plunged with respect to $S_{n}$. The plunge is provided to the pinion instead of the disk. Figure B.1 shows the screw motion of a left-hand pinion and the components of this motion are (a) displacement $l=p_{1} \psi_{p}$, where $p_{1}$ is the pinion screw parameter, and (b) rotation through angle $\psi_{p}$. Additionally provided is the plunge displacement $\Delta E_{d}\left(\psi_{p}\right)$ of the pinion in the direction of the shortest distance where

$$
\Delta E_{d}\left(\psi_{p}\right)=a_{p d}\left[l\left(\psi_{p}\right)\right]^{2}=a_{p d}\left(p_{1} \psi_{p}\right)^{2}
$$

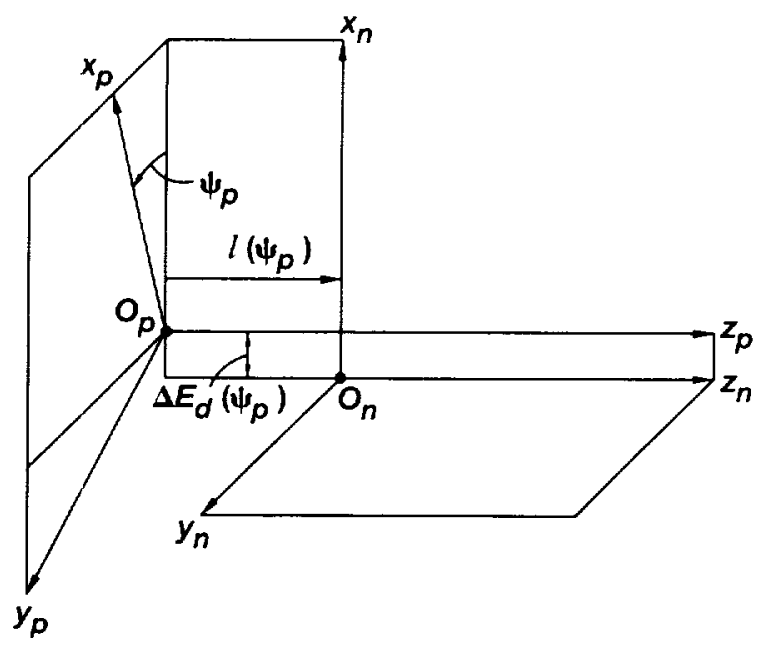

Figure B.1.-Coordinate systems applied for derivation of pinion surface $\Sigma_{1}$.

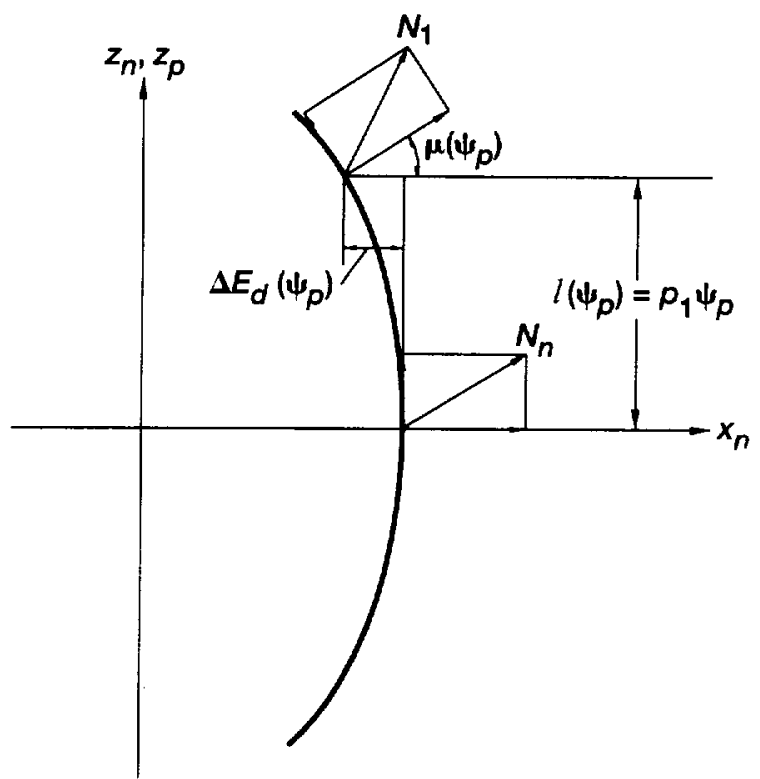

Figure B.2.-Determination of normal to generated pinion tooth surface $\Sigma_{1}$. 
The pinion-generated surface is determined as the family of spatial lines generated in the $S_{p}$ coordinate system while the pinion performs the motions described above. Surface $\Sigma_{1}$ is represented by

$$
\mathbf{r}_{1}\left(u_{c}, \psi_{1}, \psi_{p}\right)=\mathbf{M}_{p n}\left(\psi_{p}\right) \mathbf{r}_{n}\left(u_{c}, \psi_{1}\right), \quad f_{d n}\left(u_{c}, \psi_{1}\right)=0
$$

where $f_{d n}\left(u_{c}, \psi_{1}\right) \equiv f_{d F}\left(u_{c}, \psi_{1}\right) \equiv f_{d 1}\left(u_{c}, \psi_{1}\right)=0$.

Here (fig. B.1)

$$
\mathbf{M}_{p n}\left(\psi_{p}\right)=\left[\begin{array}{cccc}
\cos \psi_{p} & \sin \psi_{p} & 0 & -\Delta E_{d}\left(\psi_{p}\right) \cos \psi_{p} \\
-\sin \psi_{p} & \cos \psi_{p} & 0 & \Delta E_{d}\left(\psi_{p}\right) \sin \psi_{p} \\
0 & 0 & 1 & 1\left(\psi_{p}\right) \\
0 & 0 & 0 & 1
\end{array}\right]
$$

Consider that surface $\Sigma_{1}$ is represented by vector function

$$
\mathbf{r}_{1}\left[u_{c}\left(\psi_{1}\right), \psi_{1}, \psi_{p}\right] \equiv \mathbf{r}_{1}\left(\psi_{1}, \psi_{p}\right)
$$

Function $u_{c}\left(\psi_{1}\right)$ can be determined using the equation of meshing $f_{d 1}=0$ (see eq. (A10)).

Then, determine the normal to $\Sigma_{1}\left(\psi_{1}, \psi_{p}\right)$

$$
\mathbf{N}_{1}\left(\psi_{1}, \psi_{p}\right)=\frac{\partial \mathbf{r}_{1}}{\partial \psi_{1}} \times \frac{\partial \mathbf{r}_{1}}{\partial \psi_{p}}
$$

where

$$
\frac{\partial \mathbf{r}_{1}}{\partial \psi_{1}}=\frac{\partial \mathbf{r}_{1}}{\partial u_{c}} \frac{\mathrm{d} u_{c}}{\mathrm{~d} \psi_{1}}+\frac{\partial \mathbf{r}_{1}}{\partial \psi_{1}}
$$

A simplified approach to the derivation of normal $N_{1}$ to surface $\Sigma_{1}\left(\psi_{1}, \psi_{p}\right)$ is based on the following considerations:

1. The initial position of the common normal $\mathbf{N}_{n}$ (fig. B2) to surface $\Sigma_{1}$ and the grinding disk surface $\Sigma_{d}$ is determined in coordinate system $S_{n}$ by equation (A3) as

$$
\mathbf{N}_{n}\left(u_{c}, \psi_{1}\right) \equiv \mathbf{N}_{1}\left(u_{c}, \psi_{1}\right), \quad f_{d n}\left(u_{c}, \psi_{1}\right)=0
$$

2. In the grinding process, the normal performs in coordinate system $S_{p}$ two motions simultaneously: (a) rotation about the pinion axis of angle $\psi_{p}$ (fig. B 1 ) and (b) rotation about the $y_{n}$-axis of angle $\mu\left(\psi_{p}\right)$ (fig. B2) determined by

$$
\tan \left[\mu\left(\psi_{p}\right)\right]=\frac{\mathrm{d}\left[\Delta E_{d}\left(\psi_{p}\right)\right]}{\mathrm{d}\left[l\left(\psi_{p}\right)\right]}=2 a_{p d} p_{1} \psi_{p}
$$


Then normal $\mathbf{N}_{1}$ can be determined as

$$
\mathbf{N}_{1}\left(u_{c}, \psi_{1}, \Psi_{p}\right)=\mathbf{L}_{p n}\left(\psi_{p}\right) \mathbf{L}_{n}\left[\mu\left(\Psi_{p}\right)\right] \mathbf{N}_{n}\left(u_{C}, \psi_{1}\right)
$$

where $\mathbf{L}_{p n}\left(\Psi_{p}\right)$ is the $3 \times 3$ submatrix of matrix $\mathbf{M}_{p n}\left(\Psi_{p}\right)$ (see eq. (B4)) and $\mathbf{L}_{n}$ can be determined as

$$
\mathbf{L}_{n}\left[\mu\left(\psi_{p}\right)\right]=\left[\begin{array}{ccc}
\cos \mu & 0 & -\sin \mu \\
0 & 1 & 0 \\
\sin \mu & 0 & \cos \mu
\end{array}\right]
$$

Parameters $u_{C}$ and $\psi_{1}$ are related by the equation of meshing (A10). 


\subsection{GENERATION OF PINION TOOTH SURFACE BY PLUNGING OF GRINDING WORM}

\subsection{Introduction}

The generation of a pinion (gear) by a grinding worm (hob) must be considered as a two-parameter enveloping process in which the related motions of the worm and the workpiece are represented by two independent groups. One of these two groups includes the related rotations of the worm and the workpiece. The other group is referred to as the feed motion provided in the direction of the workpiece.

In our case, the workpiece is the pinion and the longitudinal crowning is provided by the plunging of the worm with respect to the pinion in the direction of the shortest distance.

\subsection{Installment, Related Motions, and Applied Coordinate Systems}

Derivation of crossing angle.-Consider that a left-hand pinion is generated by a left-hand hob (grinding worm). It is more favorable for cutting conditions if the pinion and hob helices are in the same direction. The drawings of figure 5.2.1 show a left-hand hob (fig. 5.2.1(a)) and its installment with respect to a left-hand pinion (fig. 5.2.1(b)). It can be easily verified from the drawings that the crossing angle $\gamma_{w}$ is determined as

$$
\gamma_{w^{\prime}}=90^{\circ}-\beta_{p}-\lambda_{w^{\prime}}
$$

where $\beta_{p}$ is the pinion helix angle and $\lambda_{w^{\prime}}$ is the worm lead angle.

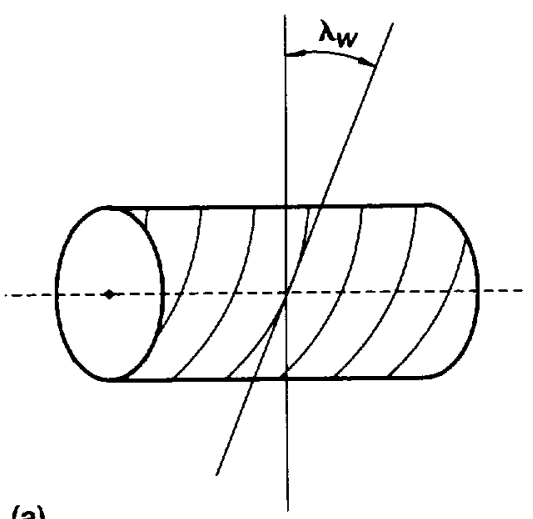

(a)

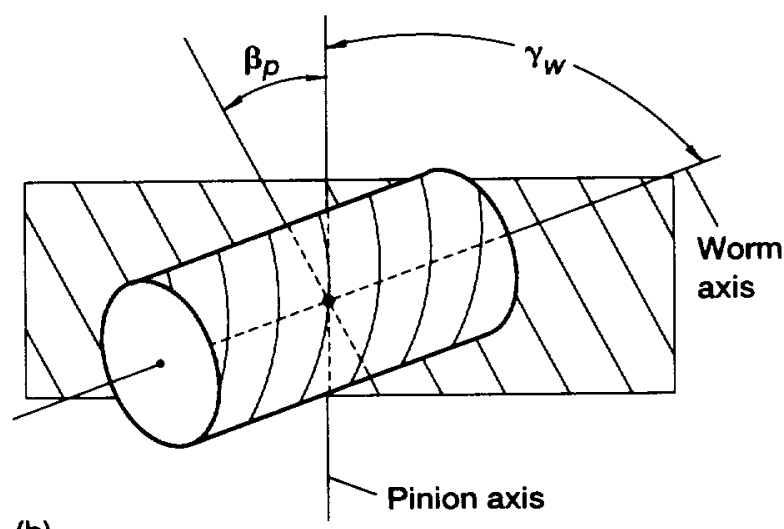

(b)

Figure 5.2.1.-Derivation of crossing angle $\gamma_{w}$ between pinion and worm axes. (a) Left-hand worm with lead angle $\lambda_{w}$. (b) Installment of worm with respect to pinion.

Related motions performed during feed motion.-Figure 5.2.2 shows two positions of the worm axis when the feed motion along the pinion axis is provided. The relative motion of the worm surface with respect to the pinion surface during the feed motion must be provided along the pinion screw surface, and therefore an additional translation motion of the worm (perpendicular to the pinion axis) determined as $l_{w^{*}} \tan \beta_{p}$ must be provided.

Axial worm translational motion.-A conventional method for generating spur and helical gears by a worm is based on the requirement that the piecework and the worm perform related rotations about their axes. However, for the purpose of simplifying the derivations, the rotation of the worm can be substituted by its translational motion in the axial direction. The following derivations are based on the consideration that the worm and the pinion being generated are simultaneously in mesh with the same rack that has generated the pinion.

Figure 5.2.3 shows the velocity polygon during the meshing process of the worm and the rack. The axial displacement of the worm may be represented in two components: $s_{k^{\prime}} \cos \lambda_{k^{\prime}}$ and $s_{k^{\prime}} \sin \lambda_{k^{\prime}}$ that are directed perpendicular to the tangent $t-t$ to the helix and along the helix. Similarly, the displacement of the rack may be represented 


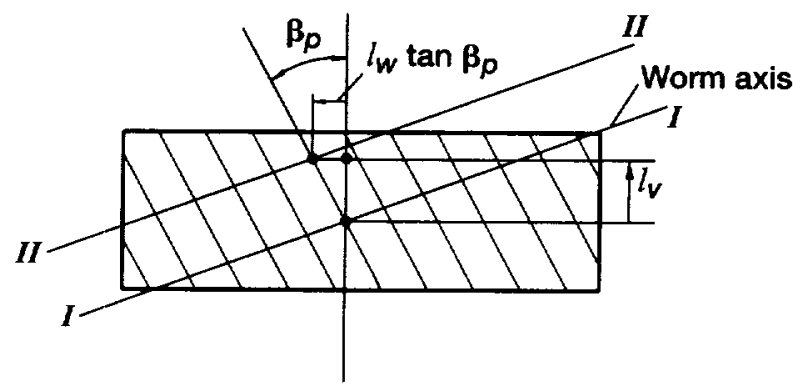

Figure 5.2.2.-Components of translational motion of worm performed during feed motion.

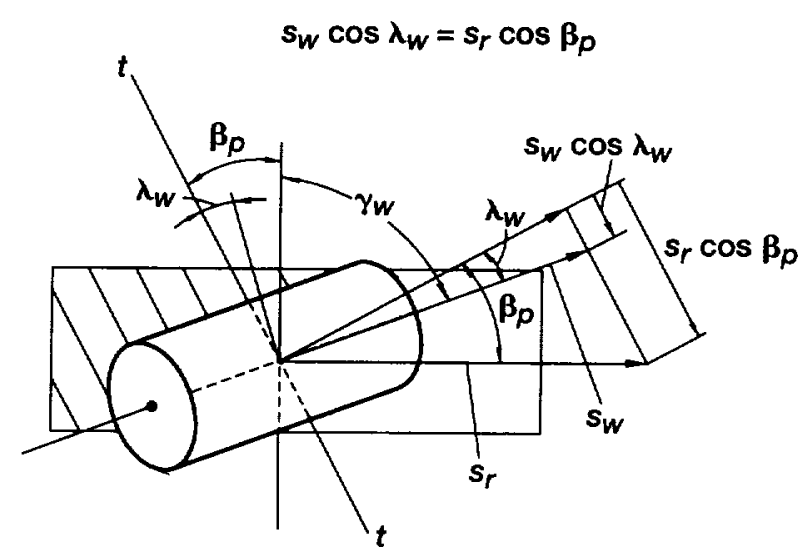

Figure 5.2.3.-Derivation of relations between axial displacement of worm and angle of rotation of pinion.

in normal and tangential components $s_{r} \cos \beta_{p}$ and $s_{r} \sin \beta_{p}$. The normal components of the worm and rack displacements must be equal. Therefore, we have

$$
s_{w^{\prime}} \cos \lambda_{u^{\prime}}=s_{r} \cos \beta_{p}
$$

The tangential components $s_{w} \sin \lambda_{w}$ and $s_{r} \sin \beta_{p}$ are not equal, which indicates sliding of the worm and rack surfaces. The displacement $s_{r}$, of the rack and the angle of pinion rotation are related by

$$
s_{r}=r_{p} \Psi_{p}
$$
rotation

Finally, we obtain the following relations between the axial displacement $s_{w}$ of the worm and the angle of gear

$$
s_{\mathcal{W}^{\prime}}=\frac{r_{p} \cos \beta_{p}}{\cos \lambda_{w^{\prime}}} \Psi_{p}=m \Psi_{p}
$$

Applied coordinate systems.-Movable coordinate systems $S_{\mathrm{u}}$ and $S_{p}$ are rigidly connected to the worm and the pinion; $S_{m}$ is the fixed coordinate system (fig. 5.2.4). Pinion axis $z_{p}$ coincides with the $z_{m}$-axis. The pinion and worm axes form the crossing angle $\gamma_{k}$. Planes $\Pi$ and $x_{m}=0$ are parallel to the plane that is tangent to the pitch cylinders of the pinion, and the gear worm axis $z_{w}$, lies in plane $\Pi$. The shortest distance between planes $\Pi$ and $x_{m}=0$ is varied in the generation process because of the plunging of the worm with respect to the pinion. The location of origin $O_{u^{\prime}}$ of coordinate system $S_{k}$ is represented in plane $\Pi$ by vectors $\overline{A B}$ and $\overline{B O_{w}}$, where

$$
\begin{gathered}
\overline{A B}=-l_{w^{\prime}} \tan \beta_{p} \mathrm{j}_{m}+l_{w^{\prime}} \mathrm{k}_{m} \\
\overline{B O_{w^{\prime}}}=s_{w^{\prime}}\left(\sin \gamma_{w^{\prime}} \mathrm{j}_{m}+\cos \gamma_{w} \mathrm{k}_{m}\right)
\end{gathered}
$$

\subsection{Generation of Pinion Surface $\Sigma_{p}$}

Worm surface. - The worm surface is determined as the envelope to the family of rack-cutter surfaces as discussed in section 2.0. The worm design parameters are

1. The normal diametral pitch, the same as the pinion normal diametral pitch 


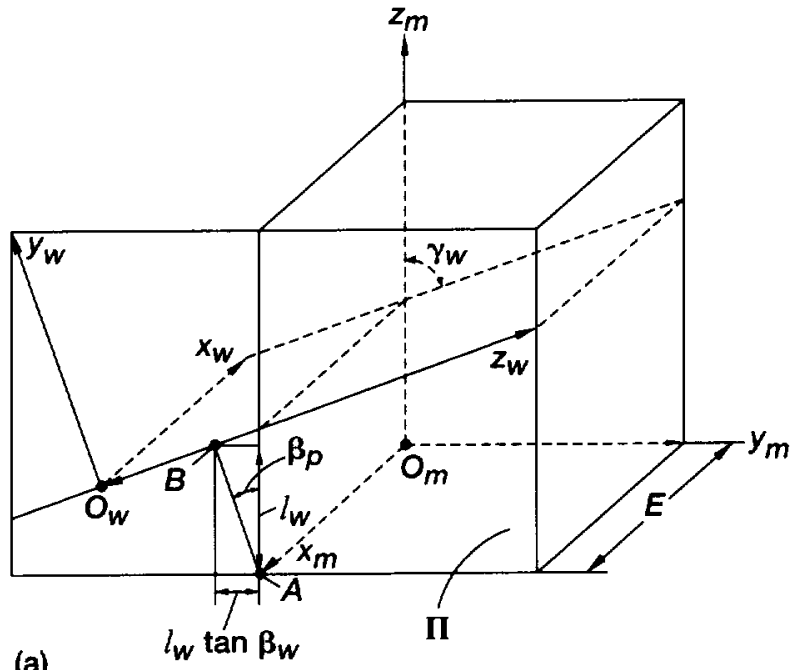

(a)

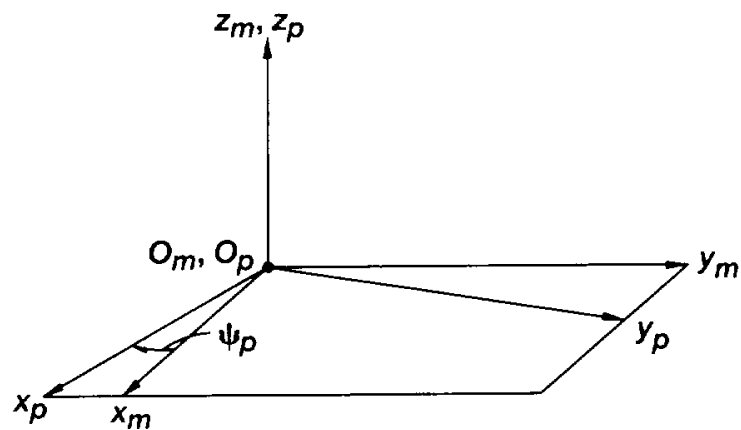

(b)

Figure 5.2.4.-Coordinate systems applied for generation of pinion by worm. (a) $S_{m}$ and $S_{w}$. (b) $S_{m}$ and $S_{p}$.

2. The worm thread number $N_{\mathrm{H}^{\prime}}$ (usually, $N_{\mathrm{H}^{\prime}}=1$ )

3. The worm pitch radius $r_{p w}$.

The worm lead angle is determined as

$$
\lambda_{w^{\prime}}=\arcsin \left(\frac{N_{u^{\prime}}}{2 P_{n} r_{p w^{\prime}}}\right)
$$

The worm surface is represented by vector function $\mathbf{r}_{k^{\prime}}\left(u_{w^{\prime}{ }^{\prime}}\right)$, which is similar to vector function $\mathbf{r}_{1}\left(u_{c}, \psi_{1}\right)$ (see eq. (2.2.6)).

Parameters of two-enveloping generation of pinion.-Surface $\Sigma_{p}$ is the envelope to the two-parameter family of worm surfaces $\Sigma_{n}$, which is (the family) represented in $S_{p}$. The independent parameters of motion are (1) the angle of pinion rotation $\psi_{p}$ and (2) the worm displacement $l_{n}$ in feed motion. The worm axial displacement $s_{k}$ depends on parameter $\psi_{p}$ and other worm displacements depend on parameter $l_{w}$.

The tangential displacement of the worm determined as $(\overline{A B}) \mathbf{j}_{m}$ substitutes an additional angle of pinion rotation $\Delta \psi_{p}^{*}$ that occurs in the real machine process and is determined as

$$
\Delta \Psi_{p}^{*}=\frac{l_{\mathrm{M}} \tan \beta}{r_{p}}
$$

The shortest distance $E$ between the worm and pinion axis is

$$
E\left(l_{w}\right)=E_{o}-a_{l} l_{w}^{2}
$$

The axial worm displacement $s_{w}$ is a function of parameter $\Psi_{p}$ (see eq. (5.2.4)).

Generation of worm family surfaces. - The family of worm thread surfaces is represented in $S_{p}$ by matrix equation

$$
\mathbf{R}_{p}\left(u_{w}, \Psi_{w}, l_{w}, \psi_{p}\right)+\mathbf{M}_{p m} \mathbf{M}_{m u}, \mathbf{r}_{w}\left(u_{w}, \psi_{w}\right)
$$


Here (fig. 5.2.4)

$$
\begin{aligned}
& \mathbf{M}_{p m}=\left[\begin{array}{cccc}
\cos \psi_{p} & -\sin \psi_{p} & 0 & 0 \\
\sin \psi_{p} & \cos \psi_{p} & 0 & 0 \\
0 & 0 & 1 & 0 \\
0 & 0 & 0 & 1
\end{array}\right] \\
& \mathbf{M}_{m w^{\prime}}=\left[\begin{array}{cccc}
-1 & 0 & 0 & \left(\overline{O_{w} O_{m}}\right) \cdot \mathbf{i}_{w^{\prime}} \\
0 & -\cos \gamma_{w^{\prime}} & \sin \gamma_{w^{\prime}} & \left(\overline{O_{w} O_{m}}\right) \cdot \mathbf{j}_{w^{\prime}} \\
0 & \sin \gamma_{k^{\prime}} & \cos \gamma_{u^{\prime}} & \left(\overline{O_{w^{\prime}} O_{m}}\right) \cdot \mathbf{k}_{\mathrm{u}^{\prime}} \\
0 & 0 & 0 & 1
\end{array}\right] \\
& \left.\begin{array}{l}
\left(\overline{O_{w^{\prime}} O_{m}}\right) \cdot \mathbf{i}_{w^{\prime}}=E_{o}-a_{l} l_{w^{\prime}}^{2}=E_{x m} \\
\left(\overline{O_{w^{\prime}} O_{m}}\right) \cdot \mathbf{j}_{w^{\prime}}=-l_{w^{\prime}} \tan \beta-m \psi_{p} \sin \gamma_{w^{\prime}}=E_{y m} \\
\left(\overline{O_{w} O_{m}}\right) \cdot \mathbf{k}_{w^{\prime}}=l_{w^{\prime}}-m \psi_{p} \cos \gamma_{w^{\prime}}=E_{z m}
\end{array}\right\}
\end{aligned}
$$

Equations of meshing of worm and pinion tooth surfaces.-In the two-parameter enveloping process, apply two equations of meshing (refs. 5 and 7):

$$
\begin{aligned}
& \mathbf{N}_{m}^{\left(w^{\prime}\right)} \cdot \mathbf{v}_{m}^{\left(u p, \psi_{p}\right)}=F_{1}\left(u_{w^{\prime}}, \psi_{w^{\prime}}, \Psi_{p}, l_{w^{\prime}}\right) \\
& \mathbf{N}_{m}^{\left(w^{\prime}\right)} \cdot \mathbf{v}_{m}^{\left(n \cdot p, l_{w}\right)}=F_{2}\left(u_{w^{\prime}}, \Psi_{w^{\prime}}, \psi_{p}, l_{w^{\prime}}\right)
\end{aligned}
$$

where subscript $m$ indicates that vectors in equations (5.3.8) and (5.3.9) are represented in coordinate system $S_{m}$; vector $\mathbf{N}_{m}^{\left(w^{\prime}\right)}$ represents the normal to the worm surface; vector $\mathbf{v}_{m}^{\left(w p, \psi_{p}\right)}$ represents the relative velocity in the motion when parameter $\psi_{p}$ is varied and parameter $l_{w^{\prime}}$ is fixed. Similarly, $\left.\mathbf{v}_{m}^{\left(w p, l_{w}\right.}\right)_{\text {is }}$ the relative velocity determined for the conditions when $l_{w}$, is the variable parameter and $\psi_{p}$ is fixed.

Equations (5.3.4), (5.3.8), and (5.3.9) represent the pinion tooth surface by four related parameters. The derivation of relative velocities $v_{m}^{\left(w p . \psi_{p}\right)}$ and $v_{m}^{\left(w p, l_{m}\right)}$ represented in equations (5.3.7) and (5.3.8) is based on the following procedure:

Step 1: Represent the velocity $\mathbf{v}_{m}^{\left(w p, \psi_{p}\right)}$ as

$$
\mathbf{v}_{m}^{\left(w \cdot p, \psi_{p}\right)}=\mathbf{v}_{m}^{\left(w p, \psi_{p}\right)}-\mathbf{v}_{m}^{\left(p, \psi_{p}\right)}
$$

and then determine $\mathbf{v}_{m}^{\left(w, \psi_{p}\right)}$ and $\mathbf{v}_{m}^{\left(p, \psi_{p}\right)}$.

Step 2: Represent $\mathbf{v}_{m}^{\left(p, \psi_{p}\right)}$ by

$$
\mathbf{v}_{m}^{\left(p, \psi_{p}\right)}=\omega_{m}^{(p)} \times \mathbf{r}_{m}
$$


where

$$
\omega_{m}^{(p)}=\left[\begin{array}{lll}
0 & 0 & 1
\end{array}\right]^{T}
$$

taking $\left|\omega_{m}^{(p)}\right|=1 \mathrm{rad} / \mathrm{sec}$ and $\mathbf{r}_{m}$ is the $3 \times 1$ vector. The $4 \times 1$ vector $\mathbf{r}_{m}$ is determined by matrix equation (see eqs. (5.3.6) and (5.3.7))

$$
\mathbf{r}_{w^{\prime}}=\mathbf{M}_{m w^{\prime}} \mathbf{r}_{w^{\prime}}=\left[\begin{array}{c}
-x_{w^{\prime}}+E_{x m} \\
-y_{w^{\prime}} \cos \gamma_{w^{\prime}}+z_{w^{\prime}} \sin \gamma_{w^{\prime}}+E_{y m} \\
y_{w^{\prime}} \sin \gamma_{w^{\prime}}+z_{w^{\prime}} \cos \gamma_{w^{\prime}}+E_{z m} \\
1
\end{array}\right]
$$

Step 3: Vector $\mathbf{v}_{m}^{\left(w ; \psi_{p}\right)}$ is represented as (see eqs. (5.3.6))

$$
\mathbf{v}_{m}^{\left(w, \psi_{p}\right)}=\left[\begin{array}{lll}
0 & \frac{\partial E_{y m}}{\partial \psi_{p}} & \frac{\partial E_{z m}}{\partial \psi_{p}}
\end{array}\right]^{T}=\left[\begin{array}{lll}
0 & m \sin \gamma_{w^{\prime}} & m \cos \gamma_{m}
\end{array}\right]^{T}
$$

Step 4: Equations (5.3.10) to (5.3.14) yield the following expression for $\mathbf{v}_{m}^{\left(w p, \psi_{p}\right)}$ :

$$
\mathbf{v}_{m}^{\left(w p, \psi_{p}\right)}=\left[\begin{array}{c}
E_{y m}-y_{w^{\prime}} \cos \gamma_{w^{*}}+z_{w^{\prime}} \sin \gamma_{w^{\prime}} \\
m \sin \gamma_{w^{\prime}}-E_{x m}+x_{w} \\
m \cos \gamma_{w^{\prime}}
\end{array}\right]
$$

Step 5: The determination of velocity $\mathbf{v}_{m}^{(w p . l)}$ is based on the following considerations:

a.

$$
\mathbf{v}_{m}^{(n \cdot p, l)}=\mathbf{v}_{m}^{\left(w^{\prime}, l\right)}-\mathbf{v}_{m}^{(p, l)}=\mathbf{v}_{m}^{\left(w^{*}\right)}
$$

since $\mathbf{v}_{m}^{(p, l)}=0$ (the pinion is considered as being at rest when the feed motion is provided).

b.

$$
\mathbf{v}_{m}^{(w, l)}=\frac{\partial}{\partial l_{w^{\prime}}}\left(\overline{O_{w^{\prime}} O_{m}}\right)=\left[\begin{array}{lll}
-2 a_{l} l_{w^{\prime}}-\tan \beta & 1
\end{array}\right]^{T}
$$

Thus we found both of the relative velocities using the equations of meshing (5.3.8) and (5.3.9). The normal to the worm surface is determined by expression

$$
\mathbf{N}_{m}^{\left(w^{\prime}\right)}=\mathbf{L}_{m w^{\prime}} \cdot \mathbf{N}_{\mathrm{w}^{\prime}}\left(u_{\mathrm{H}^{\prime}}, \Psi_{\mathrm{w}^{\prime}}\right)
$$

where $\mathbf{L}_{m w}$ is the $3 \times 3$ submatrix of matrix $\mathbf{M}_{m w}$ (see eq. (5.3.6)). The normal to the worm surface does not depend on the relative motion parameters $\psi_{p}$ and $l_{w}$, because the worm performs only translative motion in the fixed coordinate system $S_{m}$. 


\subsection{Relations Between Tool Plunging and Parabolic Function of Transmission Errors}

Recall that tool plunging $\Delta s_{p l}$ and transmission errors $\Delta \varphi_{2}$ are represented by the following parabolic functions:

$$
\begin{gathered}
\Delta s_{p i}=a_{p l} l^{2} \\
\Delta \varphi_{2}\left(\varphi_{1}\right)=a_{\varphi} \varphi_{1}^{2}
\end{gathered}
$$

where $a_{p l}(1 /$ in. $)$ and $a_{\varphi}(1 / \mathrm{rad})$ are the parabolic coefficients.

Our investigation resulted in the following approximate equation that relates the parabolic coefficients:

$$
a_{\varphi}=\frac{a_{p l} a_{p r} \tan \alpha_{n} \sin \alpha_{n}}{a_{p l} m_{t}^{2} \cos ^{2} \beta+a_{p r} m_{w} \sin \alpha_{n} \tan ^{2} \beta} \frac{N_{1}^{2}}{2 P_{n} N_{2}}
$$

Here

$$
a_{p r}=a_{c}-a_{t}
$$

where $a_{c}$ and $a_{t}$ are the parabolic coefficients for the normal profiles of the rack-cutters (see sec. 2.0) and

$$
\begin{gathered}
m_{t}=1-2 a_{t} l_{t} \\
m_{w}=1-2 a_{p l} r_{p w}
\end{gathered}
$$

where $r_{p u}$ is the radius of the worm pitch cylinder.

Errors of alignment cause a shift $\Delta l$ of the bearing contact as shown in figure 5.4.1. The relation between the error of alignment $(\Delta \lambda$ or $\Delta \gamma)$ and the shift $(\Delta l)$ is represented by

$$
\Delta l=\frac{\Delta \lambda m_{w}}{2 a_{p l} \tan \alpha_{n} \cos \beta}
$$

An effective way to restore the central location of the bearing contact by the correction of the lead (or the lead angle) is by using equation (5.4.7). The advantage of this approach is that the correction of the lead angle will not cause additional transmission errors because a parabolic type of transmission error is predesigned. The reader should recall that a predesigned parabolic function of transmission errors absorbs the almost linear functions of the transmission errors caused by misalignment.

The accuracy of equation (5.4.3) was tested by a computerized simulation of meshing. Curves a and $b$ in figure 5.4.1 represent the parabolic functions of transmissions obtained by TCA and by equation (5.4.3). The simulation of meshing was accomplished for a helical gear drive with a pinion crowned in the profile and longitudinal directions. The following input data were used for the computation: $N_{1}=17, N_{2}=77, P_{n}=5$ in. $.^{-1}, \beta=19.95^{\circ}$, $\alpha_{n}=25^{\circ}, a_{c}=0.425 \mathrm{in}^{-1}, a_{t}=0.394 \mathrm{in}^{-1}, a_{p l}=0.00139 \mathrm{in}^{-1}, r_{p w}=3.82 \mathrm{in}$. The small difference between curves a and $\mathrm{b}$ confirms that equation (5.4.3) may provide sufficient accuracy for the magnitude of $a_{p l}$ when the magnitude of $a_{\varphi}$ is a given.

A simpler but less accurate equation may be applied as a substitute for equation (5.4.3). The derivation of the proposed equation is based on the following considerations:

Step 1. - We consider initially that the gear teeth are profile crowned, the path of contact is a helix, and the line of action is a straight line that is parallel to the gear axes. Figure 5.4.2(a) shows the transverse profiles in mesh after rotation from the initial position through angle $\varphi_{1}=\pi / N_{1}$ and displacements of the point of contact along the line of action on the distance $l=B / 2$, where $B$ is the working width of the gear (not shown in this figure). 


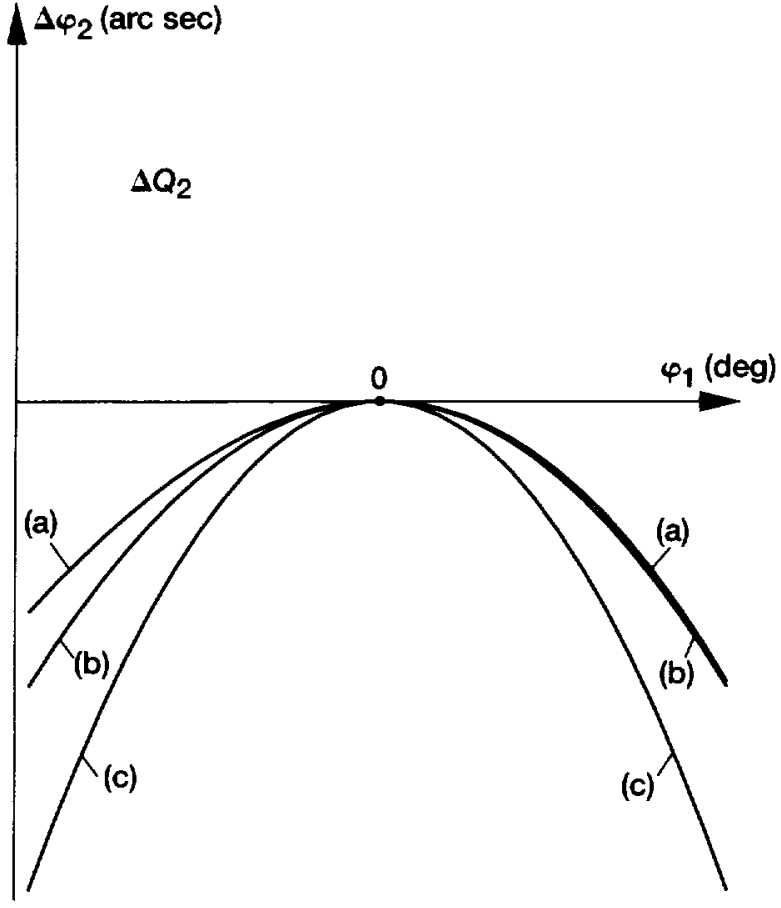

Figure 5.4.1.-Parabolic functions of transmission errors. (a) Obtained by simulation of meshing by TCA. (b) Obtained by approximate equation (5.4.3). (c) Obtained by approximate equation (5.4.12).

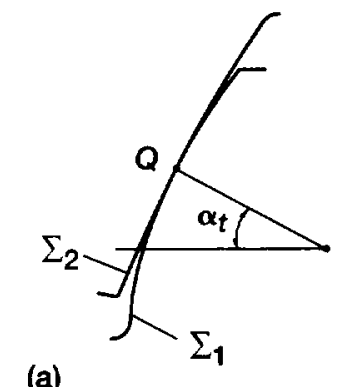

(a)

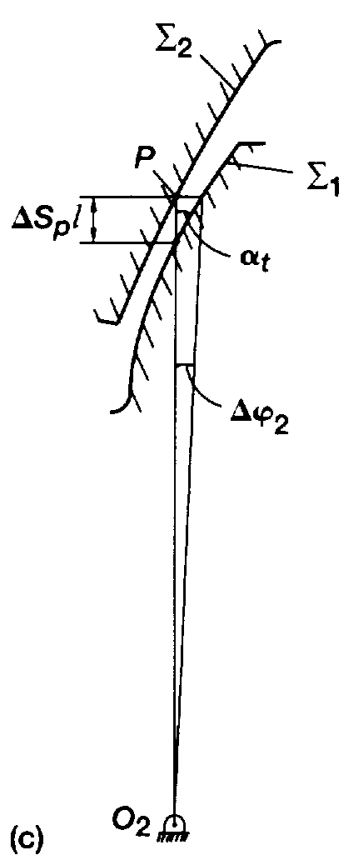

(b)

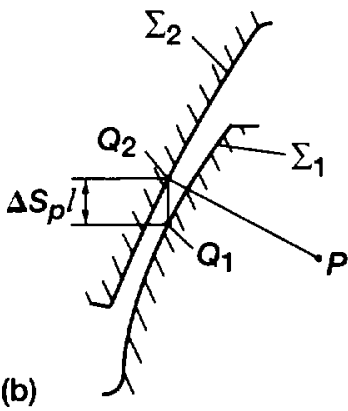

(c)

Figure 5.4.2.-Derivation of relation between coefficients of parabolas of transmission and plunging functions. (a) Transverse profiles $\Sigma_{1}$ and $\Sigma_{2}$ in initial meshing. (b) Transverse profiles after plunge of $\Sigma_{1}$. (c) Representation of $\Sigma_{1}$ and $\Sigma_{2}$ as equidistant profiles.

Step 2.-Figure 5.4.2(b) shows that due to the plunge of transverse profile $\Sigma_{1}$, there is a backlash between points $Q_{1}$ and $Q_{2}$ of the transverse profiles determined as

$$
\Delta s_{p l}=a_{p l}\left(\frac{B}{2}\right)^{2}
$$

Step 3. -For simplification, consider the equidistant transverse profiles (fig. 5.4.2(c)). The backlash $\Delta s_{p l}$ may be compensated after the turning of profile $\Sigma_{2}$ through angle

$$
\Delta \varphi_{2}=\frac{\Delta s_{p l} \tan \alpha_{t}}{r_{p 2}}
$$

where $\Delta \varphi_{2}$ is the transmission error.

The goal of plunging is to provide a parabolic function of transmission errors:

$$
\Delta \varphi_{2}\left(\varphi_{1}\right)=a_{\varphi} \varphi_{1}^{2}
$$

Step 4.-Figure 5.4.3 shows the development of the helix on the pinion pitch cylinder, where $H$ is the lead. Drawings of the figure yield

$$
B=\frac{r_{2} 2 \pi}{N_{1} \tan \beta}=\frac{\pi}{P_{f} \tan \beta}
$$




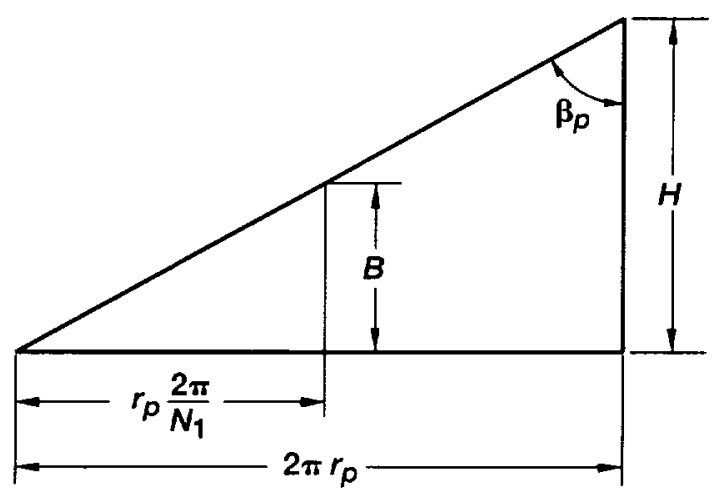

Figure 5.4.3.-Development of helix.

Step 5. -Equations (5.4.8) to (5.4.11), taking $\varphi_{1}=\pi / N_{1}$ in equation (5.4.1), yield the following relation between the coefficients of the parabolic functions of transmission and plunging:

$$
a_{\varphi}=\frac{N_{1}^{2} \tan \alpha_{t}}{2 P_{t} N_{2} \tan ^{2} \beta_{p}} a_{p l}=\frac{N_{1}^{2} \tan \alpha_{n}}{2 P_{n} N_{2} \sin ^{2} \beta_{p}} a_{p l}
$$

Function $\Delta \varphi_{2}\left(\varphi_{1}\right)$ determined by equation (5.4.12) is illustrated by curve $\mathrm{c}$ in figure 5.4.1.

For the case of a modified pinion ground by a shaped disk, the relations between the coefficients of the parabolic functions of transmission errors and plunging are similar to the relations represented by expressions (5.4.3) or (5.4.12). We have to accept only equivalent coefficients of plunging parabolas for grinding disk $a_{d}$ and for grinding worm $a_{p l}$ that are related by the following approximate equation:

$$
a_{d} \approx \frac{a_{p l} \sin \alpha_{t}}{\sin \left(\alpha_{t}+\varphi_{10}\right)}
$$

where $\varphi_{10}=\pi / N_{1}$.

\subsection{Tooth Contact Analysis}

The conditions of meshing and the contact of gears with the proposed geometry are investigated for an ideal and misaligned gear drive. The results of TCA are presented in the following two cases.

Case 1: pinion generated by grinding disk.-Figures 5.5.1 and 5.5.2 show the almost linear functions of transmission errors caused by errors $\Delta \gamma=3 \mathrm{arc} \min$ and $\Delta \lambda=3 \mathrm{arcmin}$. The magnitude of maximal transmission errors is $40.48 \mathrm{arc} \mathrm{sec}$ in both cases. Figure 5.5.3 shows the predesigned parabolic function for an aligned gear drive with the pinion generated by a grinding disk. The magnitude of the maximal transmission error is 11.54 arc sec. Figure 5.5.4 shows the resulting parabolic function of transmission errors when $\Delta \gamma=3 \mathrm{arc} \min$; the magnitude of the maximal transmission error is 11.66 arc sec. Figure 5.5.5 shows the resulting parabolic function of transmission errors when $\Delta \lambda=3 \mathrm{arc}$ min; the magnitude of the maximal transmission error is $10.11 \mathrm{arc} \mathrm{sec}$.

Case 2: pinion generated by worm.-Figures 5.5.6 and 5.5.7 show the almost linear functions of transmission errors caused by errors $\Delta \gamma=3 \mathrm{arc} \min$ and $\Delta \lambda=3 \mathrm{arc}$ min. The magnitude of maximal transmission errors is $40.48 \mathrm{arcsec}$ in both cases. Figure 5.5.8 shows the predesigned parabolic function for an aligned gear drive with the pinion generated by a worm. The magnitude of the maximal transmission error is 7.21 arc sec. Figure 5.5 .9 shows the resulting parabolic function of transmission errors when $\Delta \gamma=3 \mathrm{arc} \mathrm{min}$; the magnitude of the maximal transmission error is $8.33 \mathrm{arc} \mathrm{sec}$. Figure 5.5.10 shows the resulting parabolic function of transmission errors when $\Delta \lambda=3 \mathrm{arc}$ min; the magnitude of the maximal transmission error is $5.41 \mathrm{arc} \mathrm{sec}$. 


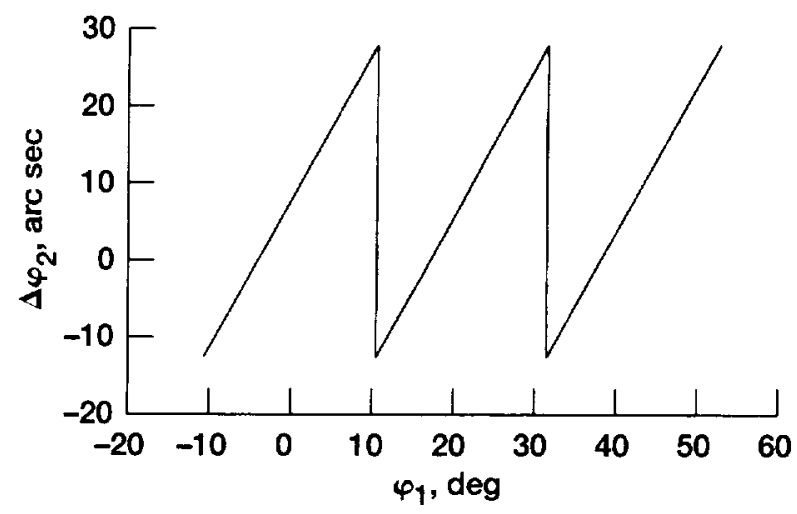

Figure 5.5.1.-Function of transmission errors of gear drive with pinion generated by grinding disk. Change in shaft angle $\Delta \gamma=3$ arc $\mathrm{min}$.

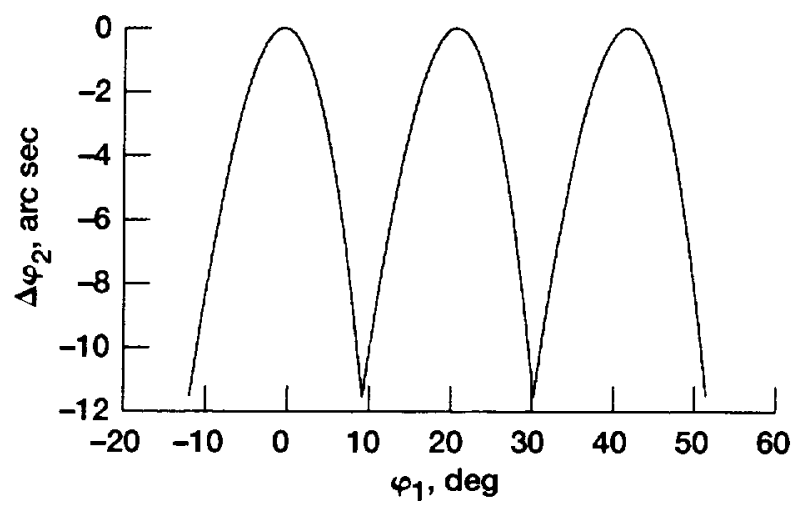

Figure 5.5.3.-Predesigned parabolic function for gear drive with pinion generated by grinding disk.

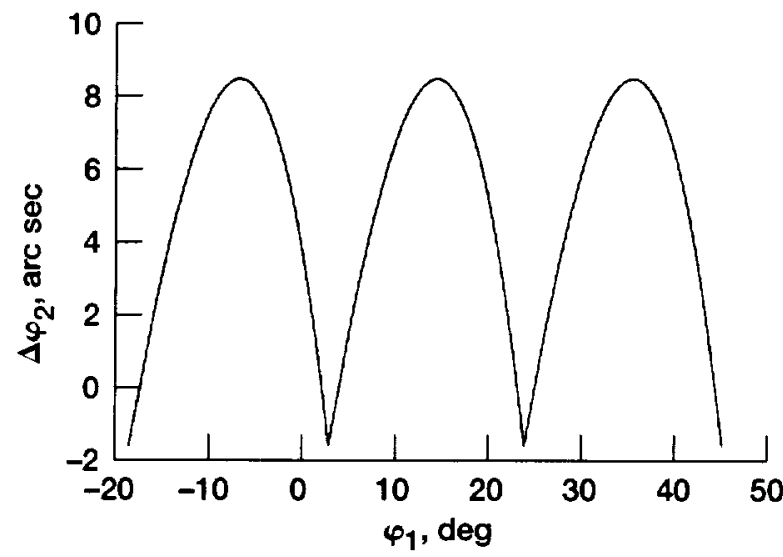

Figure 5.5.5.-Resulting function of transmission errors obtained for following conditions: pinion generated by a grinding disk; change in lead angle $\Delta \lambda=$ arc $\min$.

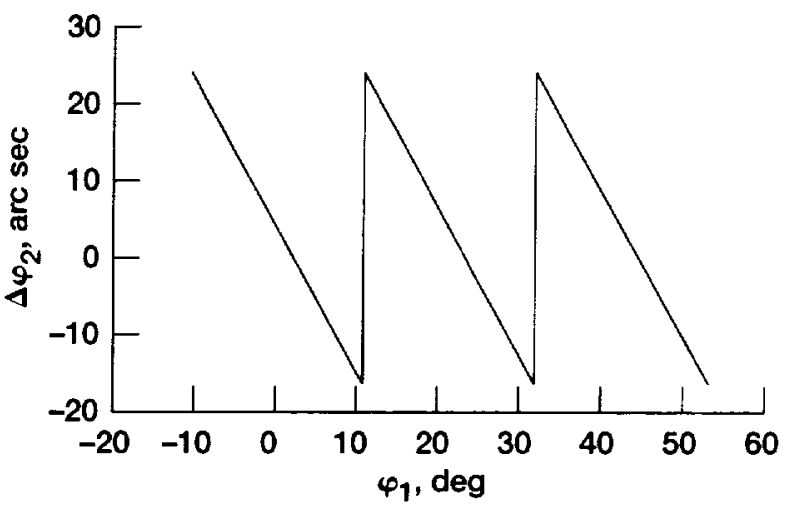

Figure 5.5.2.-Function of transmission errors of gear drive with pinion generated by grinding disk. Change in lead angle $\Delta \lambda=3$ arc min.

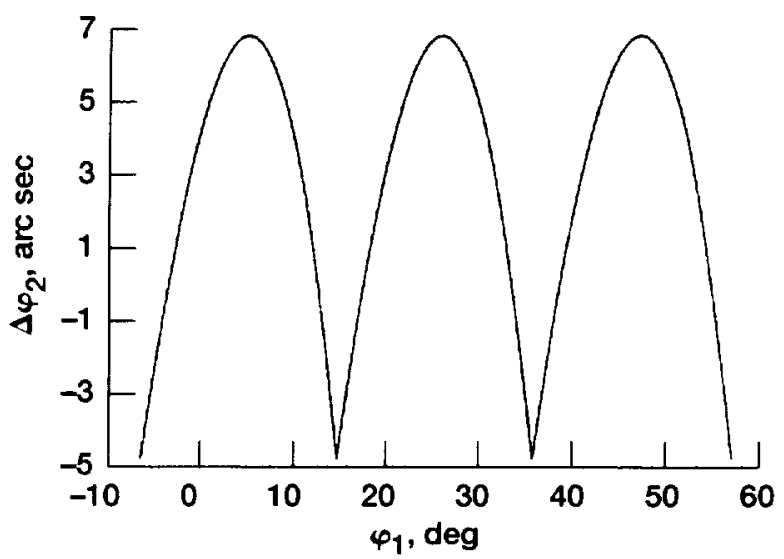

Figure 5.5.4.-Resulting function of transmission errors obtained for following conditions: pinion generated by a grinding disk; change in shaft angle $\Delta \gamma=$ arc $\mathrm{min}$.

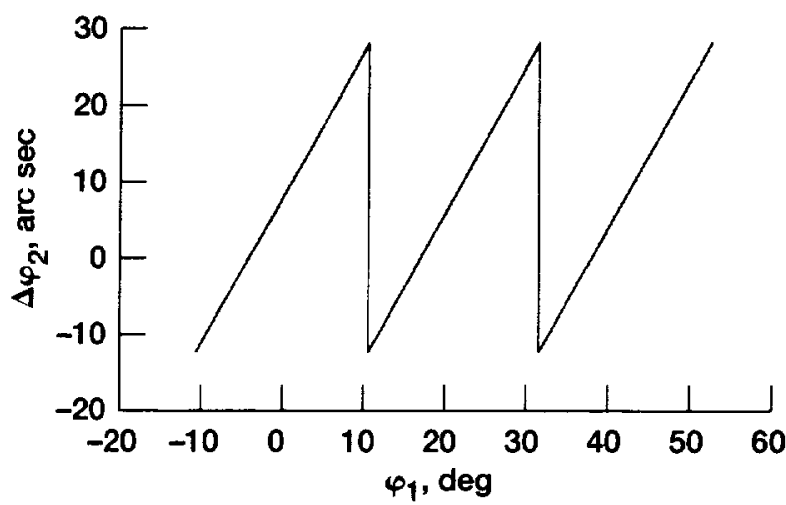

Figure 5.5.6.-Function of transmission errors of gear drive with pinion generated by worm; change in shaft angle $\Delta \gamma=3$ arc $\min$. 


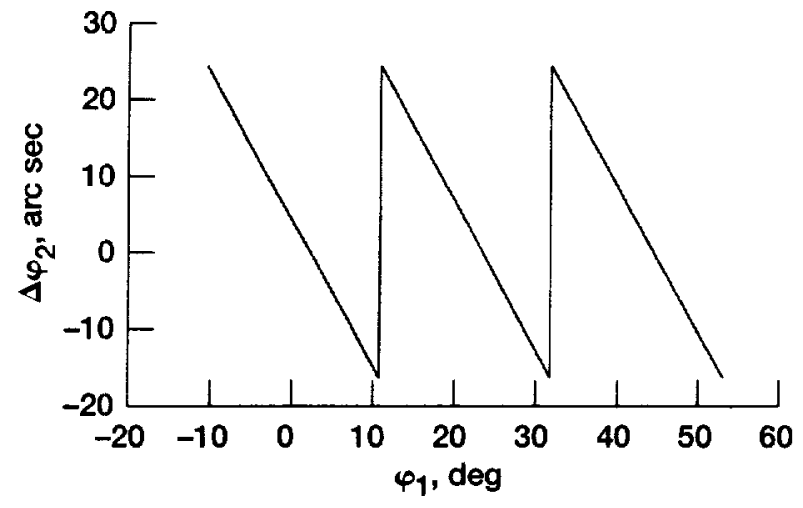

Figure 5.5.7.-Function of transmission errors of gear drive with pinion generated by worm; change in lead angle $\Delta y=3$ arc min.

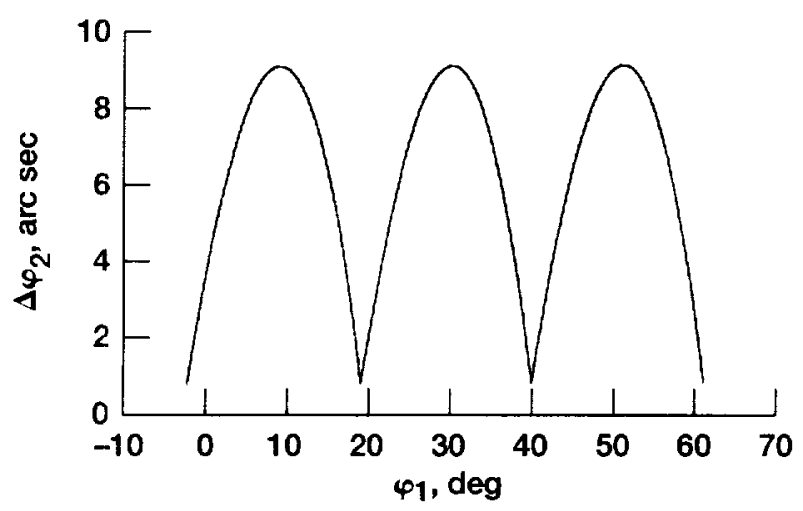

Figure 5.5.9.-Resulting function of transmission errors obtained for following conditions: pinion generated by worm; change in shaft angle $\Delta \gamma=\operatorname{arc} \min$.

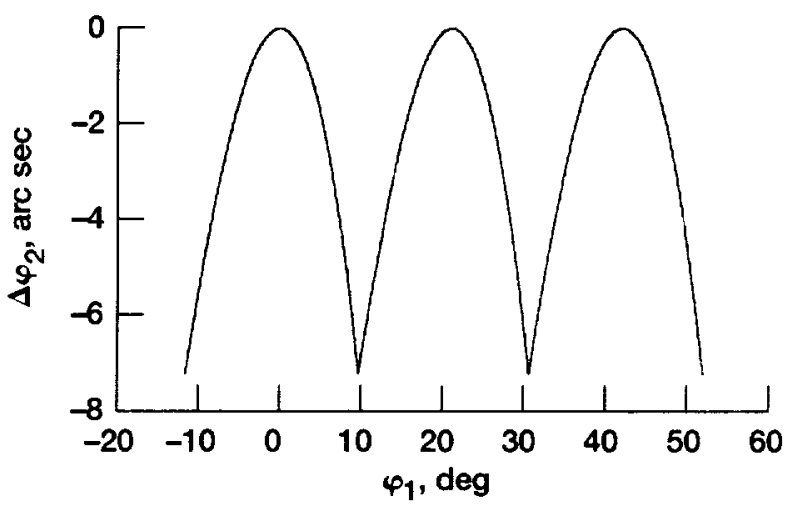

Figure 5.5.8.-Predesigned parabolic function for gear drive with pinion generated by worm.

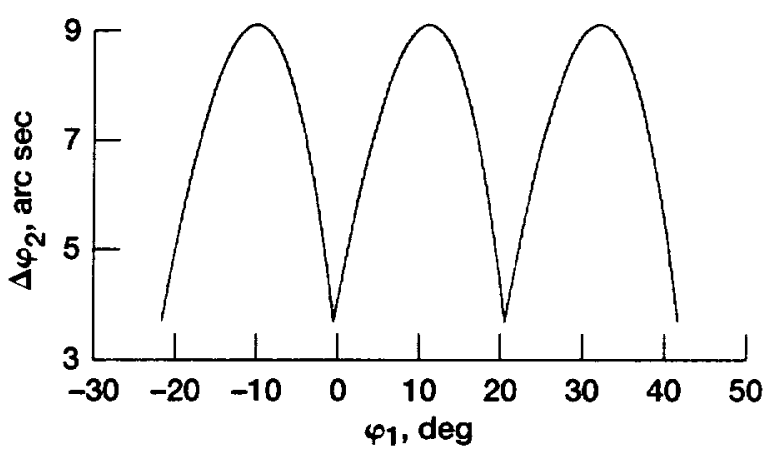

Figure 5.5.10.-Resulting function of transmission errors obtained for following conditions: pinion generated by worm; change in lead angle $\Delta \lambda=$ 3 arc $\mathrm{min}$.

Figure 5.5.11.-Contact ellipses on pinion tooth surface.

The completed computations have confirmed in both cases that the plunging of the tool provides a parabolic function of transmission errors and such a function absorbs transmission errors caused by misalignment. Figure 5.5.11 shows the contact ellipses on the pinion tooth surface. 


\section{APPENDIX C \\ DERIVATION OF GRINDING WORM THREAD SURFACE}

The generation of the worm thread surface $\Sigma_{n}$ is based on the following idea: The worm is a helicoid and is generated by an imaginary rack-cutter $\Sigma_{r}$, having the same normal section as the rack-cutter $\Sigma_{c}$ that is applied for the generation of the pinion.

The derivation of $\Sigma_{w}$ is based on this procedure:

Step 1.-Apply movable coordinate systems $S_{r}$ and $S_{n}$ that are rigidly connected to the imaginary rack-cutter and the worm. The fixed coordinate system $S_{n}$ is rigidly connected to the housing of the generating machine.

Step 2. - The rack-cutter $\Sigma_{r}$, has skew teeth determined by angle $\beta_{r}$ (fig. C.1), which is correlated with the worm lead angle $\lambda_{w}$. The rack-cutter surface is generated in $S_{r}$ while the coordinate system $S_{b}$ with the normal profile of the rack-cutter performs a translational motion along the direction of the skew rack-cutter teeth (along the $y_{b}$-axis).

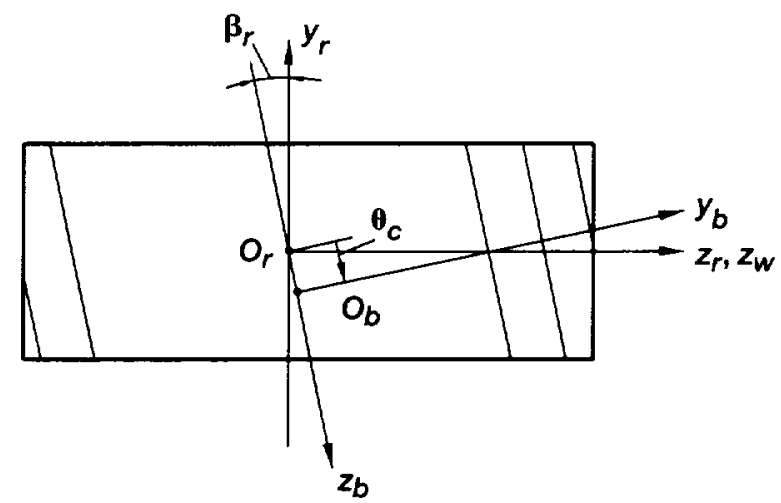

Figure C.1.-Skew rack-cutter $\sum_{r}$ used for worm generation.

Step 3.-Figure C.2 shows the installment of the left-hand worm with respect to rack-cutter $\Sigma_{r}$. It is obvious that axes $z_{b}$ and $z_{w}$, form the angle $90^{\circ}-\lambda_{w}$ and $\beta_{r}=\lambda_{w}$.

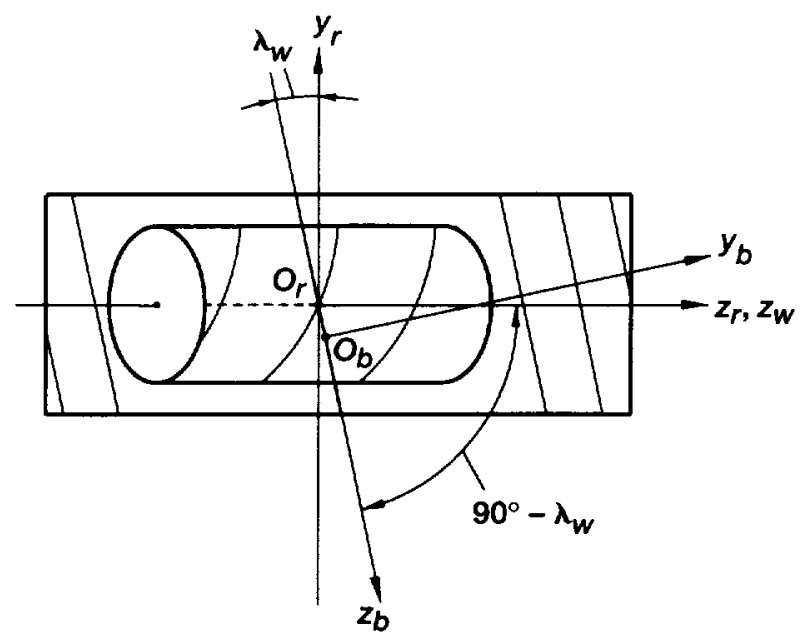

Figure C.2.-Installment of worm with respect to rack-cutter. 
Step 4.-During the worm generation process, the rack-cutter performs translation $S_{r}$ while the worm rotates through angle $\psi_{w^{\prime}}$ (fig. C.3), where

$$
s_{r}=r_{p w} \cdot \psi_{w}
$$

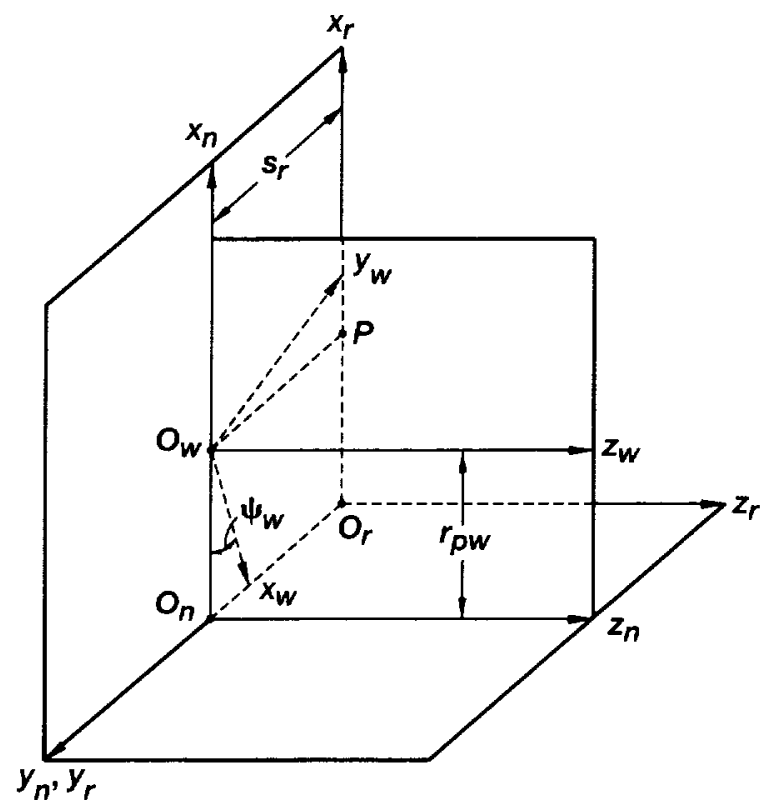

Figure C.3.-Applied coordinate systems for grinding worm and pinion.

Step 5. - The family of rack-cutter surfaces generated in $S_{\mathcal{W}}$ is represented by the matrix equation (fig. C.3)

$$
\mathbf{r}_{\mathrm{H}^{\prime}}\left(u_{c}, \boldsymbol{\theta}_{c}, \psi_{n^{\prime}}\right)=\mathbf{M}_{n^{\prime} n} \mathbf{M}_{n r} \mathbf{M}_{r b} \mathbf{r}_{b}\left(u_{c}\right)
$$

where (see eq. (2.2.2))

$$
\mathbf{r}_{b}\left(u_{c}\right)=\mathbf{M}_{b a} \mathbf{r}_{a}\left(u_{c}\right)
$$

Here

$$
\begin{gathered}
\mathbf{M}_{w n}\left(\psi_{w^{\prime}}\right)=\left[\begin{array}{cccc}
-\cos \psi_{w} & -\sin \psi_{w} & 0 & r_{p w^{\prime}} \cos \psi_{w} \\
\sin \Psi_{w} & -\cos \psi_{w} & 0 & -r_{p w} \sin \psi_{w} \\
0 & 0 & 1 & 0 \\
0 & 0 & 0 & 1
\end{array}\right] \\
\mathbf{M}_{n r}\left(\psi_{w}\right)=\left[\begin{array}{cccc}
1 & 0 & 0 & 0 \\
0 & 1 & 0 & -r_{p w} \psi_{w} \\
0 & 0 & 1 & 0 \\
0 & 0 & 0 & 1
\end{array}\right]
\end{gathered}
$$




$$
\mathbf{M}_{r b}\left(\theta_{c}\right)=\left[\begin{array}{cccc}
1 & 0 & 0 & 0 \\
0 & \sin \lambda_{w^{\prime}} & -\cos \lambda_{w} & -\theta_{c} \cos \lambda_{w^{\prime}} \\
0 & \cos \lambda_{w^{\prime}} & \sin \lambda_{w^{\prime}} & \theta_{c} \sin \lambda_{w^{\prime}} \\
0 & 0 & 0 & 1
\end{array}\right]
$$

Step 6. - The worm thread surface is the envelope to the family of surfaces $\mathbf{r}_{x^{\prime}}\left(u_{c}, \theta_{c}, \psi_{k}\right)$. To determine the envelope, apply the theorem (refs. 5 and 7) that the common normal to surfaces $\Sigma_{k}$ and $\Sigma_{r}$ passes through the instantaneous axis of rotation that coincides with the $=_{n}$-axis.

Then we obtain

$$
\frac{X_{r}-x_{r}}{N_{x r}}=\frac{Y_{r}-y_{r}}{N_{y r}}=\frac{Z_{r}-z_{r}}{N_{z r}}
$$

where (fig. C4) $X_{r}=0, Y_{r}=-s_{r}=-r_{p w} \psi_{u^{\prime}}, Z_{r}$ are the coordinates of a current point of the instantaneous axis of rotation; $\mathbf{N}_{r}$ is the normal to rack-cutter surface $\Sigma_{r}$ determined as

$$
\mathbf{N}_{r}=\mathbf{L}_{r b} \mathbf{L}_{b a} \mathbf{N}_{a}
$$

where (see fig. 2.1.2(b))

$$
\mathbf{N}_{a}=\left[\begin{array}{lll}
-2 a_{c} u_{c} & 1 & 0
\end{array}\right]^{T}
$$

The first equation of the system of equations (C7) yields

$$
x_{r}-\frac{\left(r_{\left.p_{w} \cdot \Psi_{w^{*}}+y_{r}\right) N_{x r}}\right.}{N_{y r}}=f\left(u_{c}, \theta_{c}, \psi_{w^{\prime}}\right)=0
$$

Equations $(\mathrm{C} 2)$ and $(\mathrm{Cl})$ represent the worm surface in three-parameter form. Equation (C10) is linear with respect to $\psi_{w}$, and $\theta_{c}$. Therefore $\theta_{c}$ can be represented by $\theta_{c}\left(u_{c}, \psi_{w}\right)$. Using this relation in equation (C2), the worm surface may be determined in two-parameter form as

$$
\mathbf{r}_{u^{\prime}}\left(u_{c}, \Psi_{w^{\prime}}\right)=\mathbf{M}_{u^{\prime} n}\left(\Psi_{w^{\prime}}\right) \mathbf{M}_{n r}\left(\Psi_{w^{\prime}}\right) \mathbf{M}_{r b}\left(\Psi_{w^{\prime}}\right) \mathbf{r}_{b}\left(u_{c}\right)
$$

\subsection{COMPARISON OF THREE TYPES OF NOVIKOV-WILDHABER HELICAL GEARS}

\subsection{Introduction}

The purpose of this section is to summarize the advantages and disadvantages of three types of NovikovWildhaber helical gears that will serve as directions for designers and manufacturers. For this purpose, section 6.2 will cover the following items:

1. Comparison of normal sections of rack-cutters used for generation

2. Description of existing and proposed methods for generation

3. Evaluation of the influence of errors of alignment on transmission errors and the shift of the bearing contact

4. Comparison of the dimensions of contact ellipses

5. Perspectives for the substitution of conventional involute helical gears by Novikov-Wildhaber helical gears 


\subsection{Description and Comparison of Three Types of Novikov-Wildhaber Gears}

Three versions of Novikov-Wildhaber gears are compared with (a) circular-arc profiles of rack-cutters, one zone of meshing, and a very small ( 0.5 percent) difference between the curvature radii of the rack-cutters (refs. 1 and 2 ); (b) circular-arc profiles of rack-cutters and two zones of meshing in accordance with Russian and Chinese standards (refs. 3 and 4); and (c) parabolic profiles of rack-cutters (proposed by the authors of this completed project).

Input data. - The results of a numerical computation performed for the three versions are based on the following input data:

Normal diametral pitch, $P_{n}$, in. $^{-1}$ 5

Pinion-gear teeth numbers

$$
N_{1} \text {......... }
$$

Shortest center distance, $E$, in

Pitch helix angle, $\beta$, deg

Normal pressure angle, $\alpha_{n}$, deg

Versions 1 and 2

Version 3

Profiles of rack-cutters.-The normal profiles of rack-cutters for three versions of the design are presented in figure 6.2.1.

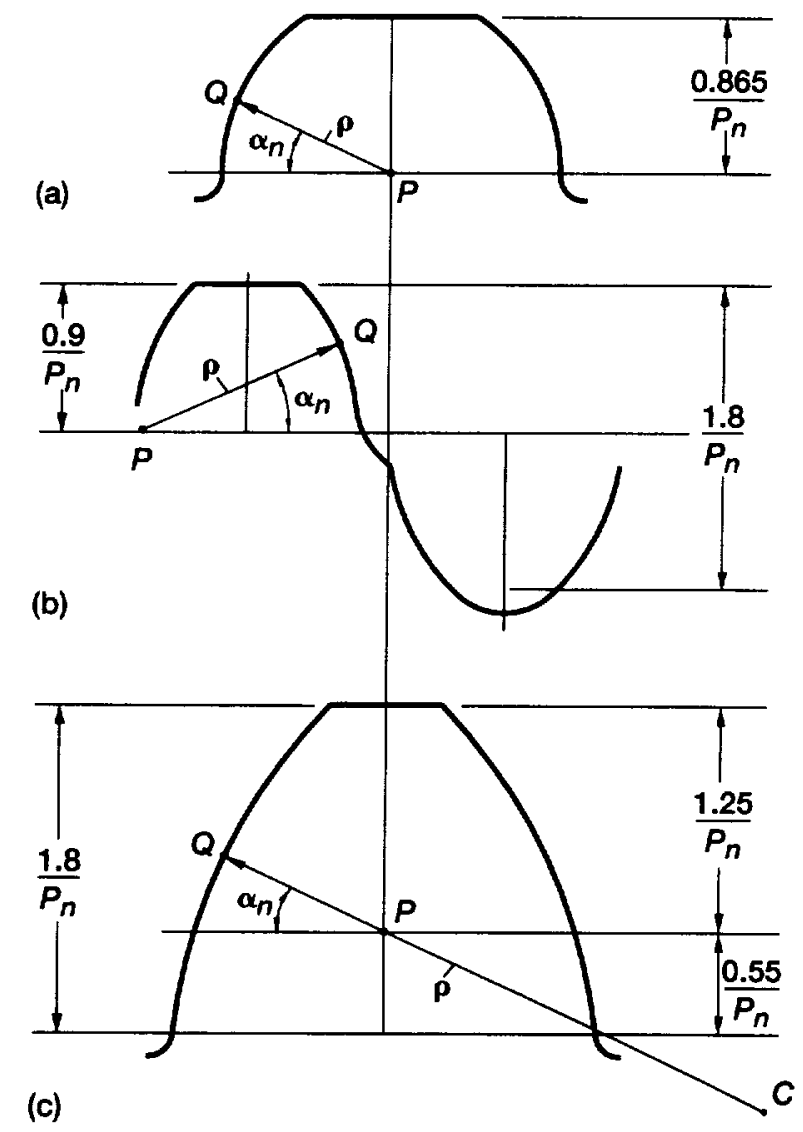

Figure 6.2.1.-Profiles of rack-cutters for generating pinion tooth. (a) Version 1. (b) Version 2. (c) Version 3. 
Versions 1 and 2 have circular-arc profiles. The centers of circular arcs that generate convex tooth addendums of the pinion (in both versions) and the gear (in version 2) are located on pitch straight lines of the rack-cutters.

In version 3 , the normal profiles are mismatched parabolic curves instead of circular arcs. In addition, the curvature radii of the parabolic curves are much larger than the distance $Q P$ between the designed point of tangency $Q$ and the pitch point $P$.

The normal curvature radii of the rack-cutters that generate the pinion and gear teeth are presented in table 6.2.1.

TABLE 6.2.1-NORMAL CURVATURE
RADII OF RACK-CUTTERS
\begin{tabular}{|c|c|c|}
\hline Version & $\begin{array}{c}\text { Pinion, } \\
\text { in. }\end{array}$ & $\begin{array}{c}\text { Gear, } \\
\text { in. }\end{array}$ \\
\hline 1 & 0.200 & 0.201 \\
2 & .230 & .258 \\
& $(.258)$ & $(.230)$ \\
3 & 1.176 & 1.270 \\
\hline
\end{tabular}

'For design 2, the upper (lower) values correspond to pinion addendum (dedendum) and gear dedendum (addendum)

Table 6.2.1 shows that design version 3 provides much larger curvature radii and therefore the tooth shape is more favorable.

The reader is reminded that the application of parabolic profiles of rack-cutters and the enlargement of the curvature radii allow one to increase the height of the pinion addendum, which is limited by tooth pointing, and the height of the pinion dedendum, which is limited by the possibility of undercutting.

The dimensionless heights of the active parts of the addendum $h_{a} P_{n}$ and the dedendum $h_{d} P_{n}$ of rack-cutters that generate the pinion teeth are shown in table 6.2.2, which gives data for three versions of the design.

TABLE 6.2.2.- RACK-CUTTER TOOTH
ELEMENT PROPORTIONS
\begin{tabular}{|c|c|c|c|}
\hline Version & \multicolumn{3}{|c|}{ Dimensionless heights of } \\
\cline { 2 - 4 } & $\begin{array}{c}\text { Addendum. } \\
h_{a} P_{n}\end{array}$ & $\begin{array}{c}\text { Dedendum, } \\
h_{d} P_{n}\end{array}$ & $\begin{array}{c}\text { Total } \\
\text { height, } \\
h P_{n}\end{array}$ \\
\hline 1 & 0.00 & 0.87 & 0.87 \\
2 & .90 & 90 & 1.80 \\
3 & .55 & 1.25 & 1.80 \\
\hline
\end{tabular}

Table 6.2.2 shows that the parabolic rack-cutter (design version 3 with one zone of meshing) has the same tooth height as that of the circular-arc rack-cutter (design version 2 with two zones of meshing):

$$
h=h_{a}+h_{d}=\frac{1.8}{P_{n}}
$$

Another important advantage of the developed gearing is the possibility of increasing the tooth thickness at the point of tangency of the active profile with the fillet. We may expect that this will allow designers to reduce the bending stresses, but this is a subject of further investigation. 
Another advantage of the developed gearing (design version 3 ) is the possibility of reducing the sensitivity of the gear drive to the change $\Delta E$ of the center distance. Table 6.2.3 presents for all three versions the influence of $\Delta E$ on the following parameters:

1. $\delta_{\alpha}:$ the normal pressure angle variation caused by the increase of $\Delta E=0.0001 \mathrm{~mm}$ of center distance $E$

2. $\delta_{1}$ : the ratio of the contact point shift over the pinion tooth profile to the tooth active height

Values $\delta_{\alpha}$ and $\delta_{1}$ have been determined twice by approximate formulas presented in section 2.0 and by the application of TCA in section 3.0.

The data of table 6.2 .3 show that

1. The original version 1 of Novikov-Wildhaber gears is not applicable in a real design because of a high sensitivity to the change of center distance.

2. Version 3 is less sensitive to error $\Delta E$ in comparison with version 2 and therefore is preferable.

TABLE 6.2.3-SENSITIVITY TO CHANGE

OF THE CENTER DISTANCE $\triangle E$

\begin{tabular}{|c|c|c|}
\hline Version & $\begin{array}{c}\delta_{\mathrm{a}}, \\
\mathrm{deg}\end{array}$ & $\boldsymbol{\delta}_{l}$ \\
\hline 1 & 19.50 & 0.383 \\
2 & 2.28 & 0.051 \\
3 & 0.68 & 0.038 \\
\hline
\end{tabular}

Contact ellipses and their orientation.-The contact ellipses and their orientation with respect to the tangent of the contact path (determined by angle $\mu_{1}$ ) are shown in figure 6.2.2. The parameters of the contact ellipse axes for three versions of the design are presented in table 6.2.4.

TABLE 6.2.4.-PARAMETERS OF CONTACT ELLIPSE

[All values are determined for elastic approach, $\delta=0.00025$ in.]

\begin{tabular}{|c|c|c|c|}
\hline Version & $\begin{array}{c}\text { Major axis, } \\
2 a, \\
\text { in. }\end{array}$ & $\begin{array}{c}\text { Minor axis, } \\
2 b, \\
\text { in. }\end{array}$ & $\begin{array}{c}\text { Area, } \\
F, \\
\text { in. }\end{array}$ \\
\hline 1 & 0.285 & 0.237 & 0.053 \\
2 & .237 & .065 & .012 \\
3 & 1.034 & .045 & .037 \\
\hline
\end{tabular}

Figure 6.2.2. and table 6.2.4 show that "ideal" version 1 has the most favorable contact ellipse parameters. However, as mentioned in the previous paragraphs, this version cannot be applied in practice because of the high sensitivity to the change $\Delta E$ of the center distance. This defect requires that the difference of curvature radii be increased as was foreseen in version 2 . Therefore, the area of the contact ellipse in version 1 is decreased by more than four times.

The proposed design version 3 has a contact ellipse area three times larger than the standard version 2 . We may expect that this will substantially decrease the contact stresses. Therefore, we consider that version 3 has a great advantage in comparison with the existing Novikov-Wildhaber gear designs. 

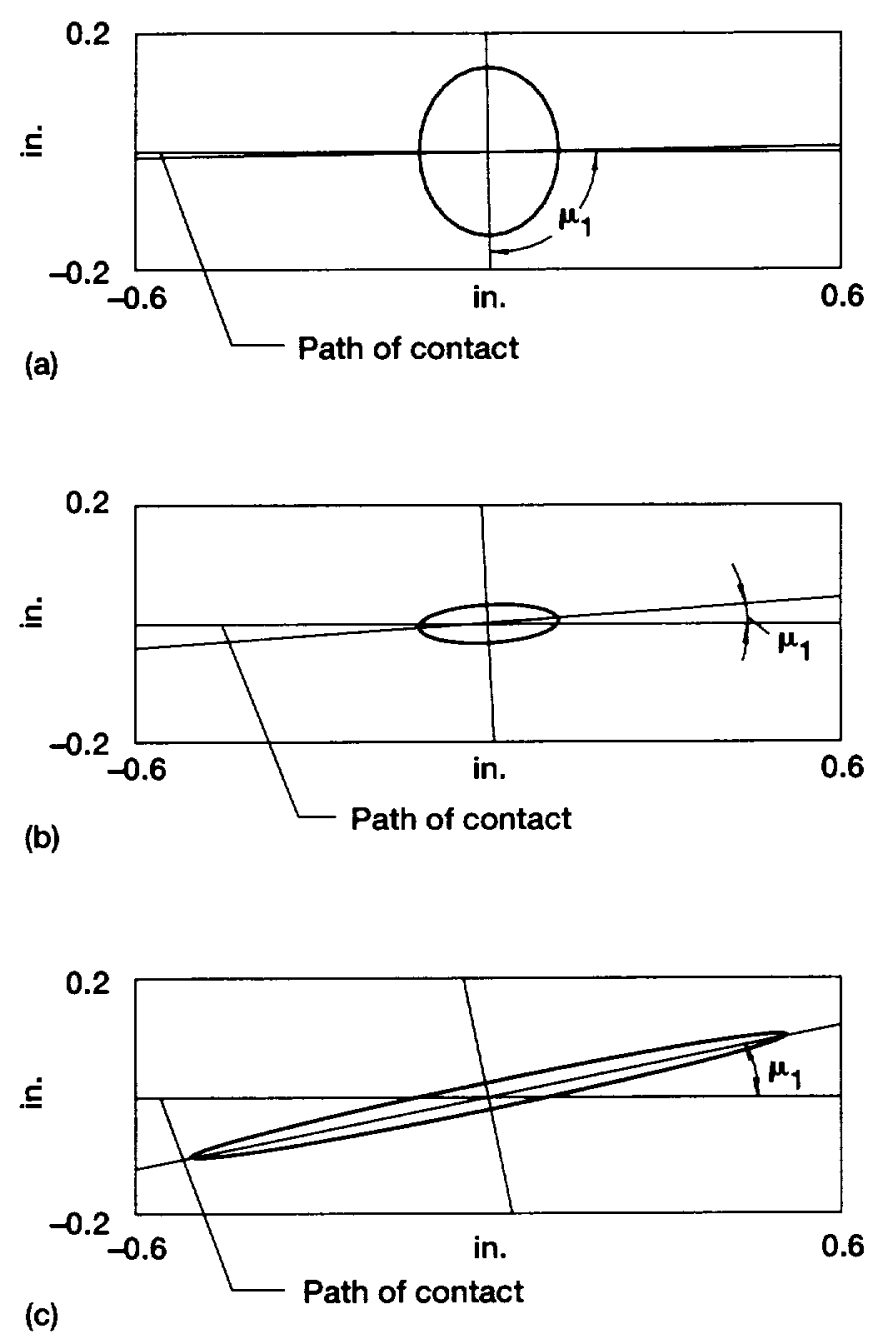

Figure 6.2.2.-Contact ellipses on pinion tooth surface.

(a) Version 1. (b) Version 2. (c) Version 3.

\subsection{Methods of Generation in Industrial Application}

Gears of designs 1 and 2 can be manufactured only by hobbing. Helical gears of the new design (version 3 ) can be ground as proposed by a shaped grinding disk or by a grinding worm.

Presently, gears of designs 1 and 2 are applied to avoid the surface hardening that follows grinding. A good bearing contact for such versions of the design is achieved by running the gear drives in the housing. Therefore, helical gear designs 1 and 2 cannot compete with widely used conventional involute helical gears for which surface hardening is achieved by carbonizing and nitriding after grinding. Thus, Novikov-Wildhaber gears are not applied yet in high-power and high-speed reducers. The new type of helical gears (design 3) developed in this project is free of the disadvantages of existing Novikov-Wildhaber gears since the gear tooth surfaces can be hardened and ground.

The greatest disadvantage of design versions 1 and 2 is that only profile crowning of tooth surfaces is provided. This means that in these two cases only localization of bearing contact can be achieved, but the gear drives are very sensitive to misalignment and noise. The gear drive vibration is inevitable.

The newly developed gearing (design 3 ) is free of the disadvantages mentioned in the previous paragraph because the design and manufacture of the gear drives are based on a combination of profile and longitudinal crowning. It was shown in sections 4.0 and 5.0 that such crowning makes it possible to obtain a favorable shape of transmission error function, reduce the level of transmission errors, and as a result, reduce the noise and vibration. 


\subsection{CONCLUSION}

A new geometry for Novikov-Wildhaber helical gears was developed, and contact stresses were reduced as a result of the contact of convex-concave gear tooth surfaces.

The conjugation of gear tooth surfaces with profile crowning was achieved by applying two rack-cutters with parabolic profiles in the normal section. In addition, crowning in the longitudinal direction provides a predesigned parabolic function of transmission errors. This type of transmission error enables the design to absorb linear function errors caused by misalignment and to reduce the noise and vibration.

Methods of generating tooth surfaces by worm grinding, form grinding, and hobbing were developed.

Computerized simulation of meshing and contact was performed and a respective computer program for the investigation of transmission errors and the shift of the bearing contact was also developed. The output of the computer program confirmed the accuracy of the proposed approaches reported herein.

\subsection{REFERENCES}

1. Wildhaber, E.: Helical Gearing, U.S. Patent No. 1,601,750, 1926.

2. Novikov, M.L.: U.S.S.R. Patent No. 109,750, 1956.

3. U.S.S.R. Standard: GOST 15203-76, 1976.

4. Chinese Standard: J.B. 2940-81, 1981.

5. Litvin, F.L.: Theory of Gearing, NASA RP-1212 (AVSCOM 88-C-035), 1989.

6. Litvin, F.L., et al.: Computerized Design and Generation of Low-Noise Helical Gears With Modified Surface Topology. J. Mech. Des., ASME trans., vol. 117, no. 2, June 1995, pp. 254-261.

7. Litvin, F.L.: Gear Geometry and Applied Theory. Prentice-Hall, Englewood Cliffs, NJ, 1994.

8. Litvin, F.L.; and Lu, J.: Computerized Design and Generation of Circular-Arc Helical Gears with Low Transmission Errors. Comp. Methods Appl. Mech. Eng., vol. 127, no. 1-4, Nov. 1995, pp. 57-86.

9. IMSL MATH/LIBRARY: User's Manual, MALB-USM-UNBND-EN8901-1.1, 2500, Houston, TX, 1989. 

Public reporting burden for this collection of information is estimated to average 1 hour per response, including the time for reviewing instructions, searching existing data sources, gathering and maintaining the data needed, and completing and reviewing the collection of intormation. Send comments regarding this burden estimate or any other aspect of this collection of information, including suggestions for reducing this burden, to Washington Headquarters Services, Directorate for Intormation Operations and Reports, 1215 Jefferson Davis Highway, Suite 1204, Arlington, VA 22202-4302, and to the Office of Management and Budget, Paperwork Feduction Project (0704-0188), Washington, DC 20503.

\begin{tabular}{|l|l|l|}
\hline 1. AGENCY USE ONLY (Leave blank) & $\begin{array}{c}\text { 2. REPORT DATE } \\
\text { October } 2000\end{array}$ & $\begin{array}{c}\text { 3. REPORT TYPE AND DATES COVERED } \\
\text { Final Contractor Report }\end{array}$
\end{tabular}

\section{TITLE AND SUBTITLE}

Computerized Generation and Simulation of Meshing and Contact of

New Type of Novikov-Wildhaber Helical Gears

6. AUTHOR(S)

Faydor L. Litvin, Pin-Hao Feng, and Sergei A. Lagutin

7. PERFORMING ORGANIZATION NAME(S) AND ADDRESS(ES)

The University of Illinois at Chicago

Chicago, Illinois 60607

\section{FUNDING NUMBERS}

WU-581-30-13-00

$$
\text { NAG3-1992 }
$$

IL162211A47A

8. PERFORMING ORGANIZATION REPORT NUMBER

$$
\text { E-12039 }
$$

9. SPONSORINGMONITORING AGENCY NAME(S) AND ADDRESS(ES)

National Aeronautics and Space Administration

Washington, DC 20546-0001

and

U.S. Army Research Laboratory

Adelphi. Maryland 20783-1145
10. SPONSORINGMONITORING AGENCY REPORT NUMBER

NASA CR-2000-209415

ARL-CR-428

11. SUPPLEMENTARY NOTES

Project Manager, Robert Handschuh, U.S. Army Research Laboratory, Structures and Acoustics Division,

NASA Glenn Research Center, organization code 5950 (0300), 216-433-3969.

12a. DISTRIBUTION/AVAILABILITY STATEMENT

12b. DISTRIBUTION CODE

Unclassified - Unlimited

Subject Category: 37

Distribution: Nonstandard

Available electronically at http://gltrs.gac,nasa.gov/GLTRS

This publication is available from the NASA Center for AeroSpace Information. (301) $621-0390$.

13. ABSTRACT (Maximum 200 words)

In this report, we propose a new geometry for low-noise, increased-strength helical gears of the Novikov-Wildhaber type. Contact stresses are reduced as a result of their convex-concave gear tooth surfaces. The gear tooth surfaces are crowned in the profile direction to localize bearing contact and in the longitudinal direction to obtain a parabolic function of transmission errors. Such a function results in the reduction of noise and vibrations. Methods for the generation of the proposed gear tooth surfaces by grinding and hobbing are considered, and a tooth contact analysis (TCA) computer program to simulate meshing and contact is applied. The report also investigates the influence of misalignment on transmission errors and shift of bearing contact. Numerical examples to illustrate the developed approaches are proposed. The proposed geometry was patented by Ford/UIC (Serial Number 09-340-824, pending) on June 28, 1999.

\section{SUBJECT TERMS}

15. NUMBER OF PAGES 55

Geometry; Gears; Helical; Novikov; Wildhaber

17. SECURITY CLASSIFICATION OF REPORT

Unclassified

18. SECURITY CLASSIFICATION OF THIS PAGE

Unclassified
19. SECURITY CLASSIFICATION OF ABSTRACT Unclassified
$\mathrm{A} 04$

20. LIMITATION OF ABSTRACT

